\title{
DIFFUSION IN ENERGY CONSERVING COUPLED MAPS
}

\author{
JEAN BRICMONT AND ANTTI KUPIAINEN
}

\begin{abstract}
We consider a dynamical system consisting of subsystems indexed by a lattice. Each subsystem has one conserved degree of freedom ("energy") the rest being uniformly hyperbolic. The subsystems are weakly coupled together so that the sum of the subsystem energies remains conserved. We prove that the subsystem energies satisfy the diffusion equation in a suitable scaling limit.
\end{abstract}

\section{Coupled Maps with a Conservation Law}

1.1. Diffusion in Hamiltonian Dynamics. One of the fundamental problems in deterministic dynamics is to understand the microscopic origin of diffusion. On a microscopic level, a physical system such as a fluid or a crystal can be modeled by Schrödinger or Hamiltonian dynamics, with a macroscopic number of degrees of freedom. Although the microscopic dynamics is not dissipative, dissipation should emerge in large spatial and temporal scales e.g. in the form of diffusion of heat or of concentration of particles.

Dynamically, diffusion is related to the existence in the system of conserved quantities such as the energy which are extensive i.e. sums (or integrals) of local contributions that are "almost conserved". Thus, if the system has a microscopic energy density $E(t, x), x \in \mathbb{R}^{d}$ the total energy $E_{\text {tot }}=\int E(t, x) d x$ is a constant of motion but the energy density is, in general, not conserved since the dynamics redistributes it:

$$
\dot{E}(t, x)=\nabla \cdot \mathbf{J}(\mathbf{t}, \mathbf{x})
$$

The divergence acting on the energy current $J$ guarantees conservation of the total energy. One would like to show that the conservative dynamics (1.1) turns, in a suitable scaling limit, to a diffusive one. Such a limit involves diffusive scaling of space and time, and taking typical initial conditions with respect to the Liouville measure with prescribed initial energy profile. The resulting macroscopic energy density should then satisfy a nonlinear diffusion equation of the type

$$
\partial_{t} E=\nabla \cdot(\kappa(E) \nabla E)
$$

where $\kappa(E)$ is the conductivity function.

There has been a lot of numerical and theoretical work in recent years around these questions in the context of coupled dynamics. One considers a dynamical system consisting of a large number of elementary systems indexed by a subset $V$ of a $d$ dimensional lattice $\mathbb{Z}^{d}$. The total energy $E$ of the system is a sum $\sum_{x \in V} E(x)$ of energies $E(x)$ which involve the dynamical variables of the system at lattice site $x$ and

Date: 28.10 .10 .

Partially supported by the Belgian IAP program P6/02.

Supported by the Academy of Finland and European Research Council. 
nearby sites and describe the energy of the system at $x$ and its interaction energy with its neighbors.

In particular, two classes of models have been discussed. The first consists of Hamiltonian dynamics of coupled weakly anharmonic oscillators. In the weak anharmonicity scaling limit, one may use kinetic theory to compute the conductivity $\kappa$. Rigorous justification of the kinetic limit (see [22]), let alone the case of fixed (small) coefficient of the anharmonic term (see [8]), is still lacking.

A second class of models deals with a complementary situation of weakly coupled chaotic systems. Hamiltonian systems of this type are obtained by putting at each lattice site a chaotic system, e.g. a billiard, and coupling them weakly to each other [12. On might hope that the strong mixing properties of the billiard dynamics could help in proving diffusion. Rigorous results on such Hamiltonian systems are rare: in [12] ergodicity is proved for the one dimensional case. Numerically, diffusion comes out cleanly [19].

In this paper we will consider these issues in the framework of Coupled Map Lattices (CML) which are discrete time models for extended dynamical systems. We introduce a class of Coupled Map Lattices which satisfy a discrete space and time version of the conservation law (1.1). These systems have, apart from the conserved "energy" variables, chaotic variables that are coupled to the energies. We formulate for such systems a general framework for the derivation of the diffusive dynamics (1.2) and prove diffusion for a class of such CML's.

Our approach is to view the fast chaotic dynamics as a noise acting on the slow dynamics. We show that under quite general assumptions the slow dynamics becomes nonlinear diffusion in a random environment. We then prove diffusive scaling limit under the assumption of weak nonlinearity and weak randomness. The proof is based on a multi scale Renormalization Group (RG) method. In RG jargon, we show that both the noise and the nonlinearity are irrelevant. We want to stress that the systems we consider are far from realistic Hamiltonian systems like the ones mentioned above. The most radical simplification we make is to assume that the slow dynamics cannot slow down the fast one. This will not hold in Hamiltonian systems. However, such slowing down can in principle be studied using the RG and we view the paper as a first step in such studies.

1.2. Coupled Map Lattices. A CML is a dynamical system built out of subsystems $\left(M_{x}, f_{x}\right)$ indexed by the lattice $\mathbb{Z}^{d}$. The dynamical systems $f_{x}: M_{x} \rightarrow M_{x}$ are copies of a fixed system $(M, f)$. Let $\Lambda \subset \mathbb{Z}^{d}$. The phase space of the CML in volume $\Lambda$ is $\mathcal{M}_{\Lambda}=\times_{x \in \Lambda} M_{x}$ and the CML dynamics $\mathcal{F}: \mathcal{M}_{\Lambda} \rightarrow \mathcal{M}_{\Lambda}$ is a perturbation of the uncoupled dynamics $\times_{x \in \Lambda} f_{x}$.

To motivate our choice of $M$ and $\mathcal{F}$ consider again the coupled billiard case. The billiard dynamics has very good chaotic properties. There are two zero Lyapunov exponents corresponding to the conserved energy and the time shift ( $\nabla H$ and the Hamiltonian vector field). A discrete time version (given, say, by a Poincare map) would have one vanishing exponent and the remaining ones nonzero (positive and negative).

The following general class of CML models this situation. We let the subsystems have $M=\mathbb{R}_{+} \times N$ and denote the elements of $M$ by $(E, \theta)$. Hence at lattice site $x \in \mathbb{Z}^{d}$ 
we have $(E(x), \theta(x))$. We call energy the non-negative variables $E(x)$. $\theta$ represent the chaotic variables in the billiard case and they will take values in some manifold $N$.

The uncoupled dynamics is simply defined to be

$$
(E(x), \theta(x)) \rightarrow(E(x), g(\theta(x), E(x)))
$$

for each $x \in \mathbb{Z}^{d}$. The energies at each lattice site are thus conserved (as in the Hamiltonian case). The variables $\theta(x)$ are the fast, chaotic ones. In the billiard case, the dynamical system $\theta \rightarrow g(\theta, E)$ is uniformly hyperbolic for any fixed E. We will model this situation by taking $g(\theta, E)=g(\theta)$ a fixed chaotic map, independent of $E$. Examples are given by $N=\mathbb{T}^{1}=\mathbb{R} / \mathbb{Z}$ and $g$ an expansive circle map, e.g. $g(\theta)=2 \theta$ or $N=\mathbb{T}^{2}=\mathbb{R}^{2} / \mathbb{Z}^{2}$ and $g$ a hyperbolic toral automorphism. In general we will need good chaotic properties of the $\theta$ dynamics, in particular an invariant Sinai-Ruelle-Bowen (SRB) measure of Gibbsian type (see below).

We want to stress that in a realistic Hamiltonian system, such as the billiard, the $E$ dependence of $g$ can not be ignored. Indeed, it is obvious that, as $E \rightarrow 0$, the Lyapunov exponents of $g(\cdot, E)$ also tend to zero since $E$ sets the time scale.

1.3. Conservative Coupling. The CML dynamics is now written as

$$
\mathcal{F}(x, E, \theta)=(E(x)+\phi(x, E, \theta), g(\theta(x))+\psi(x, \theta))
$$

where $\phi$ and $\psi$ are the perturbation of the local dynamics and will be taken to be small local functions of $(E, \theta)$ (for $\phi$ ) or of $\theta$ (for $\psi$ ) i.e. to depend weakly on $(E(y), \theta(y))$ for $|x-y|$ large.

We will consider perturbations such that the total energy

$$
\sum_{x} E(x)
$$

is conserved. This follows if, formally,

$$
\sum_{x} \phi(x, E, \theta)=0
$$

for all $E, \theta$. A natural way to guarantee this is to consider a "vector field"

$$
\mathbf{J}(x)=\left\{J^{\mu}(x)\right\}_{\mu=1, \ldots, d}
$$

and take

$$
\phi(x, E, \theta)=(\nabla \cdot \mathbf{J})(x, E, \theta):=\sum_{\mu}\left(J^{\mu}\left(x+e_{\mu}, E, \theta\right)-J^{\mu}(x, E, \theta)\right),
$$

where $\left(e_{\mu}\right)_{\mu=1}^{d}$ is the canonical basis in $\mathbb{Z}^{d}$. With these definitions, we arrive at the following class of dynamical systems which are natural discrete space time versions of (1.1):

$$
\begin{aligned}
E(t+1, x) & =E(t, x)+\nabla \cdot \mathbf{J}(x, E(t), \theta(t)):=F(x, E(t), \theta(t)) \\
\theta(t+1, x) & =g(\theta(t, x))+\psi(x, \theta(t)):=h(x, \theta(t)) .
\end{aligned}
$$

Examples of the dynamics (1.6) will be discussed in Remark 2.4 below.

The purpose of this paper is to prove that the deterministic, conservative dynamical system (1.6), (1.7) gives rise to diffusion under suitable assumptions on the functions $\mathbf{J}$ and $h$. 


\section{The Result}

2.1. Random Environment. We would like to inquire under what conditions and in what sense the dynamics (1.6) of the energies is diffusive. Let us start by specifying in what sense we want to prove diffusion. The strongest and most natural result would be to prove diffusion for almost all initial values of $\theta$. By "almost all" we mean the following.

Suppose first $\psi=0$. The $\theta$ dynamics is then local and the $g$ dynamics has an invariant Sinai-Ruelle-Bowen measure $\nu_{0}$ on $N$. In this case, it would be natural to prove that the $E$-dynamics is diffusive (in a sense to be specified below) a.s. in $\theta(0, \cdot)$ with respect to the measure $\nu=\nu_{0}^{\mathbb{Z}^{d}}$.

For $\psi \neq 0$, if the $\theta$-dependence of $\psi$ is local and smooth and $\psi$ small in a suitable sense [11, 20, 21, 25, 3, 1, 2, 6, 7] the $\theta$ dynamics still has an invariant SRB measure $\nu$ defined on the cylinder sets of $N^{\mathbb{Z}^{d}}$. Then we want to prove diffusion a.s. with respect to $\nu$.

Sampling $\theta(0, \cdot)$ with the probability measure $\nu$ makes $\theta(t, x)$ random variables on the same probability space. Thus the $\theta$ dynamics provides a space time random environment for the E-dynamics:

$$
\mathbf{J}_{t}(x, E):=\mathbf{J}(x, E, \theta(t))
$$

are random functions of $E$. For $h$ as above, the $\theta$ dynamics is exponentially mixing in time and space. If the $\theta$-dependence of $\mathbf{J}$ is suitably local, then $\mathbf{J}_{t}(x, E)$ are weakly correlated random variables. We will next spell out these facts precisely.

2.2. Gibbs states. We assume $g$ is a uniformly expansive (i.e. $\left|g^{\prime}\right| \geq \lambda>1$ ) $C^{1+\alpha}$ circle map or a $C^{1+\alpha}$ perturbation of a linear toral hyperbolic automorphism. For the coupling assume $\psi$ is $C^{2}$ (actually $C^{1+\alpha}$ is enough, see [7]) and local, namely

$$
\left\|\frac{\partial^{2} \psi(x, \theta)}{\partial \theta_{y} \partial \theta_{z}}\right\|_{\infty} \leq \kappa e^{-m(|x-y|+|x-z|)}
$$

for some $m>0$ and $\kappa$ small enough. Here $\|f\|_{\infty}=\sup _{\theta \in N^{d}}|f(\theta)|$.

Then the following hold (see Appendix):

First, there is a map $\Gamma: \Omega \rightarrow N^{\mathbb{Z}^{d}}$ where

$$
\Omega=\times_{(x, t) \in \mathbb{Z}^{d+1}} \Omega_{x, t}
$$

(or a subset thereof, see Appendix) where $\Omega_{x, t}$ is a copy of a given finite set (whose elements will be called "spins") such that $\Gamma$ conjugates the dynamical system $\left(N^{\mathbb{Z}^{d}}, h\right)$ in (1.7) to a time shift $\tau$ in a space of symbol sequences

$$
h \circ \Gamma=\Gamma \circ \tau .
$$

Secondly $\left(N^{\mathbb{Z}^{d}}, h\right)$ has an invariant SRB measure $\nu$ which is the image under $\Gamma$ of a Gibbs measure $\mu$ on the $\sigma$-algebra $\Sigma$ generated by the cylinder sets of $\Omega$ :

$$
\int F(\theta) \nu(d \theta)=\int(F \circ \Gamma)(\omega) \mu(d \omega) .
$$

This Gibbs state is local in the following sense. For $A \subset \mathbb{Z}^{d+1}$ a finite set let $\Omega_{A}=$ $\times_{(x, t) \in A} \Omega_{x, t}$. We say $F: \Omega_{A} \rightarrow \mathbb{R}$ is supported in $A$. Then 
(a) There exists $m>0, C<\infty$, such that, for all $F_{1}, F_{2}$, with $F_{i}$ supported in $A_{i}$

$$
\left|\mathbb{E}\left(F_{1} F_{2}\right)-\mathbb{E}\left(F_{1}\right) \mathbb{E}\left(F_{2}\right)\right| \leq C \min \left(\left|A_{1}\right|,\left|A_{2}\right|\right)\left\|F_{1}\right\|_{\infty}\left\|F_{2}\right\|_{\infty} e^{-m d\left(A_{1}, A_{2}\right)},
$$

where $d\left(A_{1}, A_{2}\right)$ is the distance between the sets $A_{1}$ and $A_{2}$ and $\mathbb{E} F=\int F d \mu$.

(b) There exists $m>0, C<\infty$ such that, if $F_{i} \geq 0$ are supported on $A_{i}, i=1, \ldots, k$

$$
\mathbb{E}\left(\prod_{i=1}^{k} F_{i}\right) \leq \prod_{i=1}^{k}\left(\mathbb{E}\left(F_{i}\right) \exp \left(C\left|A_{i}\right| e^{-m R}\right)\right)
$$

where $R=\min _{i \neq j} \operatorname{dist}\left(A_{i}, A_{j}\right)$.

Property (a) is familiar for Gibbs states of spin systems with weak interactions. Property (b) is less familiar, but is proven in the Appendix for the class of Gibbs states that correspond to the SRB measures.

Thirdly, $\Gamma$ maps local functions of $\theta$ to local functions of $\omega$ as follows. We say that $w: N^{\mathbb{Z}^{d}} \rightarrow \mathbb{R}$ is local if $w$ is $C^{2}$ with, $\forall z \in \mathbb{Z}^{d}$,

$$
\left\|\frac{\partial w(\theta)}{\partial \theta_{z}}\right\|_{\infty} \leq C(w) \exp (-m|z|)
$$

for some $C(w)<\infty, m>0$. Then one can localize $w \circ \Gamma$ as

$$
(w \circ \Gamma)(\omega)=\sum_{A \subset \mathbb{Z}^{d+1}} w_{A}(\omega)
$$

where $w_{A}$ is supported in $A$ and

$$
\sum_{A \subset \mathbb{Z}^{d+1}}\left|w_{A}\right| e^{\lambda d(A \cup\{0\})}<C(w)
$$

for some $\lambda>0$, and where $d(B)$ is the diameter of the set $B$. This means that $w(\theta)$ becomes under the conjugation a local function of the spins near the space time point 0 . In our problem we will have

$$
\mathbf{J}(x, E, \theta(t))=\mathbf{J}\left(0, \tau_{x} E, \tau_{x} h^{t}(\theta)\right)
$$

where $\tau_{x}$ is the translation by $x \in \mathbb{Z}^{d}$. Since $h^{t} \circ \Gamma=\Gamma \circ \tau^{t}$, we have

$$
\tau_{x} h^{t}(\theta)=\Gamma\left(\tau_{x} \circ \tau^{t}(\omega)\right)
$$

where, on both sides, $\tau_{x}$ denotes the natural action of lattice translations by $x \in \mathbb{Z}^{d}$, and thus $\mathbf{J}\left(0, \tau_{x} E, \tau_{x} h^{t} \circ \Gamma(\omega)\right)$ has an expansion like in (2.6) where in the estimate (2.7) the origin is replaced by the point $(x, t)$ i.e. it is a local function of the spins around $(x, t)$.

2.3. Quenched Diffusion. We may now rephrase the problem of deriving diffusion in deterministic dynamics as that of almost sure, or quenched, diffusion in a random dynamics. Consider the dynamics

$$
E(t+1, x)=E(t, x)+\nabla \cdot \mathbf{J}_{t}(x, E(t), \omega):=f_{t}(x, E(t), \omega)
$$

where $\mathbf{J}_{t}$ is a random field defined on the probability space $(\Omega, \Sigma, \mu)$ described above where $\mu$ satisfies properties (a) and (b). Let us stress that, in our main Theorem 2.1 below, we assume only those properties for $\mu$, plus some symmetries (see (iii) in Section 2.5 below) and nothing else; $\mu$ does not have to correspond to an SRB measure or even to be a Gibbs state. The results for the deterministic dynamical system (1.6, [1.7), stated 
in Corollary 2.3 below, will be consequences of the results on the random dynamics and of (2.6, 2.7).

We want to prove that the function $E(t)$ diffuses almost surely in $\omega$.

Consider first the annealed problem, i.e. the averaged equation (2.9):

$$
E(t+1, x)-E(t, x)=\nabla \cdot \mathbb{E}\left[\mathbf{J}_{t}(x, E(t), \omega)\right]:=\nabla \cdot \mathcal{J}(x, E(t)) .
$$

where, since we will assume below that $\mu$ is invariant under lattice translations (which corresponds for the time direction to the stationarity of $\nu$ ), $\mathcal{J}$ is time independent. Supposing that $h$ and $\mathbf{J}$ have natural symmetries under lattice translations and rotations (see assumption (iii) in section 2.5 below), we infer that $\mathcal{J}$ vanishes at constant $E$ and then, locality assumptions (2.1) that we assumed for $h$ imply

$$
\mathcal{J}(x, E)=\sum_{y} \kappa(x, y, E) \nabla^{\dagger} E(y)
$$

where $\nabla_{\mu}^{\dagger}$ is the adjoint of $\nabla_{\mu}$ in $l^{2}\left(\mathbb{Z}^{d}\right)$, and the matrix $\kappa(x, y, E)$ is a rapidly decaying function of $|x-y|$. Hence, the annealed dynamics is a discrete nonlinear diffusion

$$
E(t+1)-E(t)=\nabla \cdot \kappa(E(t)) \nabla^{\dagger} E(t)
$$

provided the diffusion matrix $\kappa(E(t))$ is positive.

Let now

$$
\beta_{t}(x, E(t))=\mathbf{J}_{t}(x, E(t))-\mathcal{J}(x, E(t))
$$

be the fluctuating part. Then, the $E$-dynamics (2.9) becomes

$$
E(t+1)-E(t)=\nabla \cdot \kappa(E(t)) \nabla^{\dagger} E(t)+\nabla \cdot \beta_{t}(E(t))
$$

with

$$
\mathbb{E} \beta_{t}(E(t))=0 \text {. }
$$

Let us stress that the equations (2.10) and (2.11) are completely general and require only reasonable assumptions of locality and smoothness of the functions $\mathbf{J}$ and $h$ such as the ones we are going to make in section 2.5 below. In particular, the matrix $\kappa$ is close to diagonal (exponentially decaying). In a physical model, one would expect $\kappa(E(t))$ to be positive, although not necessarily uniformly in $E$. If we assume that $\kappa(E(t))$ is uniformly positive in $E$ and that $\beta$ is a small perturbation, then one would expect diffusion to occur almost surely. In what follows, we will make essentially such assumptions on the functions $\mathbf{J}$ and $h$ and prove almost sure diffusion.

2.4. Random Walk in a Random Environment. Before stating the assumptions let us make one more reduction. It is reasonable to assume that $E=0$ is preserved by the dynamics. This then implies $\beta_{t}(0)=0$. Let us study the linearization at $E=0$ :

$$
E(t+1)-E(t)=\nabla \cdot \kappa(0) \nabla^{\dagger} E(t)+\nabla \cdot\left(D \beta_{t}(0) E(t)\right)
$$

where, by symmetry, $\kappa(0)$ is a matrix which is a multiple of the identity (multiple given by $\frac{D_{0}}{2 d}$ in (2.17) below); in other words

$$
E(t+1, x)=\sum_{y} p_{x y}(t) E(t, y)
$$

with

$$
\sum_{x} p_{x y}(t)=1
$$


Since $E \geq 0$ we have $p_{x y} \geq 0$ i.e. $p_{x y}(t)$ are transition probabilities of a random walk. $p_{x y}(t)$ is space and time dependent and random i.e. it defines a random walk in a random environment.

2.5. Assumptions. We prove that under suitable assumptions on $\kappa(0)$ and $D \beta_{t}(0)$ in (2.12) the random dynamical system (2.9) is diffusive $\mu$ a.s. in $\omega$ and $E(0)$ small enough i.e. the deterministic system (1.6), (1.7) is diffusive $\nu$ a.s. in $\theta(0)$.

To state our results, it is convenient to introduce the following notation: we identify $f:\left(L^{-n} \mathbb{Z}\right)^{d} \rightarrow \mathbb{R}$, to a function $f: \mathbb{R}^{d} \rightarrow \mathbb{R}$, which takes the constant value $f(x)$ on $L^{-n}$ cubes in $\mathbb{R}^{d}$ centered at $x \in\left(L^{-n} \mathbb{Z}\right)^{d}$, so that we can write, e.g.:

$$
\int d x f(x):=L^{-n d} \sum_{x \in\left(L^{-n} \mathbb{Z}\right)^{d}} f(x)
$$

Since the $x$ argument of $E(t, x)$ is in $\mathbb{Z}^{d}$, the $x$ argument of $E\left(t, L^{n} x\right)$ is in $\left(L^{-n} \mathbb{Z}\right)^{d}$.

For $f:\left(L^{-n} \mathbb{Z}\right)^{d} \rightarrow \mathbb{R}$, we denote by $\|f\|_{1}$ the $L^{1}\left(\mathbb{R}^{d}\right)$ norm of the associated function $f: \mathbb{R}^{d} \rightarrow \mathbb{R}$. When $n=0$, this coincides with the $\ell^{1}\left(\mathbb{Z}^{d}\right)$ norm.

Let $B_{\delta}^{+}$denote the set of $E(x) \geq 0, x \in \mathbb{Z}^{d}$, with $\|E\|_{1} \leq \delta$. We assume that $f_{t}$ in (2.9) is measurable in $\omega$ and $C^{2}$ in $E$ in $B_{\delta}^{+}$for some $\delta>0$. Moreover, we assume:

(i) Positivity: $f_{t}(x, E, \omega) \geq 0$ for $E \geq 0, \forall x \in \mathbb{Z}^{d}$, and a.s. in $\omega$.

(ii) Conservation law:

$$
\sum_{x \in \mathbb{Z}^{d}} f_{t}(x, E, \omega)=\sum_{x \in \mathbb{Z}^{d}} E(x)
$$

This implies, since $E \geq 0$, that $f(x, 0, \omega)=0$.

It is natural to assume that the randomness is statistically symmetric:

(iii) Symmetry: The law of $f_{t}(x, E, \omega)$ is invariant under the natural action of translations of $\mathbb{Z}^{d+1}$ and rotations fixing $\mathbb{Z}^{d}$.

(iv) Locality: We assume

$$
\begin{aligned}
\left|\partial_{E(y)} f_{t}(x, E, \omega)\right| & \leq C e^{-m|x-y|} \\
\left|\partial_{E(y)} \partial_{E(z)} f_{t}(x, E, \omega)\right| & \leq C e^{-m(|x-y|+|x-z|)}
\end{aligned}
$$

for some $m>0$, uniformly in $\omega$ and $E \in B_{\delta}^{+}$.

Let us write the average of the derivative of the map at $E=0$ as

$$
\mathbb{E}\left(\partial_{E(y)} f_{t}(x, 0, \omega)\right):=T(x-y)
$$

(i.e. $T=1+\nabla \cdot \kappa(0) \nabla^{\dagger}$ in (2.12) $)$. From (i) and (ii), we have $T(x) \geq 0, \sum_{x \in \mathbb{Z}^{d}} T(x)=1$ and from (iv) $T(x) \leq C e^{-m|x|}$. Let $\hat{T}(k)=\sum_{x \in \mathbb{Z}^{d}} T(x) e^{-i k x}$. By assumption (iii), we may write

$$
\hat{T}(k)=1-\frac{D_{0}}{2 d} k^{2}+\mathcal{O}\left(k^{4}\right)
$$

where $D_{0}$ is the diffusion constant

$$
D_{0}=\sum_{u} u^{2} T(u)
$$

We moreover assume: 
(v) $|\hat{T}(k)|<1$ for $k \neq 0$.

This is a convenient assumption: although it is not true for all random walks, in particular for the simple random walk, when all the components of $k$ equal $\pi$, it becomes true when one iterates that walk once.

Write

$$
\partial_{E(y)} f_{t}(x, E, \omega)-T(x-y):=\nabla \cdot b_{t}(x, y, E, \omega)
$$

By (iv) $b_{t}(x, y, 0, \omega)$ decays exponentially in $|x-y|$. We assume that it is local in $\omega$ in the sense of Section 2.2 and small:

(vi) Weak randomness. Assume that

$$
b_{t}(x, y, 0, \omega)=\sum_{A \subset \mathbb{Z}^{d+1}} b_{t, A}(x, y, \omega)
$$

with $b_{t, A}$ supported on $A$ (see Section 2.2) and, for some $\lambda>0$,

$$
\sum_{A \subset \mathbb{Z}^{d+1}, x \in \mathbb{Z}^{d}}\left|b_{t, A}(x, y, \omega)\right| e^{\lambda d(A \cup(x, t) \cup(y, t))}<\epsilon
$$

Below we will usually drop the argument $\omega$.

Then our main result is:

Theorem 2.1. Consider the dynamical system (2.9). Assume that the random field $(\Omega, \Sigma, \mu)$ satisfies (2.3), (2.4) and that $f_{t}$ satisfies the assumptions (i)-(vi) above. Assume moreover that $L$ is an integer, that $d \geq 2$ and that $\epsilon$ and $\delta$ are small enough.

Then there exists, almost surely in $\omega$, and for all $E \in B_{\delta}^{+}, a D>0$ such that, for all $G \in C^{1}\left(\mathbb{R}^{d}\right)$, with $\|G\|_{\infty},\|\nabla G\|_{\infty}$, finite,

$$
\lim _{n \rightarrow \infty} \int d x G(x)\left(L^{n d} E\left(L^{2 n}, L^{n} x\right)-\|E\|_{1} T_{D}^{*}(x)\right)=0
$$

where $T_{D}^{*}(x)=\left(\frac{d}{2 \pi D}\right)^{d / 2} e^{-d x^{2} / 2 D}$.

Remark 2.2. The restriction to $d \geq 2$ is done for convenience. With a more detailed nonlinear analysis (see Section 5) one could extend the result to $d=1$.

Moreover, the result is stated for a subsequence of times of the form $L^{2 n}$, but a general sequence of times and even a result for several times, leading to the convergence of finite dimensional distributions and to an invariance principle, could be obtained along the lines of the arguments in [9].

Finally, the convergence is weak, but is uniform over functions $G$ with fixed norms $\|G\|_{\infty}$ and $\|\nabla G\|_{\infty}$.

Combining this theorem with (2.6), (2.7), we can now formulate a similar result for the deterministic dynamical system (1.6, 1.7). We assume that $F$ in (1.6) satisfies the same assumptions (i), (ii) (iv), (v), stated above for $f_{t}$ in (2.9), and satisfies, instead of (iii),

(iii') Symmetry: $F(x, E(t), \theta(t))$ is invariant under the natural action of translations of $\mathbb{Z}^{d}$ and rotations fixing $\mathbb{Z}^{d}$.

As in (2.18), we define $T(x-y)=\mathbb{E}\left(\partial_{E(y)} F(x, 0, \theta)\right)$ and

$$
\partial_{E(y)} F(x, E, \theta)-T(x-y):=\nabla \cdot b(x, y, E, \theta)
$$


By (iii'), $b(x, y, E, \theta)=b\left(0, y-x, \tau_{x} E, \tau_{x} \theta\right)$. Instead of (vi), we assume for $b$ :

(vi') Weak randomness: We assume that $w(\theta):=b(0, x, 0, \theta)$ is local in the sense (2.5), with $C(w)=\epsilon^{\prime} e^{-m|x|}$.

Then, we have the

Corollary 2.3. Let $g, \psi$ in (1.7) satisfy the assumptions of Section 2.2, with $\kappa$ small enough, and let F in (1.6) satisfy (i), (ii) (iii') (iv), (v), (vi') above. Assume that $\epsilon^{\prime}$ and $\delta$ small enough. Then, the result stated in theorem 2.1 holds also for the dynamical system (1.7) (1.6), almost surely in $\theta$ with respect to the SRB measure $\nu$.

Remark 2.4. Concrete examples of deterministic or random dynamical systems that satisfy the assumptions of Corollary 2.3 or Theorem 2.1 respectively can be obtained by taking in (1.6)

$$
F(x, E, \theta)=E(x)+\sum_{y} d(x-y) E(y)+\tilde{F}(x, E, \theta)
$$

where $d(x) \geq 0$, for $x \neq 0,-\hat{d}(k) / k^{2} \geq d_{0}>0$ and where $\tilde{F}$ is a perturbation as in (vi') with $\epsilon^{\prime}$ small enough. A generic (small, local and $C^{2}$ ) coupling function $\mathbf{J}$ in (1.6) would give rise to $d_{0}$ and $\epsilon^{\prime}$ comparable and hence not satisfying our assumptions. However we expect to be able to handle such cases as well by perturbing around a weak coupling limit i.e. considering first a time scale of order $\epsilon^{-1}$ to produce "effective" $T$ and $b$ that fit into our assumptions [10].

The proofs of Theorem 2.1 and of Corollary 2.3 will be given in section 6 . In the following section, we will explain the Renormalization Group method used in the proofs. In section 4, we discuss the linearized Renormalization Group, and we state and prove the auxiliary Propositions 4.1,4.3,4.5, that are used in Section 6. In section 5, we give the estimates on the nonlinear parts of the Renormalization Group transformation, also used in Section 6 (Proposition 5.1). The Appendix is devoted to properties of Gibbs states and SRB measures that are used in the proof of Corollary 2.3 .

\section{RENORMALIZATION GROUP FOR RANDOM COUPLED MAPS}

The proof of Theorem 2.1 is based on a Renormalization Group (RG) method introduced in [5] and [9]. We will next explain this RG and give an outline of how it yields the Theorem.

3.1. Renormalized Dynamics. Let us introduce the scaling transformation $S_{L}$ :

$$
\left(S_{L} E\right)(x)=L^{d} E(L x) .
$$

where $L>1$. Note that $S_{L} E$ is defined on a finer lattice $\left(L^{-1} \mathbb{Z}\right)^{d}$. Fix $L$ and define, for each $n \in \mathbb{N}$, renormalized energies

$$
E_{n}(t, x)=S_{L^{n}} E\left(L^{2 n} t, x\right),
$$

where $t \in L^{-2 n} \mathbb{N}, x \in\left(L^{-n} \mathbb{Z}\right)^{d}$. We can then rephrase the scaling limit inside the integral in (2.21) as

$$
\lim _{n \rightarrow \infty} L^{n d} E\left(L^{2 n}, L^{n} x\right)=\lim _{n \rightarrow \infty} E_{n}(1, x)
$$


$E_{n}(t)$ inherits its dynamics from the one of $E$. We will call this the renormalized dynamics:

$$
E_{n}(t+1)=f_{n, t}\left(E_{n}(t)\right)
$$

Explicitely we have:

$$
f_{n, t}=S_{L^{n}} \circ f_{L^{2 n} t+L^{2 n}-1} \circ \cdots \circ f_{L^{2 n} t} \circ S_{L^{-n}} .
$$

3.2. Renormalization Group. The dynamics changes with the scale as:

$$
f_{n+1}=\mathcal{R} f_{n},
$$

where

$$
\mathcal{R} f_{n, t}=S_{L} \circ f_{n, t_{L^{2}}} \circ \cdots \circ f_{n, t_{1}} \circ S_{L}^{-1}
$$

with $t_{k}=L^{2} t+k-1, k=1, \ldots L^{2}$. The map $\mathcal{R}$ is the Renormalization Group map acting in a suitable space of random maps $f$. We may rephrase the diffusive scaling limit as a property of the Renormalization Group flow. We will prove that, almost surely, the renormalized maps converge, in a suitable sense,

$$
\mathcal{R}^{n} f \rightarrow f^{*}
$$

where the fixed point is nonrandom and linear:

$$
f^{*}(E)=e^{\frac{D \Delta}{2 d}} E .
$$

$\Delta$ is the Laplacean on $\mathbb{R}^{d}$ (note that $f^{*}$ is defined on $E(x), x \in \mathbb{R}^{d}$ ) and $D>0$. Moreover, we show that the renormalized energies converge almost surely to the fixed point

$$
E_{n}(1, x)-\|E\|_{1}\left(\frac{d}{2 \pi D}\right)^{d / 2} e^{-d x^{2} / 2 D} \rightarrow 0
$$

(in the sense of (2.21) $)$, which is the diffusive scaling limit. These results may be summarized by saying that both the randomness and the nonlinearity are irrelevant in the RG sense. Let us sketch the reasons for this.

3.3. Linearization. Let us adopt the convention that objects on scale $n+1$ are denoted by prime and the unprimed ones are on scale $n$ (and delete the indices $n$ or $n+1$ ). As we will see in Section 6, it will be sufficient to control derivative of $f$ (see (6.2)). For $f^{\prime}=\mathcal{R} f$ the iteration (3.4) implies a recursion for the derivative of $f$ : let $D f$ denote the kernel of the derivative map (using definition (2.14) for the integrals), $D f(x, y)=$ $\partial_{E(y)} f(x, E), x, y \in\left(L^{-n} \mathbb{Z}\right)^{d}$. We get:

$$
D f_{t^{\prime}}^{\prime}\left(E^{\prime}\right)=L^{d} \mathcal{S}_{L}\left(D f_{t_{L^{2}}}\left(E_{L^{2}}\right) D f_{t_{L^{2}-1}}\left(E_{L^{2}-1}\right) \ldots D f_{t_{1}}\left(E_{1}\right)\right)
$$

where

$$
E_{s}=f_{t_{s-1}} \circ \cdots \circ f_{t_{1}}(E), \quad E=L^{-d} E^{\prime}(\cdot / L),
$$

$t_{k}=L^{2} t+k-1, k=1, \ldots L^{2}$. with the convention $E_{1}=E$. $\mathcal{S}_{L}$ is the scaling

$$
\left(\mathcal{S}_{L} M\right)(x, y)=M(L x, L y) .
$$

The factor $L^{d}$ in (3.5) comes from the fact that $D f(x, y)$ is a kernel, the definition (2.14), and the fact that $D f^{\prime}$ on the left hand side of (3.5) is on scale $n+1$ while $D f$ on the right hand side is on scale $n$. 
Let us consider this iteration at $E=0$. Set

$$
p_{t}:=D f_{t}(0) \text {. }
$$

Then

$$
p_{t^{\prime}}^{\prime}\left(x^{\prime}, y^{\prime}\right)=L^{d} p_{t_{L^{2}}} \ldots p_{t_{1}}\left(L x^{\prime}, L y^{\prime}\right) \text {. }
$$

Next, use (2.16), (2.18), to write $p$ as a sum of its average and fluctuation:

$$
p_{t}(x, y)=T(x-y)+\nabla_{n} \cdot b_{t}(x, y)
$$

where $\nabla_{n}$ acts on the $x$ variable and, for a function $f:\left(L^{-n} \mathbb{Z}\right)^{d} \rightarrow \mathbb{R}, \nabla_{n, \mu} f(x)=$ $L^{n}\left(f\left(x+L^{-n} e_{\mu}\right)-f(x)\right)$, and where $b_{t}(x, y)$ is the value of $b_{t}(x, y, E)$ at $E=0$ (we will suppress the index $n$ on $\nabla_{n}$ below, when the scale on which it is defined will be obvious from the context). We have

$$
\mathbb{E} p_{t}(x, y):=T(x-y)
$$

and $\mathbb{E} b_{t}=0$.

Then, for $p^{\prime}=T^{\prime}+\nabla_{n+1} \cdot b^{\prime}$, we get

$$
T^{\prime}\left(x^{\prime}-y^{\prime}\right)=L^{d} T^{L^{2}}\left(L x^{\prime}-L y^{\prime}\right)+P\left(x^{\prime}-y^{\prime}\right)
$$

where $P$ is the expectation of a polynomial in $b$.

For the noise, we get

$$
\begin{aligned}
& \nabla_{n+1} \cdot b_{t^{\prime}}^{\prime}= \\
& L^{d} \sum_{t \in I_{t^{\prime}}} \int d x d y T^{L^{2}-i-1}\left(L x^{\prime}-x\right) \nabla_{n} \cdot b_{t}(x, y) T^{i}\left(y-L y^{\prime}\right)+G_{t^{\prime}},
\end{aligned}
$$

where

$$
I_{t^{\prime}}=\left[L^{2} t^{\prime}, L^{2}\left(t^{\prime}+1\right)-1\right],
$$

$i=t-L^{2} t^{\prime}$ and $G_{t^{\prime}}$ involves quadratic and higher order polynomials in $b$. Note that, since $T$ is time independent, we can write in the sum $T^{L^{2}-i-1}, T^{i}$, where the exponents refer to amounts of time spent in the interval $I_{t^{\prime}}$, but do not depend on $t^{\prime}$, unlike the random variable $b_{t}(x, y)$, where $t \in I_{t^{\prime}}$.

3.4. Linear RG. Treating the noise $b$ as a perturbation, consider first the iterations (3.9) and (3.11) to leading order in $b$. For $b=0$, we get

$$
\mathcal{T}_{n}:=L^{n d} T^{L^{2 n}}\left(L^{n} \cdot\right)
$$

$$
\hat{\mathcal{T}}_{n}(k)=\hat{T}\left(k / L^{n}\right)^{L^{2 n}} .
$$

Then, using (2.17), we get, as $n \rightarrow \infty$ :

$$
\hat{\mathcal{T}}_{n}(k) \rightarrow e^{-\frac{D_{0}}{2 d} k^{2}}:=\hat{T}_{D_{0}}^{*}(k)
$$

which explains the form of the fixed point.

For the noise, the linear RG map is gotten from (3.11) by integrating the $\nabla_{n}$ by parts (actually, Riemann summing by parts) and commuting with $\mathcal{S}_{L}$ (which gives rise to the factor $L^{-1}$, compared to (3.11) ):

$$
(\mathcal{L} b)_{t^{\prime}}=\sum_{t \in I_{t^{\prime}}} \mathcal{L}_{i} b_{t},
$$




$$
\mathcal{L}_{i} b\left(x^{\prime}, y^{\prime}\right):=L^{d-1} \int d x d y T^{L^{2}-i-1}\left(L x^{\prime}-x\right) b(x, y) T^{i}\left(y-L y^{\prime}\right),
$$

with $I_{t^{\prime}}, i$ as in (3.11). In the next Section we will show that a suitable norm of the variance of $b$ contracts under $\mathcal{L}$. Let us explain the intuitive reason for this. Take e.g. $x^{\prime}=y^{\prime}=0$ and $t^{\prime}=0$, the estimate being similar for other $x^{\prime}, y^{\prime}, t^{\prime}$. For $t$ of order $L^{2}$, $T^{t}\left(L x^{\prime}-x\right) \sim L^{-d} e^{-\left|x^{\prime}-x / L\right|}$. Hence the $x$ and the $y$ integrals are localized in an $L$ cube at the origin. As it will turn out, $b_{t}(x, y)$ has exponential decay in $|x-y|, \forall t$, so let us put heuristically $x=y$,

$$
(\mathcal{L} b)(0,0) \sim L^{-d-1} \sum_{t=0}^{L^{2}-1} \int_{|x|<L} d x b_{t}(x, x) .
$$

Since correlations of $b$ decay exponentially in space and time (3.16) is effectively a sum of $L^{d+2}$ independent random variables of variance $L^{-2 d-2}(\mathbb{E} b)^{2}$ and we conclude $\mathbb{E}(\mathcal{L} b)^{2} \sim L^{-d} \mathbb{E} b^{2}$. The actual contraction factor depends on the norm on $b$ and will be slightly different.

Taking into account the corrections $P$ and $G$ in (3.9) and (3.11) that are smaller than the linear term, we conclude that we should expect the variance to contract

$$
\mathbb{E}\left(b_{n, t}\right)^{2} \sim \epsilon_{n}^{2} \rightarrow 0
$$

(for any fixed $t$ ) as $n \rightarrow \infty$ and the iteration of the mean to become

$$
T_{n+1}=L^{d} T_{n}^{L^{2}}(L \cdot)+\mathcal{O}\left(\epsilon_{n}^{2}\right) .
$$

The fixed point is the same but the $\mathcal{O}\left(\epsilon_{n}^{2}\right)$ renormalizes the diffusion constant $D$ at each iteration step (less and less as $n \rightarrow \infty$ ).

3.5. Random Drift. There is a problem however once we try to make this perturbative analysis rigorous. Deterministically the noise is relevant: from (3.16) we see that $\|\mathcal{L} b\|_{\infty}$ can be as big as $\mathcal{O}(L)\|b\|_{\infty}$. This means that there are unlikely events in the environment where the random walk develops a drift. Recall that $b_{n, t}(x, y)$ is the fluctuating part of the transition probability density for random walks in time $L^{2 n}$ from $L^{n} x$ to $L^{n} y$. There will be a (random) region $D_{t} \subset \mathbb{Z}^{d}$ where we write (see (4.22)-(4.29) below for a precise definition), for $u, v \in \mathbb{Z}^{d}$, and any given $t$,

$$
\sup _{x \in \mathbf{u}, y \in \mathbf{v}}\left|b_{n, t}(x, y)\right| \leq \epsilon_{n} e^{N_{n, z}}
$$

with $\mathbf{u}, \mathbf{v}$ the unit cubes in $\mathbb{R}^{d}$ centered on $u, v$ and $z=(t, u)$. Then $N_{n, z}$ can be (very) large, but with (very) small probability:

$$
\operatorname{Prob}\left(N_{n, z}>N\right) \leq e^{-K(n+N)}
$$

with $K$ large (see Proposition 4.3 below for a precise bound).

3.6. Nonlinear corrections. Finally, to control the nonlinear contributions to $f_{n}$ we show that the second derivative $D_{E}^{2} f$ is irrelevant in all dimensions due to the scaling of $E$. Differentiating (3.5) we obtain

$$
D^{2} f_{t^{\prime}}^{\prime}\left(E^{\prime}\right)=L^{d} \sum_{i=1}^{L^{2}} \mathcal{S}_{L}\left(D f^{\left(L^{2}\right)} \ldots D f^{(i+1)} D^{2} f^{(i)}\left(D f^{(i-1)} \ldots D f^{(1)}\right)^{\otimes^{2}}\right)
$$


where we denote $D f^{(i)}=D f_{t_{i}}\left(E_{i}\right)$, and where we write, for matrices $F, G$,

$$
D^{2} f(F \otimes G)(x, y, z)=\int d u d v D^{2} f(x, u, v) F(u, y) G(v, z) .
$$

Evaluating this at $E^{\prime}=0$, and to first order in the noise, we arrive at the linear RG map on $D^{2} f_{t}(0):=\nabla \cdot \rho_{t}$ :

$$
\begin{aligned}
& (\tilde{\mathcal{L}} \rho)_{t^{\prime}}\left(x^{\prime}, y^{\prime}, z^{\prime}\right)= \\
& L^{d-1} \sum_{t \in I_{t^{\prime}}} \int d x d y d z T^{L^{2}-i-1}\left(L x^{\prime}-x\right) \rho_{t}(x, y, z) T^{i}\left(y-L y^{\prime}\right) T^{i}\left(z-L z^{\prime}\right) .
\end{aligned}
$$

with $I_{t^{\prime}}, i$ as in (3.11). We will show in Section 5 that $\tilde{\mathcal{L}}$ is a contraction in a suitable norm. It is not hard to see why this should be so. We will show that $\rho_{t}$ is (exponentially) local, so let us set, heuristically, $x=y=z$. Using the bounds on $T$ (proven in Corollary 4.6 below), $\|T\|_{1}=1$ to control the integral over $x=y=z$, and $\left\|T^{j}\right\|_{\infty}=\mathcal{O}\left(j^{-d / 2}\right)$ for the other powers of $T$, we conclude that the integral yields a smallness factor

$$
\min \left\{i^{-d}, i^{-d / 2}\left(L^{2}-i\right)^{-d / 2}\right\} \leq 2^{d / 2} L^{-d} i^{-d / 2},
$$

whose sum over $i \in\left[1, L^{2}\right]$, multiplied by $L^{d-1}$, is small for $d>1$, i.e. we expect $\tilde{\mathcal{L}}$ to be contractive for $d>1$.

\section{RANDOM WALK in a RANDOM EnVIRONMENT}

We saw in Section 3.3 that the linearization of our random dynamical system, $p_{t}=$ $D f_{t}(0)$, has a closed evolution (3.6) under the RG. Thus we solve this first and use the result as a driving term for the nonlinear part of $f_{t}$. $p_{t}$ defines a random walk in a random environment that has exponentially decaying correlations in both space and time. An almost sure (or quenched) central limit theorem has been established for this problem [16, 15, 9], but we need a very detailed knowledge of it in order to control the nonlinear flow. The proof given in this section is a (much simplified) version of the (much more difficult) proof [5] for environments that are time independent.

4.1. Localization. Recall from Section 2.5 that the fluctuating part of the map $f_{t}$ has the expansion (2.19) in terms of localized terms that are (almost) independent for disjoint $A$ 's. As explained in Section 3.5. there will be a random region $D_{t} \subset \mathbb{Z}^{d}$ of possibly large drift. It turns out to be useful to localize the $b$ on the set $D_{t}$. We will iterate a representation

$$
b_{t}=\sum_{\mathcal{D} \subset D_{t}} \sum_{A \subset \mathbb{Z}^{d+1}} b_{t, \mathcal{D}, A}
$$

Note also that $\mathcal{D}=\emptyset$ is included (and is actually the more probable contribution). The representation (2.19) is of this form, with $b_{t, \mathcal{D}, A}=0$, if $\mathcal{D} \neq \emptyset$. We will denote

$$
s_{t, A}:=b_{t, \emptyset, A}
$$

and write

$$
b_{t}=s_{t}+\ell_{t}
$$

("small" and "large" contributions, the $s$ term being more probable and the $\ell$ one including the large, but rare, contributions). Given $b_{t}, D_{t}$, we need to explain how 
$b_{t^{\prime}}^{\prime}, D_{t^{\prime}}^{\prime}$ are defined in terms of them. This will need several steps and definitions, to which we turn now.

4.2. Relevant and irrelevant variables. Let us first discuss the linear RG (3.15). Thus let $\sigma=\mathcal{L} s$ i.e.

$$
\sigma_{t^{\prime}}=\sum_{t \in I_{t^{\prime}}} \sum_{A \subset \mathbb{Z}^{d+1}} \mathcal{L}_{i} s_{t, A}
$$

where

$$
I_{t^{\prime}}=\left[L^{2} t^{\prime}, L^{2}\left(t^{\prime}+1\right)-1\right]
$$

and $i=t-L^{2} t^{\prime}$ as in (3.11). Next, we localize $\sigma$. We introduce some notation. Given $z^{\prime} \in \mathbb{Z}^{d+1}$ let $\mathbf{z}^{\prime}$ denote the unit cube in $\mathbb{R}^{d+1}$ with center $z^{\prime}$. Define also "blocking": given $A \subset \mathbb{Z}^{d+1}$ set

$$
[A]:=\operatorname{int}\left\{\left(L^{-2} t, L^{-1} u\right) \mid(t, u) \in A\right\}
$$

where int denotes the integer part. Given a subset $A^{\prime}$ of $\mathbb{Z}^{d+1}$ we define a family $\mathcal{A}\left(A^{\prime}\right)$ of subsets $A$ of $\mathbb{Z}^{d+1}$ as follows. Let first $\left|A^{\prime}\right|=1$ i.e. $A^{\prime}=z^{\prime} \in \mathbb{Z}^{d+1}$ (by a slight abuse and in order to simplify notations, we write $z^{\prime}$ instead of $\left.\left\{z^{\prime}\right\}\right)$. Then $A \in \mathcal{A}\left(z^{\prime}\right)$ if either $[A] \subset \mathbf{z}^{\prime}$, or $[A] \cap z^{\prime} \neq \emptyset$, $\operatorname{diam}(A) \leq \frac{L}{4}$ and the first point $z$ (in lexicographical order) in $[A]$ belongs to $\mathbf{z}^{\prime}$. For $\left|A^{\prime}\right|>1$ we let $A \in \mathcal{A}\left(A^{\prime}\right)$ if $[A]=A^{\prime}$ and $\operatorname{diam}(A)>\frac{L}{4}$. Then, defining

$$
\sigma_{t^{\prime}, A^{\prime}}=\sum_{t \in I_{t^{\prime}}} \sum_{A \in \mathcal{A}\left(A^{\prime}\right)} \mathcal{L}_{i} s_{t, A}
$$

we have

$$
\sigma_{t^{\prime}}=\sum_{A^{\prime}} \sigma_{t^{\prime}, A^{\prime}}
$$

The virtue of this decomposition is that, as we will see in (4.88) below, the nonlocal terms $\sigma_{A}$ with $|A|>1$ will strongly contract.

The term will $|A|=1$ does not contract deterministically, but it will contract with high probability, basically because its variance contracts, as explained in (3.16). We will come back to this lack of deterministic contraction in Section 4.4.

4.3. Composite operators. Let us next turn to the remainder term $G_{t^{\prime}}$ in (3.11) consisting of a polynomial in $b$. We introduce some notation. For matrices $F_{t}(x, y)$, indexed by time, we denote

$$
F_{I}=F_{t} F_{t-1} \ldots F_{s}
$$

for an interval of times $I=[s, t]$. Let $\mathcal{P}$ be the set of nontrivial partitions $\pi$ of the time interval $I_{t^{\prime}}$ into intervals $\pi_{1}, \ldots, \pi_{k}$ arranged in increasing time order and $\pi_{1}, \pi_{k}$ possibly empty, with $T_{\pi_{k}}, T_{\pi_{1}}$ being then the identity (thus $k \leq L^{2}+2$ ). Then,

$$
p^{\prime}=L^{d} \mathcal{S}_{L} T^{L^{2}}+R^{\prime}
$$

with

$$
R^{\prime}=L^{d} \sum_{\pi \in \mathcal{P}} \mathcal{S}_{L} T_{\pi_{k}}\left(\nabla_{n} \cdot b\right)_{\pi_{k-1}} T_{\pi_{k-2}} \ldots\left(\nabla_{n} \cdot b\right)_{\pi_{2}} T_{\pi_{1}}
$$


(remembering that $T_{\pi_{k}}$ or $T_{\pi_{1}}$ could be the identity). Thus, writing $\pi_{i}=\left\{\tau_{i}\right\} \cup \pi_{i}^{\prime}$ with $\tau_{i}$ the largest time, we get, by integration by parts,

$$
R^{\prime}=\nabla_{n+1} \cdot Q^{\prime}
$$

with

$$
Q^{\prime}=L^{d-1} \sum_{\pi \in \mathcal{P}} \mathcal{S}_{L} T_{\pi_{k}} b_{\tau_{k-1}}\left(\nabla_{n} \cdot b\right)_{\pi_{k-1}^{\prime}} \nabla_{n} \cdot T_{\pi_{k-2}} \ldots b_{\tau_{2}}\left(\nabla_{n} \cdot b\right)_{\pi_{2}^{\prime}} T_{\pi_{1}}
$$

where the $L^{-1}$ comes from the commutation of $\nabla$ and $\mathcal{S}_{L}$, as in (3.15). Note that, by integration by parts, we have transferred the action of one $\nabla_{n}$ from a $b$ to a $T$. Thus, we never encounter factors like $T \nabla_{n} \cdot b \nabla_{n} \cdot b$ or $\nabla_{n} \cdot T \nabla_{n} \cdot b$. It turns out that there is nevertheless an obstruction to bounding these nonlinear terms due to the gradients in (4.11). Indeed, we will show (see Proposition 4.1) that, in a suitable norm,

$$
\|s\| \leq\left(L^{-n} \epsilon\right)^{\zeta}
$$

with $0<\zeta<1$. This bound is not sufficient to show that the nonlinear terms in eq. (4.11) (with $b$ replaced by $s$ ) are smaller than the linear one. Indeed, on the lattice $\left(L^{-n} \mathbb{Z}\right)^{d}$ the operator $\nabla_{n}$ is bounded in sup norm by $C L^{n}$ and hence $\left\|\nabla_{n} \cdot s\right\| \leq$ $C L^{(1-\zeta) n} \epsilon^{\zeta}$, which is large. To show that the nonlinear terms are subleading, we need to show that $\left\|s \nabla_{n} \cdot s\right\|$ has a better bound than $\|s\|$ and that $\left\|\nabla_{n} \cdot s \nabla_{n} \cdot s\right\|$ is also small (in Proposition 4.1 below we will state bounds on these variables and, in subsection 4.10, we will show that this is enough to control all the nonlinear terms). In Renormalization Group language, we need to show that these "composite operators" are irrelevant. Hence, let us look at the linear RG for them.

Let

$$
B_{t}:=b_{t+1} \nabla_{n} \cdot b_{t}
$$

Inserting the expansions (4.11) and using

$$
\left(\mathcal{S}_{L} c\right)\left(\mathcal{S}_{L} d\right)=L^{-d} \mathcal{S}_{L}(c d)
$$

which follows by a change of variables, we observe that $B_{t^{\prime}}^{\prime}$ is given by the expansion (4.11) on the time interval $I_{t^{\prime}} \cup I_{t^{\prime}+1}$ with the additional constraint that there is at least one factor of $b$ on both intervals $I_{t^{\prime}}$ and $I_{t^{\prime}+1}$. In the terms in (4.11) involving only two consecutive $b$ 's, the only one involving $\nabla_{n} \cdot b$ is the linear RG one (because the last $b$ factor in each term in (4.11) is $b_{\tau_{k-1}}$ and not $\nabla_{n} \cdot b_{\tau_{k-1}}$ ):

$$
(\mathcal{L} B)_{t^{\prime}}:=L^{d-1} \mathcal{S}_{L} T^{L^{2}-1} B_{L^{2}\left(t^{\prime}+1\right)-1} T^{L^{2}-1},
$$

and it turns out that this linear RG will be contractive in our norm.

The localization of $B$ is inherited from the one of $b$ in (4.1). We get

$$
B_{t}=\sum_{\mathcal{D}} \sum_{A \subset \mathbb{Z}^{d+1}} B_{t, \mathcal{D}, A}
$$

where now $\mathcal{D}:=\left(\mathcal{D}_{t}, \mathcal{D}_{t+1}\right)$ with $\mathcal{D}_{t} \subset D_{t}, \mathcal{D}_{t+1} \subset D_{t+1}$ and

$$
B_{t, \mathcal{D}, A}=\sum_{A_{t+1}, A_{t}} b_{t+1, \mathcal{D}_{t+1}, A_{t+1}} \nabla_{n} \cdot b_{t, \mathcal{D}_{t}, A_{t}}
$$

with $A_{t} \cup A_{t+1}=A$. 
4.4. Drift region. The drift region is inductively built out of the regions where $\sigma=\mathcal{L} s$ fails to contract. Let us denote the local part of $s$ by $s^{l o c}$, i.e.

$$
s_{t}^{l o c}:=\sum_{u \in \mathbb{Z}^{d}} s_{t, u} .
$$

and

$$
\sigma_{t^{\prime}}^{1}:=\sum_{u^{\prime} \in \mathbb{Z}^{d}}\left(\mathcal{L} s^{l o c}\right)_{t^{\prime}, u^{\prime}}
$$

Here and below, when we use the indices $t, u$, we mean them as in (4.2), i.e. with $\mathcal{D}=\emptyset$, and with $|A|=1, A=\{u\}$.

The size of $b_{n}$ will be measured in terms of a running parameter

$$
\epsilon_{n}=L^{-n} \epsilon
$$

where $\epsilon$ is a bound on the size of the initial $b$, see (2.20). We also need a parameter $\gamma$ satisfying

$$
0<\gamma<1 / 12
$$

Given a space-time point $z^{\prime}=\left(t^{\prime}, u^{\prime}\right)$ define random variables

$$
r_{z^{\prime}}:=\left(n_{0}+n\right) \log L \text { if }\left\|\sigma_{t^{\prime}, u^{\prime}}^{1}\right\|_{\lambda} \geq \frac{1}{8} \epsilon_{n+1}^{1-3 \gamma}
$$

and zero otherwise, where the norm $\|\cdot\|_{\lambda}$ is defined in (4.29) below. The number $n_{0}$ is chosen so that:

$$
L^{-n_{0}}=\epsilon^{3 \gamma}
$$

$D_{t}$ is now defined in terms of random integers $N_{n, z}, z=(t, u) \in \mathbb{Z}^{d+1}$ and where we usually suppress the index $n$ (and, as before, primes will refer to the scale $n+1$ )

$$
D_{t}=\left\{u \in \mathbb{Z}^{d} \mid N_{(t, u)} \neq 0\right\}
$$

which, in turn, are given recursively in terms of the random variables $r$ of previous scales as follows. Let

$$
\tilde{N}_{z^{\prime}}=\sum_{z:[z]=z^{\prime}} N_{z}
$$

where we write $z$ for $\{z\}$ and the blocking operation $[z]$ is defined in (4.5). Then we set

$$
\begin{gathered}
N_{z^{\prime}}^{\prime}:=\tilde{N}_{z^{\prime}}-1+r_{z^{\prime}} \text {, if } \tilde{N}_{z^{\prime}} \geq \lambda / 3 \text { or } r_{z^{\prime}} \neq 0 \\
N_{z^{\prime}}^{\prime}=0, \text { if } \tilde{N}_{z^{\prime}}<\lambda / 3 \text { and } r_{z^{\prime}}=0 .
\end{gathered}
$$

The subtraction by one means that, if no large fluctuations $r_{z^{\prime}}$ occur during a sufficient number of scales, then $N$ will eventually vanish. 
4.5. Norms. We will iterate bounds for the $b_{t}$ and $B_{t}$. For this, we need to introduce suitable norms. For a kernel $b(x, y)$ with $x, y \in L^{-n} \mathbb{Z}^{d}$ let, for $u, v \in \mathbb{Z}^{d}$

$$
\|b\|_{u, v}=\sup _{x \in \mathbf{u}, y \in \mathbf{v}}|b(x, y)| .
$$

Strictly speaking, the norm depends on $n$, but we will suppress that in the notation (we always use unprimed variables for objects on scale $n$ and primed ones for objects on scale $n+1)$. Define, for $C \subset \mathbb{Z}^{d}, \tau(C)$ to be the minimal number of edges of a connected graph whose vertex set is $C$ and whose edges are nearest neighbor bonds in $\mathbb{Z}^{d}$, and denote by $d(A)$ the diameter of a set $A \subset \mathbb{Z}^{d+1}$. Set

$$
l(\mathcal{D}, A, t, u, v):=\tau(\mathcal{D} \cup u \cup v)+d(A \cup(t, u) \cup(t, v)) .
$$

Let us also define, for $\mathcal{D} \subset \mathbb{Z}^{d}$,

$$
N_{t}(\mathcal{D}):=\sum_{u \in \mathcal{D}} N_{(t, u)}
$$

and, for $\mathcal{D}=\left(\mathcal{D}_{1}, \mathcal{D}_{2}\right)$, let analogously $N_{t}(\mathcal{D}):=N_{t}\left(\mathcal{D}_{1}\right)+N_{t+1}\left(\mathcal{D}_{2}\right)$. Now, define the norm

$$
\left\|b_{t}\right\|_{\lambda}=\sup _{v \in \mathbb{Z}^{d}} \sum_{\mathcal{D}, A, u}\left\|b_{t, \mathcal{D}, A}\right\|_{u, v} e^{\lambda l(\mathcal{D}, A, t, u, v)} e^{-N_{t}(\mathcal{D})} .
$$

For $B$ we use the same formula with the difference that the argument of $\tau$ in (4.27) is replaced by $\mathcal{D}_{t} \cup \mathcal{D}_{t+1} \cup u \cup v$.

4.6. Definition of $b^{\prime}$ and $B^{\prime}$. We are now ready to give the inductive construction of $b_{t^{\prime}, D^{\prime}, A^{\prime}}^{\prime}$ and $B_{t^{\prime}, D^{\prime}, A^{\prime}}^{\prime}$. To have a unified notation, set $B^{1}:=b, B^{2}:=B, B^{3}:=\nabla \cdot B$, $B^{4}:=T$ and $B^{5}:=\nabla T$, with the convention that $B^{4}, B^{5}$ are zero if either $\mathcal{D}$ or $A$ is not empty. Then (3.7), (4.8), (4.10) and (4.11) give, using the shorthand $\mathbb{E}^{\perp}:=1-\mathbb{E}$,

$$
b^{\prime}=\mathcal{L} b+\mathbb{E}^{\perp} \mathcal{N}^{1},
$$

where $\mathcal{L}$ is defined in (3.15), and $\mathcal{N}^{1}$ is a sum of products

$$
\Pi=L^{d-1} \mathcal{S}_{L} B_{t_{1}}^{\alpha_{1}} \ldots B_{t_{N}}^{\alpha_{N}}
$$

with $N \leq L^{2}$, that are at least quadratic in $b$ (where we count $B$ as quadratic). As a consequence of (4.30), (3.7), (3.8), we have

$$
T^{\prime}=L^{d} \mathcal{S}_{L} T^{L^{2}}+\mathbb{E} \nabla \cdot \mathcal{N}^{1}
$$

In the same way, we get

$$
B^{\prime}=\mathcal{L} B+\mathcal{N}^{2}
$$

with $\mathcal{L}$ given by (4.14), and $\mathcal{N}^{2}$ is a sum of products $\Pi$, as in (4.31), with $N \leq 2 L^{2}$ and at least one $b$ on each $L^{2}$ interval. In both $\mathcal{N}^{1}, \mathcal{N}^{2}, \alpha_{1} \neq 3,5$ (because the products $\Pi$ do not start with a $\nabla$, see (4.11) ) .

Also, it suffices to give the definitions for $t^{\prime}=0$, so that the times $t_{i}$ lie on $\left[0, L^{2}-1\right]$ for $b^{\prime}$ and on $\left[0,2 L^{2}-1\right]$ for $B^{\prime}$. For simplicity we suppress the index $t^{\prime}$ in the notation.

We need to localize these expansions to get the primed versions of (4.1) and (4.15). For this, insert the decompositions (4.1) and (4.15) to (4.31). The result is localized basically by taking unions of the $\mathcal{D}_{t}$ and $A_{t}$ and blocking the result, except that we need to take care of the constraints, in (4.1), (4.15), $\mathcal{D}^{\prime} \subset D^{\prime}$. Remember that, because 
of (4.25), $D^{\prime}$ can be a smaller than the blocking of $D$. This means that, at each step, parts of the large fields may become small, due to the fact that $N_{z^{\prime}}^{\prime}$ may become zero, see (4.25), and have therefore to be reabsorded into the small fields (see (4.42) below).

Write in (4.31)

$$
b_{t_{i}}=\sum_{\mathcal{D}_{i} \subset \mathcal{D}_{t_{i}}} \sum_{A_{i}} b_{t_{i}, \mathcal{D}_{i}, A_{i}}=\sum_{\tilde{\mathcal{D}}_{i} \subset D_{t_{i}} \cap L D^{\prime}} \sum_{\tilde{A}_{i}} \tilde{b}_{i \tilde{\mathcal{D}}_{i} \tilde{A}_{i}}
$$

with

$$
\tilde{b}_{i \tilde{\mathcal{D}}_{i} \tilde{A}_{i}}:=\sum_{\mathcal{D}_{i} \subset D_{t_{i}}}^{\prime} \sum_{A_{i}}^{\prime} b_{t, \mathcal{D}_{i}, A_{i}}
$$

where the sums are constrained by

$$
\mathcal{D}_{i} \cap L D^{\prime}=\tilde{\mathcal{D}}_{i} \text { and } A_{i} \cup\left(\mathcal{D}_{i} \backslash \tilde{\mathcal{D}}_{i}\right)=\tilde{A}_{i} .
$$

where $L D^{\prime}=\left\{x \in \mathbb{Z}^{d} \mid[x] \in D^{\prime}\right\}$. For $B$ and $\nabla \cdot B$ we proceed the same way except that, this time, $\mathcal{D}_{i}$ is a pair $\left(\mathcal{D}_{t_{i}}, \mathcal{D}_{t_{i}+1}\right)$. This way we end up with

$$
\Pi=L^{d-1} \sum \mathcal{S}_{L} \tilde{B}_{1 \tilde{\mathcal{D}}_{1} \tilde{A}_{1}}^{\alpha_{1}} \ldots \tilde{B}_{N \tilde{\mathcal{D}}_{N} \tilde{A}_{N}}^{\alpha_{N}}
$$

where the sum runs over $\tilde{\mathcal{D}}_{i}, \tilde{A}_{i}$ and, by convention, $\tilde{B}^{\alpha_{i}}=B^{\alpha_{i}}$ for $\alpha_{i}=4,5$. Now, we may localize

$$
\Pi=\sum_{\mathcal{D}^{\prime} \subset D^{\prime}, A^{\prime}} \Pi_{\mathcal{D}^{\prime} A^{\prime}}
$$

with $\Pi_{\mathcal{D}^{\prime} A^{\prime}}$ being a sum of terms in (4.37) with

$$
\mathcal{D}^{\prime}=\left[\cup_{i} \tilde{\mathcal{D}}_{i}\right], \quad A^{\prime}=\left[\cup_{i} \tilde{A}_{i}\right]
$$

Our notation is a bit abusive: if $\alpha_{i}$ equals 2 or 3 , both sets in the pair $\tilde{\mathcal{D}}_{i}$ occur in the union. Since $\mathcal{N}^{1}$ is a sum of $\Pi^{\prime} s$, (4.38) yields a similar expansion for $\mathcal{N}^{1}$.

We still need to localize $\mathcal{L} b$. For $\mathcal{L} s=\sigma$ this was done in Section 4.2. For $\mathcal{L} \ell$ we proceed as above with $\mathcal{N}^{1}$; now all $\alpha_{i}=4$ except for one which equals 1 . We get

$$
\mathcal{L} \ell=\sum_{\mathcal{D}^{\prime} \subset D^{\prime}, A^{\prime}}(\mathcal{L} \ell)_{\mathcal{D}^{\prime} A^{\prime}}
$$

with

$$
(\mathcal{L} \ell)_{\mathcal{D}^{\prime} A^{\prime}}=\sum_{t=0}^{L^{2}-1} \sum_{\tilde{\mathcal{D}}_{t} \neq \emptyset} \sum_{\tilde{A}_{t}} \mathcal{L}_{t} \tilde{\ell}_{t, \tilde{\mathcal{D}}_{t}, \tilde{A}_{t}}
$$

where $\tilde{\ell}$ is defined in terms of $\ell$ as in (4.35), $\tilde{\mathcal{D}}_{t} \subset D_{t} \cap L D_{t^{\prime}}^{\prime}, \mathcal{D}^{\prime}=\left[\tilde{\mathcal{D}}_{t}\right], A^{\prime}=\left[\tilde{A}_{t}\right]$ and $\mathcal{L}_{t}$ is given by (3.15).

Let $\chi_{A^{\prime}}=1$ if $A^{\prime}=z^{\prime}$ and $r_{z^{\prime}}=0$, and 0 otherwise. We define:

$$
s_{A^{\prime}}^{\prime}=\mathbb{E}^{\perp}\left(\sigma_{A^{\prime}}^{1} \chi_{A^{\prime}}+\sigma_{A^{\prime}}^{2}+(\mathcal{L} \ell)_{\emptyset A^{\prime}}+\mathcal{N}_{\emptyset A^{\prime}}^{1}\right),
$$

where $\sigma^{2}$ is defined by

$$
\mathcal{L} s=\sigma^{1}+\sigma^{2}
$$


and $\sigma^{1}$ is the local part given in (4.17); we define also:

$$
\ell_{\mathcal{D}^{\prime} A^{\prime}}^{\prime}=\mathbb{E}^{\perp}\left((\mathcal{L} \ell)_{\mathcal{D}^{\prime} A^{\prime}}+\sigma_{\mathcal{D}^{\prime}}^{1}\left(1-\chi_{\mathcal{D}^{\prime}}\right) \delta_{A^{\prime}, \emptyset}+\mathcal{N}_{\mathcal{D}^{\prime} A^{\prime}}^{1}\right) .
$$

It is easy to verify that, with these definitions,

$$
b^{\prime}=s^{\prime}+\ell^{\prime}
$$

with $b^{\prime}$ given by (4.30).

Both $s^{\prime}$ and $\ell^{\prime}$ have a linear and a nonlinear part. Besides, when one goes from one scale to the next, there is a new large field arising from the previous scale small field and a part of the previous scale large field that becomes small. The factor $\chi_{A^{\prime}}$ in (4.42) ensures that the contribution coming from the local part of $s$ will be small, and the remaining part $\sigma_{\mathcal{D}^{\prime}}^{1}\left(1-\chi_{\mathcal{D}^{\prime}}\right) \delta_{A^{\prime}, \emptyset}$ contributes to the new large field and is thus put into $\ell^{\prime}$. The term $(\mathcal{L} \ell)_{\emptyset A^{\prime}}$ corresponds to the part of previous scale large fields that have become small (due to the decrease of $N$ in (4.24-4.25) ), and which thus contribute to $s^{\prime}$.

For $B^{\prime}$ we need no special treatment of the linear term as we did for $\mathcal{L} b$ and both terms in (4.33) are localized in the same way by expressing $B$ in terms of $\tilde{B}$ as in (4.35) and collecting terms with (4.39). We get

$$
B_{\mathcal{D}^{\prime} A^{\prime}}^{\prime}=(\mathcal{L} B)_{\mathcal{D}^{\prime} A^{\prime}}+\mathcal{N}_{\mathcal{D}^{\prime} A^{\prime}}^{2}
$$

Next, we will state the estimates on the linearized RG, that will be used in Section 6, when we prove Theorem 2.1. Proposition 4.1, which gives deterministic estimates on the random part $b$ of transition probabilities of our random walk will be proven in subsection 4.10; because of the $e^{-N_{t}(\mathcal{D})}$ factor in (4.29), these bounds are useful only if non-zero values of $N$ are improbable, which is the content of Proposition 4.3. This Proposition will be proven in subsection 4.12; this proof uses Proposition 4.4, which itself is proven in subsection 4.11. Proposition 4.5 deals with the nonrandom part of the transition probabilities, given by (3.8), and is proven in subsection 4.13.

4.7. Deterministic bounds. Let us now state the bounds that will be proven inductively in the scale $n$ for $b$ and $B$. Recalling the definition (4.16), decompose $s=s^{\text {loc }}+s^{\text {nloc }}$. We suppress both the index $n$ and the index $t$ in our notation. The number $\gamma$ has been introduced in (4.19) and $\lambda$ is chosen small enough so that (2.20) holds for $n=0$, and that the bound (4.57) below also holds.

Proposition 4.1. For $\epsilon$ small enough we have, $\forall n \in \mathbb{N}, \forall t \in \mathbb{N}$,

$$
\begin{aligned}
\left\|s^{\text {loc }}\right\|_{\lambda} & \leq \epsilon_{n}^{(1-3 \gamma)} \\
\left\|s^{\text {loc }}\right\|_{\lambda} & \leq \epsilon_{n}^{(1-2 \gamma)} \\
\|\ell\|_{\lambda} & \leq \epsilon_{n}^{(1-\gamma)}
\end{aligned}
$$

Moreover

$$
\begin{aligned}
\|B\|_{\lambda} & \leq \epsilon_{n}^{(1-\gamma / 2)} \\
\|\nabla \cdot B\|_{\lambda} & \leq \epsilon_{n}^{1 / 2} .
\end{aligned}
$$

We note also a simple consequence of (4.46)-(4.48) and the fact that, since the lattice spacing is $L^{-n}$, the norm of $\nabla_{n}$ is bounded by $C L^{n}$ :

$$
\|\nabla \cdot b\|_{\lambda} \leq C \epsilon \epsilon_{n}^{-3 \gamma} \text {. }
$$


Remark 4.2. The different powers introduced in the Proposition are chosen for convenience and are not optimal.

Proposition 4.1 is a deterministic statement holding for any realization of the noise. For this statement to be useful, we need to show (see the definition (4.29) of our norm) that the random variables $N_{z}$ are zero with high probability.

4.8. Probabilistic bounds. Our main estimate is:

Proposition 4.3. The random integers $N_{n, z}$, defined in (4.20]4.25), satisfy for any $A \subset \mathbb{Z}^{d+1}$, any $\left\{N_{z}\right\}_{z \in A}$, with $N_{z} \neq 0$,

$$
\mathbb{P}\left(\left\{N_{n, z}=N_{z}\right\}_{z \in A}\right) \leq \exp \left(-K_{n} n|A|-2 K_{n} \sum_{z \in A} N_{z}\right)
$$

with $K_{n}=K \prod_{\ell=2}^{n-1}\left(1-\ell^{-2}\right), n \geq 3, K_{n}=K, n<3$, and where $K \rightarrow \infty$ as $\epsilon \rightarrow 0$.

Since $N$ is determined in terms of the random variables $r$ in previous scales this Proposition follows ultimately from an estimate on the probability that $r_{z} \neq 0$. This in turn follows from the control of the variance of deterministically relevant part of $s$, i.e. $s^{\text {loc }}$. Given $u \in \mathbb{Z}^{d}$ consider the random variables

$$
\left(s_{t, u}, f\right):=\int d x d y s_{t, u}(x, y) f(x, y)
$$

with $f: \mathbb{R}^{d} \times \mathbb{R}^{d} \rightarrow \mathbb{R}$ a measurable function such that

$$
\|f\|_{u}:=\int d x d y|f(x, y)| \exp (-2 \lambda \tau(u, x, y))<\infty .
$$

Here, again by an abuse of notation, we write $\tau(u, x, y)$ instead of $\tau(\{u, v, w\})$, with $x \in \mathbf{v} y \in \mathbf{w}$ (since $\tau$ is defined on sets). Then we prove the following exponential moment estimate:

Proposition 4.4. For all $t \in \mathbb{N}, u \in \mathbb{Z}^{d}$

$$
\mathbb{E} e^{\left(s_{t, u}, f\right)} \leq e^{\delta_{n}\|f\|_{u}^{2}},
$$

with $\delta_{n}=L^{-n \gamma} \epsilon_{n}^{2-6 \gamma}$.

Note that (4.55) implies, by replacing $f$ by af, subtracting 1 on both sides, dividing by $a^{2}$, letting $a \rightarrow 0$ and using $\mathbb{E} s_{t, u}(x, y)=0$ :

$$
\mathbb{E}\left(s_{t, u}, f\right)^{2} \leq \delta_{n}\|f\|_{u}^{2} .
$$

4.9. Inductive bounds for $T$. The following result describes the inductive bounds for the average (3.8) of $p$. We can view $T$ as being of the form (4.1), with the only nonzero term corresponding to $A=\emptyset, \mathcal{D}=\emptyset$, so that $\|T\|_{\lambda}$ is bounded from above and below by $C \int T(x) e^{\lambda|x|} d x$.

Proposition 4.5. $T=T_{n}$ satisfies uniformly in $n$

$$
\begin{aligned}
& \|T\|_{\lambda}+\|\nabla T\|_{\lambda} \leq C \\
& 0 \leq T^{t}(x) \leq C(k)(t+1)^{-d / 2} e^{-c(t)|x|},
\end{aligned}
$$


for $\frac{k}{L} \leq \frac{\lambda}{2}$, and $t \in\left[0,2 L^{2}\right]$, with $c(t)=\min \left(\lambda, \frac{4 k}{\sqrt{t+1}}\right)$. Moreover, there exists $D>0$ such that

$$
\lim _{n \rightarrow \infty}\left\|T_{n}-T_{D}^{*}\right\|_{1}=0
$$

A consequence of (4.58) is:

Corollary 4.6. Let $\tilde{T}^{t}(x):=e^{\frac{k}{L}|x|} T^{t}(x)$. Then, for $\frac{k}{L} \leq \frac{\lambda}{2}$ and $t \in\left[0,2 L^{2}\right]$ we have $\frac{k}{L} \leq \frac{1}{2} c(t)$, and so,

$$
\left\|\tilde{T}^{t}\right\|_{\infty} \leq C(k)(t+1)^{-\frac{d}{2}}, \quad\left\|\tilde{T}^{t}\right\|_{1} \leq C(k) .
$$

Remark 4.7. In the proofs below we shall use the following conventions. $C$ or $c$ denote constants that may vary from place to place, even in the same equation, but that do not depend on $L$ or on the scale $n$. $C(L)$ is similar, but depends on $L$ but not on $n$. We assume throughout that $L$ has been chosen large enough so that inequalities of the form $C L^{-\gamma} \leq 1$, for $\gamma>0$, can be assumed. We then choose $\epsilon$ small enough so that we can assume inequalities of the form $C(L) \epsilon^{\gamma} \leq 1$.

Remark 4.8. In the proofs below, whenever the index $t^{\prime}$ does not appear, it means that it is set equal to 0 . Then, in (3.11), the interval $I_{t^{\prime}}=\left[0, L^{2}-1\right]$ and $i=t$. When considering composite operators we have $t \in\left[0,2 L^{2}-1\right]$.

4.10. Proof of Proposition 4.1. For $n=0$, we have, by assumption (2.20),

$$
\|b\|_{\lambda} \leq \epsilon, \quad\|B\|_{\lambda} \leq C \epsilon^{2} .
$$

Thus, since for $n=0$ the norm of $\nabla_{0}$ is of order one, the assumptions hold for small $\epsilon$ and we have $\mathcal{D}=\emptyset, N=0, \ell=0$.

Before proceeding to the induction, let us sketch the main steps of the proof. For the nonlinear terms in (4.42, 4.44), we need only to show that the norm of a product of $b$ 's, $b \nabla \cdot b$ 's and $\nabla \cdot b \nabla \cdot b$ 's is suitably bounded in terms of the product of their norms (all terms of the form $\Pi$ in (4.31) can be written as a product of those three factors times $T$ 's or $\nabla T$ 's whose norms are bounded by (4.57)), that the resummation (4.35) does not increase the norm too much, and then use inductively Proposition 4.1. This is done in Lemma 4.9 below and involves only simple estimates on the exponential factors in (4.29).

For the linear terms in (4.42),$\sigma_{A^{\prime}}^{1} \chi_{A^{\prime}}$ is controlled trivially because of the characteristic function, the norm of $\sigma_{A^{\prime}}^{2}$, being nonlocal, will be small because of the exponential decay factor included in the norm (4.29) (see (4.88) below) and the one of $(\mathcal{L} \ell)_{\emptyset A^{\prime}}$ will be small because our inductive bound on $\|\ell\|_{\lambda}$ is smaller than our bound on $\|s\|_{\lambda}$, see (4.46 4.48). For the linear terms in (4.44), the norm of $\sigma_{\mathcal{D}^{\prime}}^{1}\left(1-\chi_{\mathcal{D}^{\prime}}\right) \delta_{A^{\prime}, \emptyset}$ will be small because our definition (4.20) of $r_{z^{\prime}}$ (see (4.90) below) and the one of $\mathcal{L} \ell$ will be small because the operator $\mathcal{L}_{i}$ is a contraction for fixed $i$, see (4.76) below, and because, unlike $s, \ell$ occurs only at times where $N_{z} \neq 0$, and this effectively controls the sum over times in (3.15) that made $s$ a relevant variable, see (4.89) below. This contraction also implies that the linear term in (4.33) contracts since there is no sum over times in (4.14).

Let us now bound the nonlinear term $\mathcal{N}^{i}, i=1,2$ in (4.30, 4.33). This is given by a sum of the products $\Pi$ in (4.31) localized in (4.38). To simplify the notation, we 
consider here only $\tilde{\mathcal{D}}_{i}$ being a single set and not a pair of such sets, as they occur for $\alpha=2,3$, but the Lemma below extends to those cases.

Lemma 4.9. $\Pi$ defined in (4.37), (4.38) satisfies:

$$
\|\Pi\|_{2 \lambda} \leq C(L) \prod_{i}\left\|\tilde{B}_{t_{i}}^{\alpha_{i}}\right\|_{\frac{\lambda}{3}}
$$

where $\tilde{B}_{t_{i}}^{\alpha_{i}}$ is defined in (4.35) (see the discussion leading to (4.37)), and satisfies:

$$
\left\|\tilde{B}_{t_{i}}^{\alpha_{i}}\right\|_{\frac{\lambda}{3}} \leq C\left\|B_{t_{i}}^{\alpha_{i}}\right\|_{\lambda}
$$

Proof. Recall that, from (4.29),

$$
\|\Pi\|_{2 \lambda}=\sup _{v^{\prime}} \sum_{\mathcal{D}^{\prime}, A^{\prime}, u^{\prime}}\left\|\Pi_{t^{\prime}, \mathcal{D}^{\prime}, A^{\prime}}\right\|_{u^{\prime}, v^{\prime}} e^{-N_{t^{\prime}}^{\prime}\left(\mathcal{D}^{\prime}\right)} e^{2 \lambda l\left(\mathcal{D}^{\prime}, A^{\prime}, t^{\prime}, u^{\prime}, v^{\prime}\right)}
$$

We have from (4.37,4.39)

$$
\left\|\Pi_{t^{\prime}, \mathcal{D}^{\prime}, A^{\prime}}\right\|_{u^{\prime}, v^{\prime}} \leq L^{d-1} \sup _{u_{1} \in L \mathbf{u}^{\prime}, v_{N} \in L \mathbf{v}^{\prime}} \sum_{v_{1}, u_{2}, \ldots, u_{N}} \sum_{\left\{\tilde{\mathcal{D}}_{i}, \tilde{A}_{i}\right\}} \prod_{i=1}^{N}\left\|\tilde{B}_{t_{i}, \tilde{\mathcal{D}}_{i}, \tilde{A}_{i}}^{\alpha_{i}}\right\|_{u_{i} v_{i}} .
$$

From (4.24) we have $N_{z^{\prime}}^{\prime} \geq \tilde{N}_{z^{\prime}}-1$. Since, see (4.39), $\mathcal{D}^{\prime}=\left[\cup_{i} \tilde{\mathcal{D}}_{t_{i}}\right]$ (leaving out the index $t^{\prime}$ in $D^{\prime}$ ) we deduce, using (4.23) and (4.28),

$$
-N_{t^{\prime}}^{\prime}\left(D^{\prime}\right) \leq-\sum_{i} N_{t_{i}}\left(\tilde{\mathcal{D}}_{t_{i}}\right)+\left|D^{\prime}\right|
$$

For the geometrical factor (4.27), we have

$$
l\left(D^{\prime}, A^{\prime}, t^{\prime}, u^{\prime}, v^{\prime}\right) \leq \sum_{i}\left(\frac{c}{L} l\left(\tilde{\mathcal{D}}_{i}, \tilde{A}_{i}, t_{i}, u_{i}, v_{i}\right)+c\right)
$$

Indeed, this inequality obviously holds both for the graph length and for the diameter terms in (4.27), since the blocking operation (4.5) effectively scales space-time distances by a factor at least $L^{-1}$ (except for small sets, hence the $+c$ term in (4.67)) and since $u_{1} \in L \mathbf{u}^{\prime}, v_{N} \in L \mathbf{v}^{\prime}$. Since the LHS is also larger than $\left|D^{\prime}\right|$ (because $\tau\left(D^{\prime}\right) \geq\left|D^{\prime}\right|$ ), we deduce:

$$
\begin{aligned}
& 2 \lambda l\left(D^{\prime}, A^{\prime}, t^{\prime}, u^{\prime}, v^{\prime}\right) \leq(2 \lambda+1) l\left(D^{\prime}, A^{\prime}, t^{\prime}, u^{\prime}, v^{\prime}\right)-\left|D^{\prime}\right| \\
\leq & \sum_{i}(2 \lambda+1) \frac{c}{L} l\left(\tilde{\mathcal{D}}_{i}, \tilde{A}_{i}, t_{i}, u_{i}, v_{i}\right)+(2 \lambda+1) L^{2} c-\left|D^{\prime}\right| .
\end{aligned}
$$

Taking $L(\lambda)=3(2+1 / \lambda) c$ and inserting (4.65), (4.66) and (4.68) in (4.64), the claim (4.62) follows.

For (4.63), we recall that $\tilde{\mathcal{D}}_{i}=\mathcal{D}_{i} \cap L D^{\prime}$ and that, for $z^{\prime} \notin D^{\prime}, \tilde{N}_{z^{\prime}}<\frac{\lambda}{3}$. These imply

$$
-N_{t_{i}}\left(\tilde{\mathcal{D}}_{i}\right) \leq-N_{t_{i}}\left(\mathcal{D}_{i}\right)+\frac{\lambda}{3}\left|\mathcal{D}_{i} \backslash L D^{\prime}\right| \leq-N_{t_{i}}\left(\mathcal{D}_{i}\right)+\frac{\lambda}{3}\left|\mathcal{D}_{i}\right|
$$

As for the geometric factors, using (4.36), we get:

$$
\tau\left(\mathcal{D}_{i}, u, v\right) \geq \frac{1}{2}\left(\tau\left(\tilde{\mathcal{D}}_{i}, u, v\right)+d\left(\left(\tilde{A}_{i} \backslash A_{i}\right) \cup(u, t) \cup(v, t)\right),\right.
$$

which we rewrite as

$$
\tau\left(\tilde{\mathcal{D}}_{i}, u, v\right) \leq 2 \tau\left(\mathcal{D}_{i}, u, v\right)-d\left(\left(\tilde{A}_{i} \backslash A_{i}\right) \cup(u, t) \cup(v, t)\right),
$$


and combine with

$$
d\left(\tilde{A}_{i} \cup(u, t) \cup(v, t)\right) \leq d\left(A_{i} \cup(u, t) \cup(v, t)\right)+d\left(\left(\tilde{A}_{i} \backslash A_{i}\right) \cup(u, t) \cup(v, t)\right)
$$

in order to derive, see (4.27),

$$
l\left(\tilde{\mathcal{D}}_{i}, \tilde{A}_{i}, t_{i}, u, v\right) \leq 2 l\left(\mathcal{D}_{i}, A_{i}, t_{i}, u, v\right) \leq 3 l\left(\mathcal{D}_{i}, A_{i}, t_{i}, u, v\right)-\left|\mathcal{D}_{i}\right| .
$$

where in the last inequality we used $l\left(\mathcal{D}_{i}, A_{i}, t_{i}, u, v\right) \geq\left|\mathcal{D}_{i}\right|$. Insert (4.69), (4.70) in (4.29), (4.35), and the claim (4.63) follows.

This Lemma allows us to bound the nonlinear terms $\mathcal{N}^{i}, i=1,2$, by inspection using the inductive bounds (4.46)-(4.50), the bound (4.57) for $T$ and the fact that $\mathcal{N}^{i}$ are sums of $\Pi$ 's with an $L$ dependent number of terms.

Let us start with the nonlinear term $\mathcal{N}^{1}$ corresponding to $b$, setting $t^{\prime}=0$ (see Remark 4.8). The largest contribution is linear in $B$ i.e. coming from the term

$$
L^{d-1} \sum_{t} \mathcal{S}_{L} T^{L^{2}-t-2} B_{t} T^{t}
$$

whose norm is bounded by $C(L) \epsilon_{n}^{1-\gamma / 2}$ (using (4.49)). Terms involving $b \nabla B$ are bounded, using inductively Proposition 4.1 , by $C(L) \epsilon_{n}^{3 / 2-3 \gamma}$. Other terms are smaller and we get from the Lemma:

$$
\left\|\mathcal{N}^{1}\right\|_{2 \lambda} \leq \frac{1}{8} \epsilon_{n+1}^{1-\gamma}
$$

for $\epsilon$ small.

For $\mathcal{N}^{2}$, corresponding to $B$, the largest terms are the quadratic ones

$$
L^{d-1} \mathcal{S}_{L} T^{\tau_{1}} b_{\tau_{2}+\tau_{3}+1} \nabla \cdot T^{\tau_{2}} b_{\tau_{3}} T^{\tau_{3}}
$$

with $\tau_{1}+\tau_{2}+\tau_{3}+1=2 L^{2}-1, \tau_{2} \neq 0, \tau_{3} \leq L^{2}-1 \tau_{2}+\tau_{3}+1 \geq L^{2}$, and

$$
L^{d-1} \mathcal{S}_{L} T^{L^{2}-1} b_{L^{2}} \nabla \cdot B_{L^{2}-2} T^{L^{2}-2}
$$

The norms of these are bounded by $C(L) \epsilon_{n}^{2(1-3 \gamma)}$ and $C(L) \epsilon_{n}^{(1-3 \gamma)+1 / 2}$ respectively; other terms, e.g. $L^{d-1} \mathcal{S}_{L} T^{L^{2}-2} B_{L^{2}} \nabla \cdot B_{L^{2}-2} T^{L^{2}-2}$ are of order $C(L) \epsilon_{n}^{(1-\gamma / 2)+1 / 2}$, and so

$$
\left\|\mathcal{N}^{2}\right\|_{2 \lambda} \leq \frac{1}{2} \epsilon_{n+1}^{(1-\gamma / 2)}
$$

since $\gamma<1 / 12$ and $\epsilon$ is small.

Finally, to estimate $\nabla \cdot \mathcal{N}^{2}$ for (4.50) the leading term is of the form $L^{d-1} \mathcal{S}_{L} \nabla$. $b_{2 L^{2}-1} \nabla \cdot T^{2 L^{2}-t-2} b_{t} T^{t}$, for $t \leq L^{2}-1$; indeed, if $\nabla$ acts on a $T$, we can use (4.57), which gives a smaller contribution; we use the bound (4.51) for $\nabla \cdot b_{2 L^{2}}$ to get

$$
\left\|\nabla \cdot \mathcal{N}^{2}\right\|_{2 \lambda} \leq C(L) \epsilon \epsilon_{n}^{1-6 \gamma} \leq \frac{1}{2} \epsilon_{n+1}^{1 / 2} .
$$

Since $\gamma<\frac{1}{12}$ and $\epsilon$ is small.

To bound $\nabla \cdot \mathcal{L} B^{2}$, we use inductively (4.49) and the fact that, if we apply $\nabla$ to (4.14), it acts on a $T$ and we can use (4.57) to bound it. We get then

$$
\left\|\nabla \cdot \mathcal{L} B^{2}\right\|_{2 \lambda} \leq \frac{1}{2} \epsilon_{n+1}^{1 / 2} .
$$

This and (4.73) finishes the iteration of (4.50). 
We will now consider the linear RG contributions to (4.42)-(4.45). To deal with the $\sigma$ of (4.6) and $\mathcal{L} \ell$ of (4.41), we need a fundamental bound on the operator $\mathcal{L}$ :

Lemma 4.10. Let $\beta$ denote $s$ or $\ell$. For $t \in I_{t^{\prime}}$ define

$$
c_{D^{\prime} A^{\prime}}^{t}:=e^{\sum_{s \neq t} N_{s}\left(L D^{\prime}\right)} \sum_{\tilde{\mathcal{D}}, \tilde{A}} \mathcal{L}_{i} \tilde{\beta}_{t, \tilde{\mathcal{D}}, \tilde{A}} .
$$

where $i=t-L^{2} t^{\prime},[\tilde{\mathcal{D}}]=D^{\prime}$ and $\tilde{A} \in \mathcal{A}\left(A^{\prime}\right)($ for $\beta=s)$ or $[\tilde{A}]=A^{\prime}($ for $\beta=\ell)$. Then,

$$
\left\|c^{t}\right\|_{2 \lambda} \leq C L^{-1}\left\|\tilde{\beta}_{t}\right\|_{\frac{\lambda}{3}},
$$

Proof. Write, using (3.15) and $\left|u^{\prime}-\frac{u}{L}\right| \leq\left|x^{\prime}-\frac{x}{L}\right|+2,\left|v^{\prime}-\frac{v}{L}\right| \leq\left|y^{\prime}-\frac{y}{L}\right|+2$, for $x^{\prime} \in \mathbf{u}^{\prime}, x \in \mathbf{u}, y^{\prime} \in \mathbf{v}^{\prime}, y \in \mathbf{v}$,

$$
\left\|\mathcal{L}_{i} \tilde{\beta}_{t, \tilde{\mathcal{D}}, \tilde{A}}\right\|_{u^{\prime}, v^{\prime}} \leq C L^{d-1} \sum_{u, v}\left\|\beta_{t, \tilde{\mathcal{D}}, \tilde{A}}\right\|_{u, v} e^{-\frac{k}{L}\left(\left|L u^{\prime}-u\right|+\left|L v^{\prime}-v\right|\right)} S_{i}\left(u, v, u^{\prime}, v^{\prime}\right),
$$

with

$$
S_{i}\left(u, v, u^{\prime}, v^{\prime}\right)=\sup _{\substack{x^{\prime} \in \mathbf{u}^{\prime} \\ y^{\prime} \in \mathbf{v}^{\prime}}} \int_{\mathbf{u}} d x \tilde{T}^{L^{2}-i-1}\left(L x^{\prime}-x\right) \int_{\mathbf{v}} d y \tilde{T}^{i}\left(y-L y^{\prime}\right)
$$

and $\tilde{T}^{t}(x)=T^{t}(x) \exp \left(\frac{k|x|}{L}\right)$. As in (4.66), we get

$$
-N_{t^{\prime}}^{\prime}\left(D^{\prime}\right) \leq-N_{t}(\tilde{\mathcal{D}})-\sum_{s \neq t} N_{s}\left(L D^{\prime}\right)+\left|D^{\prime}\right|
$$

As for the geometric factors, since $A^{\prime} \subset[\tilde{A}]$ for all $\tilde{A} \in \mathcal{A}\left(A^{\prime}\right)$ we have, as in (4.67),

$$
l\left(D^{\prime}, A^{\prime}, t^{\prime}, u^{\prime}, v^{\prime}\right) \leq \frac{c}{L}\left(l(\tilde{\mathcal{D}}, \tilde{A}, t, u, v)+\left|L u^{\prime}-u\right|+\left|L v^{\prime}-v\right|\right)+c .
$$

Since the LHS is also larger than $\frac{1}{2}\left(\left|D^{\prime}\right|+\left|u^{\prime}-v^{\prime}\right|\right)$ we get as in (4.68)

$$
\begin{aligned}
2 \lambda l\left(D^{\prime}, A^{\prime}, t^{\prime}, u^{\prime}, v^{\prime}\right) \leq & (\lambda+1) \frac{2 c}{L}\left(l(\tilde{\mathcal{D}}, \tilde{A}, t, u, v)+\left|L u^{\prime}-u\right|+\left|L v^{\prime}-v\right|\right) \\
& +(\lambda+1) 2 c-\left(\left|D^{\prime}\right|+\left|u^{\prime}-v^{\prime}\right|\right) .
\end{aligned}
$$

We take

$$
k=(\lambda+1) 2 c
$$

and so we have $\frac{k}{L}=(\lambda+1) \frac{2 c}{L} \leq \lambda / 6$ for $L$ large. Using $l(\tilde{\mathcal{D}}, \tilde{A}, t, u, v) \geq|u-v|$, we get:

$$
\begin{aligned}
2 \lambda l\left(D^{\prime}, A^{\prime}, t^{\prime}, u^{\prime}, v^{\prime}\right) \leq & \frac{\lambda}{3} l(\tilde{\mathcal{D}}, \tilde{A}, t, u, v)+\frac{k}{L}\left(\left|L u^{\prime}-u\right|+\left|L v^{\prime}-v\right|\right) \\
& +k-\left(\left|D^{\prime}\right|+\left|u^{\prime}-v^{\prime}\right|\right)-\frac{\lambda}{6}|u-v| .
\end{aligned}
$$

Then, inserting (4.79) and (4.81) in (4.75), (4.77), we get:

$$
\left\|c^{t}\right\|_{2 \lambda} \leq C L^{d-1}\left\|\tilde{\beta}_{t}\right\|_{\frac{\lambda}{3}} \sup _{v^{\prime}} \sum_{u^{\prime}, u, v} e^{-\left|u^{\prime}-v^{\prime}\right|} e^{-\frac{\lambda}{6}|u-v|} S_{i}\left(u, v, u^{\prime}, v^{\prime}\right) .
$$


Note that we have $\frac{k}{L} \leq \frac{\lambda}{2}$ and that $C(k)$ in (4.60) is $L$-independent, i.e. we may write $C(k)=C$ in (4.60). Then, use (4.60) to bound, for any $v^{\prime}$,

$$
\sum_{u, v} e^{-\frac{\lambda}{6}|u-v|} S_{i}\left(u, v, u^{\prime}, v^{\prime}\right) \leq C \min \left(i^{-\frac{d}{2}},\left(L^{2}-i-1\right)^{-\frac{d}{2}}\right) \leq C^{\prime} L^{-d}
$$

where the $L^{\infty}$ bound is used in (4.78) for the integral corresponding to the minimum in (4.83); the $L^{1}$ bound and the factor $e^{-\frac{\lambda}{6}|u-v|}$ control the remaining integral and the sum over $u, v$. Finally, use $e^{-\left|u^{\prime}-v^{\prime}\right|}$ to control the sum over $u^{\prime}$ in (4.82).

Combining (4.82) and (4.83) we get the claim (4.76).

Let us look at the consequences of Lemma 4.10. The simplest one is gotten by taking $\beta=s$ in (4.75). Then summing ( 4.76) over the times and using (4.63) yields, for $\sigma=\mathcal{L} s$,

$$
\|\sigma\|_{2 \lambda} \leq C L\|\tilde{s}\|_{\frac{\lambda}{3}} \leq C L\|s\|_{\lambda}
$$

which just expresses the fact that the linear RG has an expanding bound.

Next, recall we have two decompositions for $\sigma$ : in (4.43) $\sigma=\sigma^{1}+\sigma^{2}$ where $\sigma^{1}=\mathcal{L} s^{\text {loc; }}$; but we can also write, as in (4.16),$\sigma=\sigma^{\text {loc }}+\sigma^{\text {nloc }}$. Thus the local part of $\sigma^{2}$ equals the local part of $\mathcal{L} s^{\text {nloc }}$, which exists because of the "blocking" in (4.6). Lemma 4.10 and (4.63) then gives

$$
\left\|\left(\sigma^{2}\right)^{\mathrm{loc}}\right\|_{2 \lambda} \leq C L\left\|s^{\mathrm{nloc}}\right\|_{\lambda} \leq C L \epsilon_{n}^{1-2 \gamma} \leq \frac{1}{8} \epsilon_{n+1}^{1-3 \gamma}
$$

using (4.47) and $\epsilon$ small in the last two inequalities.

Since from (4.20) and the definition of $\chi$ in (4.42), (4.20), we have

$$
\left\|\sigma^{1} \chi\right\|_{\lambda} \leq \frac{1}{8} \epsilon_{n+1}^{1-3 \gamma}
$$

we conclude

$$
\left\|\mathbb{E}^{\perp}\left(\sigma^{1} \chi+\left(\sigma^{2}\right)^{\mathrm{loc}}\right)\right\|_{\lambda} \leq \frac{1}{2} \epsilon_{n+1}^{1-3 \gamma}
$$

since the norm of $\mathbb{E}^{\perp}$ is bounded by 2 .

Next, apply Lemma 4.10 to $\beta=s 1_{d(A)>\frac{L}{4}}$. Then, since $l$ in (4.27) is larger than $d(A)$

$$
\|\beta\|_{\frac{\lambda}{3}} \leq e^{-\frac{\lambda L}{6}}\left\|s^{\text {nloc }}\right\|_{\lambda} .
$$

Summing over the times $t$, we get from (4.76), the definition (4.6) and (4.47) used inductively,

$$
\left\|\sigma^{\text {nloc }}\right\|_{2 \lambda} \leq C L e^{-\frac{\lambda L}{6}}\left\|s^{\text {nloc }}\right\|_{\lambda} \leq \frac{1}{4} \epsilon_{n+1}^{1-2 \gamma},
$$

which means that the non local part is irrelevant under the linear RG.

Next apply (4.76) to $\beta=\ell$. Given $D^{\prime}$, let

$$
T\left(D^{\prime}\right)=\left\{s \in I_{t^{\prime}} \mid N_{s}\left(L D^{\prime}\right) \neq 0\right\} .
$$

Thus, for $s \in T\left(D^{\prime}\right), N_{s}\left(L D^{\prime}\right) \geq \lambda / 3$ and so

$$
\sum_{t \in T\left(D^{\prime}\right)} e^{-\sum_{s \neq t} N_{s}\left(L D^{\prime}\right)} \leq\left|T\left(D^{\prime}\right)\right| e^{-\frac{\lambda}{3}\left(\left|T\left(D^{\prime}\right)\right|-1\right)} \leq C .
$$


Now, writing (4.75) with $\beta=\ell$ as $\sum_{\tilde{\mathcal{D}}, \tilde{A}} \mathcal{L}_{i} \tilde{l}_{t, \tilde{\mathcal{D}}, \tilde{A}}=e^{-\sum_{s \neq t} N_{s}\left(L D^{\prime}\right)} c_{D^{\prime} A^{\prime}}^{t}$, using (4.41), and combining the last bound (to control the sum over times) with (4.76), (4.63) and the inductive bound (4.48), we get

$$
\left\|(\mathcal{L} \ell)_{t^{\prime}}\right\|_{2 \lambda} \leq C L^{-1} \epsilon_{n}^{1-\gamma} \leq \frac{1}{8} \epsilon_{n+1}^{1-\gamma}
$$

for $L$ large. Eq. (4.89) is also a bound for the $\|\cdot\|_{\lambda}$ norm of the third term on the RHS of (4.42). Inserting (4.87), (4.89) and (4.71) in (4.42), and bounding the norm of $\mathbb{E}^{\perp}$ by 2 , the induction step for (4.46) follows; inserting (4.88), (4.89) and (4.71) in (4.42), we get the induction step for (4.47).

Finally, to bound (4.44), use (4.89) and (4.71) for the first and third terms on the RHS. For the second one, we use $r_{z^{\prime}}=\left(n_{0}+n\right) \log L$ and the definition (4.29) to bound its norm by

$$
C(L) L^{-n-n_{0}} \epsilon_{n}^{1-3 \gamma} \leq \frac{1}{8} \epsilon_{n+1}^{1-\gamma}
$$

since we have taken, see (4.21), $L^{-n_{0}}=\epsilon^{3 \gamma}$, and we use $\epsilon^{\gamma}$ to control $8 C(L)$. Finally, bound the norm of $E^{\perp}$ by two.

To finish the inductive step in the proof of Proposition 4.1 we need to consider the linear RG (4.14) for $B$. The operator $\mathcal{L}$ is bounded as in (4.76), and since, in (4.14), there is no sum over times, we get $\left(B^{2}=B\right)$

$$
\left\|\mathcal{L} B^{2}\right\|_{2 \lambda} \leq C L^{-1}\left\|B^{2}\right\|_{\lambda}
$$

Combining this with (4.33), (4.72), and using inductively (4.49), we get (4.49) on scale $n+1$.

4.11. Proof of Proposition 4.4. It suffices to consider $t^{\prime}=0$ (see Remark 4.8) and so we will suppress the argument $t^{\prime}$. In Section 4.10. we have shown, see (4.42, 4.20),

$$
s_{u^{\prime}}^{\prime}=\mathbb{E}^{\perp} \sigma_{u^{\prime}}^{1} \chi+\rho
$$

with

$$
\chi=\mathbb{1}\left(\left\|\sigma_{u^{\prime}}^{1}\right\|_{\lambda} \leq \frac{1}{8} \epsilon_{n}^{1-3 \gamma}\right)
$$

where, by the second inequality in (4.85), (4.88), $\sigma^{2}=\left(\sigma^{2}\right)^{l o c}+\sigma^{n l o c}$, (4.89) and (4.71),

$$
\|\rho\|_{2 \lambda} \leq C L \epsilon_{n+1}^{1-2 \gamma} \text {. }
$$

From (3.15), (4.17), we have

$$
\sigma_{u^{\prime}}^{1}=\left(\mathcal{L} s^{l o c}\right)_{u^{\prime}}=\sum_{t=0}^{L^{2}-1} \sum_{u \in L u^{\prime}} \mathcal{L}_{i} s_{t, u} .
$$

By Schwarz' inequality,

$$
\mathbb{E} e^{\left(s_{u^{\prime}}^{\prime}, f\right)} \leq\left(\mathbb{E} e^{\mathbb{E}^{\perp}\left(\sigma_{u^{\prime}}^{1} \chi, 2 f\right)}\right)^{\frac{1}{2}}\left(\mathbb{E} e^{\left(\rho_{u^{\prime}}, 2 f\right)}\right)^{\frac{1}{2}}
$$

Let us first discuss the second factor on the RHS of (4.95). Write, using (4.53), $\left(\rho_{u^{\prime}}, 2 f\right)=2 \int d x d y \rho_{u^{\prime}}(x, y) f(x, y) \exp \left(-2 \lambda \tau\left(u^{\prime}, x, y\right)\right) \exp \left(2 \lambda \tau\left(u^{\prime}, x, y\right)\right)$. Using, for $x \in \mathbf{u}, y \in \mathbf{v}, \tau\left(u^{\prime}, x, y\right) \leq \tau(u \cup v)+d\left(u^{\prime} \cup u \cup v\right)$ (since the right hand side is 
greater than the sum of the length of path joining $u$ and $v$ and one joining $u^{\prime}$ and $u$ or $v$ ), and using the definition of the norms (4.29) and (4.54), we get:

$$
\left|\left(\rho_{u^{\prime}}, 2 f\right)\right| \leq C\|\rho\|_{2 \lambda}\|f\|_{u^{\prime}} \leq C L \epsilon_{n+1}^{1-2 \gamma}\|f\|_{u^{\prime}}
$$

using (4.93) in the last inequality. Let first $f$ be such that the right-hand side of (4.96) is less than 1 . Using $\mathbb{E}\left(\rho_{u^{\prime}}, 2 f\right)=0$ and the inequality $\left|e^{x}-1-x\right| \leq x^{2}$ for $|x|<1$ we get, using (4.96),

$$
\mathbb{E} e^{\left(\rho_{u^{\prime}}, 2 f\right)} \leq 1+\mathbb{E}\left(\rho_{u^{\prime}}, 2 f\right)^{2} \leq \exp \left(C L^{2} \epsilon_{n+1}^{2-4 \gamma}\|f\|_{u^{\prime}}^{2}\right) \leq \exp \left(\delta_{n+1}\|f\|_{u^{\prime}}^{2}\right),
$$

for $\epsilon$ small, with $\delta_{n+1}=L^{-(n+1) \gamma} \epsilon_{n+1}^{2-6 \gamma}$.

On the other hand, if $f$ is such that the right hand side of (4.96) is larger than 1 , we can bound:

$$
\mathbb{E} \exp \left(\rho_{u^{\prime}}, 2 f\right) \leq \mathbb{E} \exp \left|\left(\rho_{u^{\prime}}, 2 f\right)\right|,
$$

and use (4.96) and $x<x^{2}$ for $x>1$ to get (4.97) again.

Consider then the first factor on the RHS of (4.95). We have, by (4.53), (4.6), (3.15),

$$
\left(\sigma_{u^{\prime}}^{1}, f\right)=\sum_{t=0}^{L^{2}-1} \sum_{u \in L u^{\prime}}\left(s_{t, u}, f_{t}\right),
$$

with

$$
f_{t}(x, y):=L^{d-1} \int d x^{\prime} d y^{\prime} f\left(x^{\prime}, y^{\prime}\right) T^{L^{2}-t-1}\left(L x^{\prime}-x\right) T^{t}\left(y-L y^{\prime}\right) .
$$

Lemma 4.11. Let $u \in L \mathbf{u}^{\prime}$. Then, $\forall f: \mathbb{R}^{d} \times \mathbb{R}^{d} \rightarrow \mathbb{R}$ measurable,

$$
\begin{aligned}
\left\|f_{t}\right\|_{u} & \leq C L^{-1}(t+1)^{-d / 2}\|f\|_{u^{\prime}} \\
\sum_{u \in L \mathbf{u}^{\prime}}\left\|f_{t}\right\|_{u} & \leq C L^{-1}\|f\|_{u^{\prime}}
\end{aligned}
$$

Proof. By (4.54) and (4.99) we get

$$
\left\|f_{t}\right\|_{u} \leq L^{d-1} \int d x d y d x^{\prime} d y^{\prime}\left|f\left(x^{\prime}, y^{\prime}\right)\right| T^{L^{2}-t-1}\left(L x^{\prime}-x\right) T^{t}\left(y-L y^{\prime}\right) e^{-2 \lambda \tau(u, x, y)} .
$$

Now use the triangle inequality to get:

$$
\left.\tau\left(u^{\prime}, x^{\prime}, y^{\prime}\right)\right) \leq \frac{c}{L}\left(\tau(u, x, y)+\left|L x_{i}^{\prime}-x\right|+\left|y-L y^{\prime}\right|\right)+c,
$$

which gives, if $\frac{c}{L}<1 / 4$

$$
\left\|f_{t}\right\|_{u} \leq C L^{d-1} \int d x^{\prime} d y^{\prime}\left|f\left(x^{\prime}, y^{\prime}\right)\right| \exp \left(-2 \lambda \tau\left(u^{\prime}, x^{\prime}, y^{\prime}\right)\right) I\left(x^{\prime}, y^{\prime}, u\right)
$$

with

$$
I\left(x^{\prime}, y^{\prime}, u\right)=\int d x d y \tilde{T}^{L^{2}-t-1}\left(L x^{\prime}-x\right) \tilde{T}^{t}\left(y-L y^{\prime}\right) \exp (-\lambda \tau(u, x, y))
$$

and, as before, $\tilde{T}^{t}(x)=T^{t}(x) \exp \left(\frac{2 c \lambda|x|}{L}\right)$. By symmetry, we may suppose $t \leq \frac{L^{2}}{2}$. Since $k=2 c \lambda$, and $\frac{c}{L}<1 / 4, \frac{k}{L} \leq \frac{\lambda}{2}$, and we may use (4.60), with $C(k) L$-independent. The sup norm bound in (4.60) yields

$$
I\left(x^{\prime}, y^{\prime}, u\right) \leq C L^{-d}(t+1)^{-d / 2} \int d x d y \exp (-\lambda \tau(u, x, y))
$$




$$
\leq C L^{-d}(t+1)^{-d / 2} \text {. }
$$

Combined with (4.102) we obtain the first claim (4.100).

For the second claim, use (4.58) to get

$$
\exp \left(-\frac{\lambda}{2} \tau(u, x, y)\right) \tilde{T}^{t}\left(y-L y^{\prime}\right) \leq C(t+1)^{-d / 2} \exp \left(-c \lambda \frac{\left|u-L y^{\prime}\right|}{\sqrt{t+1}}\right),
$$

where $C$ and $c$ are $L$-independent. Then, using the sup norm bound in (4.60) to get $\tilde{T}^{L^{2}-t-1}\left(L x^{\prime}-x\right) \leq C L^{-d}$, for $t \leq \frac{L^{2}}{2}$, and the bounds

$$
\begin{gathered}
\int d x d y \exp \left(-\frac{\lambda}{2} \tau(u, x, y)\right) \leq C, \\
\sum_{u \in \mathbb{Z}^{d}}(t+1)^{-d / 2} \exp \left(-c \lambda \frac{\left|u-L y^{\prime}\right|}{\sqrt{t+1}}\right) \leq C,
\end{gathered}
$$

we get:

$$
\sum_{u \in \mathbb{Z}^{d}} I\left(x^{\prime}, y^{\prime}, u\right) \leq C L^{-d}
$$

whereby, using (4.102), (4.101) follows.

The lemma allows us to prove

\section{Lemma 4.12.}

$$
\mathbb{E} e^{\left(\sigma_{u^{\prime}}^{1}, 2 f\right)} \leq e^{L^{-\gamma} \delta_{n+1}\|f\|_{u^{\prime}}^{2}} .
$$

Proof. As in (4.96), we have $\left|\left(\sigma_{u^{\prime}}^{1}, 2 f\right)\right| \leq C\left\|\sigma^{1}\right\|_{2 \lambda}\|f\|_{u^{\prime}}$. We obtain, as in (4.84), taking $\beta=s^{l o c}$, instead of $s$ in (4.75), and using inductively Proposition 4.1,

$$
\left\|\sigma^{1}\right\|_{2 \lambda} \leq C L \epsilon_{n}^{1-3 \gamma} \text {. }
$$

We then get that

$$
\left|\left(\sigma_{u^{\prime}}^{1}, 2 f\right)\right| \leq C L \epsilon_{n}^{1-3 \gamma}\|f\|_{u^{\prime}}
$$

Consider first $f$ such that the RHS of (4.109) is less than 1 . Using $\mathbb{E}\left(\sigma_{u^{\prime}}^{1}, 2 f\right)=0$ and the same argument as before (4.97), we get

$$
\mathbb{E} e^{\left(\sigma_{u^{\prime}}^{1}, 2 f\right)} \leq 1+\mathbb{E}\left(\sigma_{u^{\prime}}^{1}, 2 f\right)^{2} .
$$

By (4.98),

$$
\mathbb{E}\left(\sigma_{u^{\prime}}^{1}, f\right)^{2}=\sum_{t_{1}, t_{2}=0}^{L^{2}-1} \sum_{u_{1}, u_{2} \in L u^{\prime}} \mathbb{E}\left(s_{t_{1}, u_{1}}, f_{t_{1}}\right)\left(s_{t_{2}, u_{2}}, f_{t_{2}}\right)
$$

Denote $z_{i}=\left(u_{i}, t_{i}\right)$ and distinguish between the sum over $\left|z_{1}-z_{2}\right| \leq L^{\beta}$ and $\left|z_{1}-z_{2}\right| \geq$ $L^{\beta}$, where $\beta$ will be chosen below.

For the first sum, we use Schwarz inequality on $\mathbb{E}\left(s_{t_{1}, u_{1}}, f_{t_{1}}\right)\left(s_{t_{2}, u_{2}}, f_{t_{2}}\right)$ and then Schwarz inequality again for the sum over $z_{1}, z_{2}$ with the constraint $\left|z_{1}-z_{2}\right| \leq L^{\beta}$, and (4.56), to bound that sum by $C L^{\gamma} \delta_{n} \sum_{u, t}\left\|f_{t}\right\|_{u}^{2}$, if we choose $(d+1) \beta \leq \gamma$.

For $\left|z_{1}-z_{2}\right| \geq L^{\beta}$ we apply (2.3) with $F_{i}=\left(s_{t_{i}, u_{i}}, f_{t_{i}}\right)$. The supports of these functions are, in the original lattice, at a distance at least $L^{\beta+n}$ and their size is 
bounded by $C L^{(2+d) n}$; we have, $\left|\left(s_{t_{i}, u_{i}}, f_{t_{i}}\right)\right| \leq C\left\|s_{t_{i}}\right\|_{2 \lambda}\left\|f_{t_{i}}\right\|_{u_{i}} \leq C L \epsilon_{n-1}^{1-3 \gamma} \sup _{u, t}\left\|f_{t}\right\|_{u}$, where, in the last inequality, we use (4.92) (with prime referring to scale $n$ ), (4.93), (4.108) (on scale $n-1$ ) and where the supremum is taken over $u, t$ in (4.111). This gives a bound on $\left\|F_{i}\right\|_{\infty}$ and we have $\mathbb{E}\left(F_{i}\right)=0$. By (2.3) the sum of the absolute value of those terms is bounded by

$$
C L^{(2+d) n} L^{2} \epsilon_{n-1}^{2-6 \gamma} \exp \left(-c L^{\beta+n}\right) \sup _{u, t}\left\|f_{t}\right\|_{u}^{2}
$$

Since for $L$ large $C L^{(2+d) n} L^{2} \exp \left(-c L^{\beta+n}\right) \leq L^{-2} L^{-n \gamma}$ and $\epsilon_{n-1}^{2-6 \gamma} L^{-2} L^{-n \gamma} \leq \delta_{n}$, we conclude that:

$$
\mathbb{E}\left(\sigma_{u^{\prime}}^{1}, 2 f\right)^{2} \leq C L^{\gamma} \delta_{n} \sum_{u, t}\left\|f_{t}\right\|_{u}^{2}
$$

By Lemma 4.11

$$
\sum_{u, t}\left\|f_{t}\right\|_{u}^{2} \leq C L^{-2}\|f\|_{u^{\prime}}^{2} \sum_{t \leq L^{2}}(t+1)^{-d / 2}
$$

Since the sum is bounded by $C \log L \leq L^{\gamma}$, for $d \geq 2$ we get, using (4.110), (4.112), in the case where $f$ is such that the RHS of (4.109) is less than 1 ,

$$
\mathbb{E} e^{\left(\sigma_{u^{\prime}}^{1}, 2 f\right)} \leq e^{C L^{-2+2 \gamma} \delta_{n}\|f\|_{u^{\prime}}^{2}} \leq e^{L^{-\gamma} \delta_{n+1}\|f\|_{u^{\prime}}^{2}} .
$$

Now, consider the case where $f$ is such that the RHS of (4.109) is larger than 1. Decompose $\mathbb{Z}^{d+1}$ into $C L^{\beta}$ sublattices $\mathbb{L}_{\ell}$ indexed by $\ell$, so that, if $z_{1}=\left(t_{1}, u_{1}\right), z_{2}=$ $\left(t_{2}, u_{2}\right)$ belong to the same sublattice, we have $\left|z_{1}-z_{2}\right| \geq L^{\beta}$, with again $(d+1) \beta \leq \gamma$. Using Hölder's inequality, we bound

$$
\mathbb{E} e^{\left(\sigma_{u^{\prime}}^{1}, 2 f\right)} \leq \prod_{\ell} \mathbb{E}\left(\exp \left(C L^{\gamma} \sum_{u, t}^{\ell}\left(s_{t, u}, f_{t}\right)\right)\right)^{\left(C L^{\gamma}\right)^{-1}}
$$

where $\sum^{\ell}$ means that the sum is restricted to $(u, t) \in\left(L u^{\prime} \times\left[0, L^{2}-1\right]\right) \cap \mathbb{L}_{\ell}$. Each of the factors in (4.115) is of the form $\mathbb{E}\left(\prod_{i=1}^{k} F_{i}\right)$ where the support of the functions $F_{i}$ are, in the original lattice, at a distance at least $L^{\beta+n}$ and their size is bounded by $C L^{(2+d) n}$. Hence, by (2.4),

$$
\mathbb{E} e^{\left(\sigma_{u^{\prime}}^{1}, 2 f\right)} \leq \prod_{\ell} \prod_{u, t}^{\ell}\left(\mathbb{E}\left(\exp \left(C L^{\gamma}\left(s_{t, u}, f_{t}\right)\right)\right)^{\left(C L^{\gamma}\right)^{-1}} \exp e^{-c L^{\beta+n}}\right)
$$

where the product $\prod^{\ell}$ runs over $(u, t) \in\left(L u^{\prime} \times\left[0, L^{2}-1\right]\right) \cap \mathbb{L}_{\ell}$ and where the factor $C L^{(2+d) n}$ is controlled by the exponential $e^{-c L^{\beta+n}}$.

Using inductively (4.55),

$$
\mathbb{E} e^{\left(\sigma_{u^{\prime}}^{1}, 2 f\right)} \leq \exp \left[C L^{\gamma} \delta_{n} \sum_{u, t}\left\|f_{t}\right\|_{u}^{2}+L^{2+d} e^{-c L^{\beta+n}}\right] .
$$

Use (4.113) and then use the fact that if $\|f\|_{u^{\prime}}$ is such that the RHS of (4.109) is larger than 1 , then $\delta_{n}\|f\|_{u^{\prime}}$ is larger than an inverse power of $L^{n}$, which shows that $L^{2+d} e^{-c L^{\beta+n}}$ is less than the first term, for $L$ large enough and all $n$; we end up again with the bound (4.114). The claim follows. 
Let us now bound the first factor in (4.95) using Lemma 4.12, First, by the exponential Tchebycheff inequality, (4.107) implies:

$$
\mathbb{P}\left(\left|\left(\sigma_{u^{\prime}}^{1}, 2 f\right)\right|>k\|f\|_{u^{\prime}}\right) \leq 2 \exp \left(-\frac{L^{\gamma} k^{2}}{4 \delta_{n+1}}\right)
$$

Let $a:=L^{-\frac{1}{2} \gamma} \delta_{n+1}^{\frac{1}{2}}\|f\|_{u^{\prime}}$. Then

$$
\begin{aligned}
\mathbb{E}\left(\left(\left|\left(\sigma_{u^{\prime}}^{1}, 2 f\right)\right|^{m}\right)\right. & \leq a^{m} \sum_{r=0}^{\infty}(r+1)^{m} \mathbb{P}\left(\left|\left(\sigma_{u^{\prime}}^{1}, 2 f\right)\right| \in[a r, a(r+1)]\right) \\
& \leq 2 a^{m} \sum_{r=0}^{\infty}(r+1)^{m} \exp \left(-\frac{r^{2}}{4}\right) \leq(C a)^{m}(m !)^{1 / 2} .
\end{aligned}
$$

Thus, since $\chi \leq 1$ and $\mathbb{E} \mathbb{E}^{\perp}=0$,

$$
\begin{aligned}
\mathbb{E} e^{\mathbb{E}^{\perp}\left(\sigma_{u^{\prime}}^{1} \chi, 2 f\right)} & \leq 1+\sum_{k=2}^{\infty} \frac{2^{k}}{k !} \max _{m \leq k}\left(\mathbb{E}\left(\left|\left(\sigma_{u^{\prime}}^{1}, 2 f\right)\right|^{m}\right)\left(\mathbb{E}\left(\left|\left(\sigma_{u^{\prime}}^{1}, 2 f\right)\right|\right)^{k-m}\right)\right. \\
& \leq 1+\sum_{k=2}^{\infty} \frac{(C a)^{k}}{k !}(k !)^{1 / 2} \leq e^{C a^{2}}=e^{C L^{-\gamma} \delta_{n+1}\|f\|_{u^{\prime}}^{2}} \leq=e^{\delta_{n+1}\|f\|_{u^{\prime}}^{2}} .
\end{aligned}
$$

Combining this with (4.97), (4.95), shows that (4.55) iterates.

4.12. Proof of Proposition 4.3. Let us first deduce the following Corollary from Proposition 4.4

Corollary 4.13. For all $z^{\prime} \in \mathbb{N} \times \mathbb{Z}^{d}$, and $n \geq 1$,

$$
\mathbb{P}\left(r_{z^{\prime}} \neq 0\right) \leq \exp \left(-c L^{(n+1) \gamma}\right)
$$

for some $c>0$.

Proof. From (4.20) we infer that if $r_{z^{\prime}} \neq 0$ then there exist $x, y, \zeta>0$, such that

$$
\left|\sigma_{u^{\prime}}^{1}(x, y)\right| \geq \zeta \epsilon_{n+1}^{1-3 \gamma} \exp \left(-3 \lambda \tau\left(u^{\prime}, x, y\right) / 2\right) .
$$

Indeed, otherwise, we could integrate the opposite bound over $x \in \mathbf{u}, y \in \mathbf{v}$, multiply the result by $\exp \left(\lambda \tau\left(u^{\prime}, u, v\right)\right)$, sum over $u, v$ and get, for $\zeta$ small enough, that $\left\|\sigma_{u^{\prime}}^{1}\right\|_{\lambda} \leq$ $\frac{1}{8} \epsilon_{n+1}^{1-3 \gamma}$, i.e. $r_{z^{\prime}}=0$

The bound (4.108) implies $\forall x, y$,

$$
\left|\sigma_{u^{\prime}}^{1}(x, y)\right| \leq C L \epsilon_{n}^{1-3 \gamma} e^{-2 \lambda \tau\left(u^{\prime}, x, y\right)} \leq \zeta \epsilon_{n+1}^{1-3 \gamma} \exp \left(-3 \lambda \tau\left(u^{\prime}, x, y\right) / 2\right),
$$

if $C e^{-\frac{1}{2} \lambda \tau\left(u^{\prime}, x, y\right)} \leq \zeta L^{-2}$. Thus it suffices to estimate the probability for the event that (4.120) happens for, say, some $x, y \in\left(L^{-(n+1)} \mathbb{Z}\right)^{d}$ with $\left|x-u^{\prime}\right|,\left|y-u^{\prime}\right|<L$. Let

$$
f(\cdot, \cdot)=\frac{1}{2} L^{2 n d} \delta_{x, \cdot} \delta_{y, \cdot} \exp \left(2 \lambda \tau\left(u^{\prime}, x, y\right)\right),
$$

with $\delta$ the Kronecker delta, so that $\|f\|_{u^{\prime}}=\frac{1}{2}$ and $\left(\sigma_{u^{\prime}}^{1}, 2 f\right)=\sigma_{u^{\prime}}^{1}(x, y) \exp \left(2 \lambda \tau\left(u^{\prime}, x, y\right)\right)$. Using (4.118) with $k=\zeta \epsilon_{n+1}^{1-3 \gamma}$, using $\exp \left(\frac{1}{2} \lambda \tau\left(u^{\prime}, x, y\right)\right) \geq 1$, and remembering that $\delta_{n+1}=L^{-(n+1) \gamma} \epsilon_{n+1}^{2-6 \gamma}$, we infer that the probability in question is bounded from above by

$$
2 L^{2(n+2) d} \exp \left(-L^{(n+1) \gamma}\right),
$$


where $L^{2(n+2) d}$ bounds the number of pairs $(x, y)$ with $\left|x-u^{\prime}\right|,\left|y-u^{\prime}\right|<L$. This implies the claim.

To prove Proposition 4.3, we need the following

Lemma 4.14. There exists a $C$, where $C \rightarrow \infty$ as $\epsilon \rightarrow 0$, such that if $K=C \log L$, and $n \geq m_{0}$, where

$$
L^{\frac{\gamma m_{0}}{2}}=n_{0}
$$

with $n_{0}$ defined in (4.21), then, for any $A \subset \mathbb{Z}^{d+1}$, any $\left\{N_{z}\right\}_{z \in A}$, with $N_{z} \neq 0$,

$$
\mathbb{P}\left(\left\{N_{n, z}=N_{z}\right\}_{z \in A}\right) \leq \exp \left(-K n|A|-2 K \sum_{z \in A} N_{z}\right)
$$

implies that (4.122) holds also for $N_{n+1}$, with $K$ replaced by $K^{\prime}=K\left(1-n^{-2}\right)$.

Proof. We set again $t^{\prime}=0$ (see Remark 4.8). Using the recursion relation (4.24), we can write, for any $\left(N_{z^{\prime}}^{\prime}\right)_{z^{\prime} \in A^{\prime}}, N_{z^{\prime}}^{\prime} \neq 0$,

$$
\begin{gathered}
\mathbb{P}\left(\left\{N_{n+1, z^{\prime}}=N_{z^{\prime}}^{\prime}\right\}_{z^{\prime} \in A^{\prime}}\right)= \\
(4.123) \sum_{B^{\prime} \subset A^{\prime}} \mathbb{P}\left(\left\{\tilde{N}_{z^{\prime}}=N_{z^{\prime}}^{\prime}+1-\left(n+n_{0}\right) \log L, r_{z^{\prime}} \neq 0\right\}_{z^{\prime} \in B^{\prime}} \&\left\{\tilde{N}_{z^{\prime}}=N_{z^{\prime}}^{\prime}+1\right\}_{z^{\prime} \in A^{\prime} \backslash B^{\prime}}\right)
\end{gathered}
$$

where we recall that, by (4.20) $) r_{z^{\prime}} \neq 0$ means that $r_{z^{\prime}}=\left(n_{0}+n\right) \log L$. Using Hölder's inequality, the summand is less than

$$
\begin{aligned}
& \mathbb{P}\left(\left\{\tilde{N}_{z^{\prime}}=N_{z^{\prime}}^{\prime}+1-\left(n_{0}+n\right) \log L\right\}_{z^{\prime} \in B^{\prime}} \&\left\{\tilde{N}_{z^{\prime}}=N_{z^{\prime}}^{\prime}+1\right\}_{z^{\prime} \in A^{\prime} \backslash B^{\prime}}\right)^{\left(1-\frac{1}{n^{2}}\right)} \\
& \cdot \mathbb{E}\left(\prod_{z^{\prime} \in B^{\prime}} \mathbb{1}\left(r_{z^{\prime}} \neq 0\right)\right)^{\frac{1}{n^{2}}} .
\end{aligned}
$$

Using the inductive assumption (4.122) the first factor is bounded by

$$
\prod_{z^{\prime} \in A^{\prime}}\left(\sum_{A_{z^{\prime}}} \sum_{\left\{N_{z}\right\}_{z \in A_{z^{\prime}}}} \exp \left[-K^{\prime} n\left|A_{z^{\prime}}\right|-2 K^{\prime} \sum_{z \in A_{z^{\prime}}} N_{z}\right]\right):=\prod_{z^{\prime} \in A^{\prime}} P_{z^{\prime}}
$$

where the sum $\sum_{A_{z^{\prime}}}$ runs, for $z^{\prime}=\left(u^{\prime}, 0\right)$, over all non empty subsets $A_{z^{\prime}} \subset L \mathbf{u}^{\prime} \times$ $\left[0, L^{2}-1\right]$, and the sum over $\left\{N_{z}\right\}$ runs over $N_{z} \neq 0$, satisfying the constraint

$$
\sum_{z \in A_{z^{\prime}}} N_{z}= \begin{cases}N_{z^{\prime}}^{\prime}+1-\left(n_{0}+n\right) \log L, & \text { if } z^{\prime} \in B^{\prime} \\ N_{z^{\prime}}^{\prime}+1, & \text { if } z^{\prime} \in A^{\prime} \backslash B^{\prime} .\end{cases}
$$

To estimate $P_{z^{\prime}}$ let us first observe that we have an a priori bound for all $x$ and $n$,

$$
N_{n, x} \leq L^{(3+d) n} n_{0} .
$$

Indeed, let $N_{n}=\sup _{z} N_{n, z}$. Then, we get from (4.2344.24) that

$$
N_{n+1} \leq L^{2+d} N_{n}+\left(n_{0}+n\right) \log L .
$$

From this, we get easily (4.127).

Let first $z^{\prime} \in A^{\prime} \backslash B^{\prime}$ and write $A_{z^{\prime}}=A$. By (4.126)

$$
P_{z^{\prime}}=\sum_{A} \mathcal{N}(|A|) \exp \left[-K^{\prime} n|A|-2 K^{\prime}\left(N_{z^{\prime}}^{\prime}+1\right)\right]
$$

where $\mathcal{N}(|A|)$ is the number of choices for $\left\{N_{z}\right\}$. For $|A|=1$ there is only one possible $z$ for which $N_{z}=N_{z^{\prime}}^{\prime}+1$ and thus $\mathcal{N}(1)=1$. For $|A|>1, \mathcal{N}(|A|) \leq\left(C L^{(3+d) n} n_{0}\right)^{|A|}$ 
since each $N_{z}$ takes a discrete set of values, by (4.23,4.24), and is bounded by (4.127). Since the sum over $A$ runs over subsets of a set of cardinality $L^{2+d}$, and thus contains less than $L^{(2+d)|A|}$ terms for given $|A|$, we infer

$$
P_{z^{\prime}} \leq \sum_{M=1}^{L^{2+d}} \mathcal{N}_{M} \exp \left[-K^{\prime} n M-2 K^{\prime}\left(N_{z^{\prime}}^{\prime}+1\right)\right]
$$

with $\mathcal{N}_{1}=L^{2+d}$ and $\mathcal{N}_{M}=\left(C L^{(3+d)(n+1)} n_{0}\right)^{M}$ for $M>1$.

Taking $K=C \log L$, for $C$ large, implies $K^{\prime}>\frac{1}{2} C \log L$ (since $n \geq m_{0}$, we may assume $n \geq 2)$. We then use $\exp \left[-K^{\prime} n(M-1)\right] \leq \exp \left[-K^{\prime}(n+1) M / 4\right]$, for $M>1$, the bounds $\exp \left[-K^{\prime}(n+1) M / 8\right] \leq \frac{1}{2} L^{-2-d}\left(C L^{(3+d)(n+1)}\right)^{-M}$ and $\exp \left[-K^{\prime} n M / 8\right] \leq n_{0}^{-M}$, which hold for $C$ large in $K^{\prime}>\frac{1}{2} C \log L$, and the fact that $n \geq m_{0}$, see (4.121), in order to bound the summand by $\frac{1}{2} L^{-2-d} \exp \left[-K^{\prime}(n+1)-2 K^{\prime} N_{z^{\prime}}^{\prime}\right]$, for $M>1$.

For $M=1, \mathcal{N}_{1}=L^{2+d}$ and we can use a factor $\exp \left[-K^{\prime}\right]$ to bound that term by $\frac{1}{2} L^{-2-d} \exp \left[-K^{\prime}(n+1)-2 K^{\prime} N_{z^{\prime}}^{\prime}\right]$.

Thus, since the sum over $M$ contains $L^{2+d}$ terms,

$$
P_{z^{\prime}} \leq \frac{1}{2} \exp \left[-K^{\prime}(n+1)-2 K^{\prime} N_{z^{\prime}}^{\prime}\right]
$$

for $z^{\prime} \in A^{\prime} \backslash B^{\prime}$.

Now consider $z^{\prime} \in B^{\prime}$. Proceeding exactly as above, but using the first equality in (4.126), we get that

$$
P_{z^{\prime}} \leq \frac{1}{2} \exp \left[-K^{\prime}(n+1)-2 K^{\prime} N_{z^{\prime}}^{\prime}+2 K^{\prime}\left(n_{0}+n\right) \log L\right]
$$

We will prove below that

$$
\mathbb{E}\left(\prod_{z^{\prime} \in B^{\prime}} \mathbb{1}\left(r_{z^{\prime}} \neq 0\right)\right) \leq \exp \left(-c^{\prime} L^{(n+1) \gamma}\left|B^{\prime}\right|\right)
$$

Now, insert (4.128) and (4.129) in (4.125); then, insert the result and (4.130) in (4.124) to obtain

$\left(\right.$ (4.124) $\leq 2^{-\left|A^{\prime}\right|} \exp \left[\left(-\frac{c^{\prime} L^{(n+1) \gamma}}{n^{2}}+2 K^{\prime}\left(n_{0}+n\right) \log L\right)\left|B^{\prime}\right|-K^{\prime}(n+1)\left|A^{\prime}\right|-2 K^{\prime} \sum_{z^{\prime} \in A^{\prime}} N_{z^{\prime}}^{\prime}\right]$.

Substitute this result into the sum (4.123). Since

$$
\exp \left[-\frac{c^{\prime} L^{(n+1) \gamma}}{n^{2}}+2 K^{\prime}\left(n_{0}+n\right) \log L\right] \leq 2^{-1}
$$

which follows from $n \geq m_{0}$ and $K^{\prime} \leq K=C \log L$, since we have both $L^{(n+1) \gamma / 2} \geq$ $C(\log L)^{2} n^{3}$, for $L$ large, and $L^{(n+1) \gamma / 2} \geq n_{0}$, see (4.121). Note that we can let $C \rightarrow \infty$, when $\epsilon \rightarrow 0$, since $n_{0}, m_{0} \rightarrow \infty$ as $\epsilon \rightarrow 0$, see (4.21), (4.121).

We may use

to conclude the iteration of (4.122).

$$
2^{-\left|A^{\prime}\right|} \sum_{B^{\prime} \subset A^{\prime}} 2^{-\left|B^{\prime}\right|} \leq 1
$$

To prove (4.130), decompose $\mathbb{Z}^{d+1}$ into $2^{d+1}$ sublattices $\mathbb{L}_{\ell}$ indexed by $\ell=1, \ldots, 2^{d+1}$, such that if $z_{1}=\left(u_{1}, t_{1}\right), z_{2}=\left(u_{2}, t_{2}\right)$ belong to the same sublattice, we have $\left|z_{1}-z_{2}\right| \geq$ 2. Use the Hölder inequality, as in the derivation of (4.115), to reduce the proof of 
(4.130) to the case where $B^{\prime}$ is included in one of those sublattices. Now apply (2.4): the support of the functions $F_{i}$ are, in the original lattice, at a distance at least $L^{n+1}$ and $\left|A_{i}\right| \leq L^{(n+1)(d+2)}$. Since $\exp \left(2\left|A_{i}\right| e^{-c L^{n+1}}\right) \leq C$ we conclude by using Corollary 4.13 to estimate (4.130) for $B^{\prime}$ reduced to a point (and absorbing thus the constant $C)$.

To finish the proof of Proposition 4.3, observe that we have $r_{z^{\prime}}=0 \forall z^{\prime}$, for $n<m_{0}$, which implies that $N_{n}=0, D_{n}=\emptyset$ and $\ell_{n}=0$ for $n<m_{0}$ (see (4.22,4.25) and (4.14.3) ). To see this, note that this holds for $n=0$ and that we get inductively, from (4.84) that the linear term in the iteration (4.30) of $b$ is bounded by:

$$
\left\|\mathcal{L} b_{n}\right\|_{\lambda} \leq C L\left\|b_{n}\right\|_{\lambda}
$$

and, as long as this remains smaller than $\epsilon_{n}^{1-3 \gamma}$, one can show, as in the proof of (4.71), that the nonlinear contributions are smaller, of order $\epsilon_{n}^{1-\gamma}$. This means that $\left\|b_{n}\right\|_{\lambda}$ and in particular $\left\|\sigma^{1}\right\|_{\lambda}$ is bounded by $(C L)^{n} \epsilon$. Thus, $r_{z^{\prime}}=0 \forall z^{\prime}$ as long as this is smaller than $\frac{1}{8} \epsilon_{n}^{1-3 \gamma}$ i.e. as long as $C^{n}\left(L^{n} \epsilon\right)^{3 \gamma} \leq \frac{1}{8}$, which is true for $\epsilon$ small and $n<m_{0}$, since then $L^{n} \leq n_{0}^{2 / \gamma}$, see (4.121), $C^{n}$ is also bounded by a small power of $n_{0}$, and (4.21) means that $n_{0} \leq|\log \epsilon|$.

Thus, Proposition 4.3 holds trivially for $n<m_{0}$. For $n \geq m_{0}$, where we can assume that $m_{0}$ is larger than 3, Lemma 4.14 implies inductively Proposition 4.3,

4.13. Proof of Proposition 4.5. We start with the study of the RG iteration for $T$ :

Lemma 4.15. There exist $r, c>0$ such that for all $n \geq 0, \hat{\mathcal{T}}_{n}$, defined in (3.13), (2.16), is analytic in $|\operatorname{Im} k|<r^{2} L^{\frac{n}{4}}$ and for such $k$

$$
\hat{\mathcal{T}}_{n}(k)=\left(1+\mathcal{O}\left(L^{-2 n}|k|^{4}\right)\right) e^{-\frac{D_{0}}{2 d} k^{2}}
$$

if $|k| \leq r L^{\frac{n}{4}}$ and

$$
\left|\hat{\mathcal{T}}_{n}(k)\right| \leq e^{-c L^{\frac{n}{2}}}
$$

otherwise.

Proof. Our assumption (iv) implies that $\hat{T}(k)$ is analytic in a neighborhood of $\mathbb{T}^{d}$. Combining with Assumption (vi), we conclude that, for $r$ small enough, $|\hat{T}(k)| \leq \rho(r)<$ 1 for $|\operatorname{Re} k|>r,|\operatorname{Im} k| \leq r^{2}$. This implies (4.134) for $|k|>r L^{n},|\operatorname{Im} k| \leq r^{2} L^{\frac{n}{4}}$.

Assumption (iii) in turn gives the representation (2.17) near the origin, which means that $\hat{T}(k)=e^{-\frac{D_{0}}{2 d} k^{2}}\left(1+\mathcal{O}\left(|k|^{4}\right)\right)$ for $|k| \leq r$. This implies (4.133) for $|k| \leq r L^{n}$, in particular for $|k| \leq r L^{\frac{n}{4}},|\operatorname{Im} k| \leq r^{2} L^{\frac{n}{4}}$. Since

$$
\left|e^{-\frac{D_{0}}{2 d} k^{2}}\right|=e^{-\frac{D_{0}}{2 d}\left((\operatorname{Re} k)^{2}-(\operatorname{Im} k)^{2}\right)} \leq e^{-\frac{D_{0}}{4 d}|k|^{2}}
$$

for $\frac{1}{2}|k|>|\operatorname{Im} k|$, the claim (4.134) holds also for $r L^{\frac{n}{4}}<|k| \leq r L^{n},|\operatorname{Im} k| \leq r^{2} L^{\frac{n}{4}}$, if $r$ is taken small enough.

The Lemma implies that $k \hat{\mathcal{T}}_{n}(k)$ has similar bounds in the strip, hence it is integrable there (recall that Re $k$ is on the $L^{n}$ torus) and thus it is exponentially decaying and we deduce (for $\lambda$ small)

$$
\left\|\mathcal{T}_{n}\right\|_{2 \lambda}+\left\|\nabla \mathcal{T}_{n}\right\|_{2 \lambda} \leq C
$$


Next, write again $T$ for $T_{n}$ and $T^{\prime}$ for $T_{n+1}$. Recall (4.32):

$$
T^{\prime}=L^{d} \mathcal{S}_{L} T^{L^{2}}+\mathbb{E} \nabla \cdot \mathcal{N}^{1} .
$$

We need

Lemma 4.16. There is a $\eta>0$ s.t. $\beta:=\mathbb{E} \nabla \cdot \mathcal{N}^{1}$ satisfies

$$
\|\beta\|_{2 \lambda}+\|\nabla \beta\|_{2 \lambda} \leq \epsilon_{n+1}^{\eta}
$$

Proof. Recall that $\mathcal{N}^{1}$ is a sum of products $\Pi$ (see (4.31)). We use two simple bounds for such products.

Note first that $\mathbb{E} \Pi$ is a function of $(x, y)$ only. So, $\|\mathbb{E} \Pi\|_{2 \lambda} \leq \sup _{v \in \mathbb{Z}^{d}} \mathbb{E}\left(\sum_{u}\|\Pi\|_{u, v} e^{2 \lambda|u-v|}\right)$, and we get, using (4.27):

$$
\|\mathbb{E} \Pi\|_{2 \lambda} \leq \sup _{v \in \mathbb{Z}^{d}} \mathbb{E}\left(\sum_{A, u} \sum_{\mathcal{D}}\left\|\Pi_{t, \mathcal{D}, A}\right\|_{u, v} e^{2 \lambda l(\mathcal{D}, A, t, u, v)} e^{-2 \lambda \tau(\mathcal{D} \cup u \cup v)}\right)
$$

Writing $1=e^{-N_{t}(\mathcal{D})} e^{+N_{t}(\mathcal{D})}$, and taking the sup over $u, \mathcal{D}$, we get:

$$
\begin{aligned}
& \sum_{A, u, \mathcal{D}}\left\|\Pi_{t, \mathcal{D}, A}\right\|_{u, v} e^{2 \lambda l(\mathcal{D}, A, t, u, v)} e^{-2 \lambda \tau(\mathcal{D} \cup u \cup v)} \\
& \leq\left(\sum_{A, u, \mathcal{D}}\left\|\Pi_{t, \mathcal{D}, A}\right\|_{u, v} e^{2 \lambda l(\mathcal{D}, A, t, u, v)} e^{-N_{t}(\mathcal{D})}\right)\left(\sup _{u \in \mathbb{Z}^{d}, \mathcal{D}} e^{-2 \lambda \tau(\mathcal{D} \cup u \cup v)} e^{N_{t}(\mathcal{D})}\right)
\end{aligned}
$$

Next, insert this in (4.138), take the supremum over the random variables $\omega$ for the sum $\sum_{A, u, \mathcal{D}}$, and replace the $\sup _{u \in \mathbb{Z}^{d}, \mathcal{D}}$ by a sum; we get:

$\|\mathbb{E} \Pi\|_{2 \lambda} \leq$

$$
0) \sup _{v \in \mathbb{Z}^{d}}\left(\left[\sup _{\omega} \sum_{A, u, \mathcal{D}}\left\|\Pi_{t, \mathcal{D}, A}\right\|_{u, v} e^{2 \lambda l(\mathcal{D}, A, t, u, v)} e^{-N_{t}(\mathcal{D})}\right]\left[\sum_{u \in \mathbb{Z}^{d}, \mathcal{D}} \mathbb{E}\left(e^{-2 \lambda \tau(\mathcal{D} \cup u \cup v)} e^{N_{t}(\mathcal{D})}\right)\right]\right)
$$

By Proposition $4.3 \mathbb{E}\left(e^{N_{t}(\mathcal{D})}\right)$ is bounded by $1+\exp (-c \lambda K)$, where $K$ is as in Proposition 4.3, and, for fixed $v$,

$$
\sum_{u \in \mathbb{Z}^{d}, \mathcal{D}} e^{-2 \lambda \tau(\mathcal{D} \cup u \cup v)} \leq C
$$

So, combining these last two bounds,

$$
\begin{aligned}
& \|\mathbb{E} \Pi\|_{2 \lambda} \leq C \sup _{\omega} \sup _{v \in \mathbb{Z}^{d}} \sum_{\mathcal{D}, A, u}\left\|\Pi_{t, \mathcal{D}, A}\right\|_{u, v} e^{2 \lambda l(\mathcal{D}, A, t, u, v)} e^{-N_{t}(\mathcal{D})} . \\
& =C \sup \|\Pi\|_{2 \lambda}
\end{aligned}
$$

and it suffices to bound $\|\Pi\|_{2 \lambda}$ uniformly in $\omega$.

The second bound uses independence. Consider first a product $\Pi$ such that there exists $i$ with $\alpha_{i}=1$ and no $j \in\{1, \ldots, N\}$ with $|i-j|=1$ and $\alpha_{j} \in\{1,2,3\}$ i.e. there is $b$ separated by two time units from other $B$ 's. This means that, in the original lattice, the distance between that $b$ and other $B$ 's is at least $L^{2 n}$; thus, we can use $\mathbb{E} b=0$ and the bound (2.3) which gives

$$
\|\mathbb{E} \Pi\|_{2 \lambda} \leq\|\Pi\|_{2 \lambda} e^{-c L^{2 n}},
$$


since the support of the functions in $\Pi$ is a power of $L^{n}$. The norm $\|\Pi\|_{2 \lambda}$ is bounded as in (4.71). Since the gradient is bounded by $C L^{n}$ (4.142) we may use $e^{-c L^{2 n}}$ to control the two gradients in $\nabla \nabla_{x} \cdot \Pi$, for the bound on $\nabla \beta$.

Finally, to control the two gradients in other cases, i.e. when there are no isolated $b$ 's, which we shall assume from now on, we use the translation invariance of the expectation values, which implies:

$$
\mathbb{E} \nabla_{x} \cdot \Pi(x, y)=-\mathbb{E} \nabla_{y} \cdot \Pi(x, y) .
$$

Let us bound now $\nabla \nabla_{x} \cdot \Pi$ using (4.141) and (4.143).

If $\alpha_{1}=4$ and $\alpha_{N}=4$ in (4.31), we can use (4.143) so that both gradients act on $T$ 's and, using inductively (4.57), we arrive at the bound (4.71) for $\|\nabla \nabla \cdot \Pi\|_{2 \lambda}$. Note that these $\alpha$ 's cannot take the value 5 , because there are no $\nabla T$ at the beginning or at the end of the products in (4.31), see (4.11).

Consider the other cases: if $\alpha_{1} \neq 4$ and $\alpha_{N}=4$, since there are no isolated $b$ 's, $\alpha_{1}=2$, i.e. the product starts with $b \nabla \cdot b$ (by (4.11), there is no $\nabla$ in the beginning). Then, we use (4.143) for one $\nabla$ and (4.50) to control $\nabla \cdot b \nabla \cdot b$. This is bounded by $\epsilon_{n+1}^{\eta}$ for $\eta$ small. If the product starts with $b \nabla \cdot b \nabla \cdot b$, we use (4.143) for one $\nabla$, (4.51) for the first $\nabla \cdot b$ and (4.50) for $\nabla \cdot b \nabla \cdot b$. This is again bounded by $\epsilon_{n+1}^{\eta}$ for $\eta$ small. Other terms are even smaller. We can proceed similarly if $\alpha_{1}=4$ and $\alpha_{N} \neq 4$, by using (4.143) for one of the $\nabla$ acting on $T_{\pi_{k}}$ ( see (4.11)), so that it acts on a $b$.

Finally, consider $\alpha_{1} \neq 4$ and $\alpha_{N} \neq 4$. If we have $\Pi=B^{2} T^{L^{2}-4} B^{2}$, we bound the norm of one $\nabla$ by $C L^{n}$ in $\nabla \nabla \cdot \Pi$, and use (4.50) for $\nabla \cdot B^{2}$ and (4.49) for $B^{2}$. The result is bounded by $\epsilon_{n+1}^{\eta}$ for $\eta$ small. For $\Pi=B^{2} \nabla \cdot b T^{L^{2}-5} B^{2}$, we get $\nabla \nabla \cdot \Pi=$ $\nabla \nabla \cdot B^{2} \nabla \cdot b T^{L^{2}-5} B^{2}$. Its norm is bounded by

$$
C(L) L^{n} \epsilon \epsilon_{n}^{3 / 2-4 \gamma},
$$

using (4.50) for $\nabla \cdot B^{2}$, (4.51) for $\nabla \cdot b$, (4.49) for $B^{2}$ and bounding the norm of the remaining $\nabla$ by $C L^{n}$. Since $\gamma<1 / 12$ this is bounded by $\epsilon_{n+1}^{\eta}$ for $\eta$ small.

Estimate (4.137) implies that the Fourier transform $\hat{\beta}(k)$ is analytic in $|\operatorname{Im} k|<2 \lambda$ and bounded there by $\epsilon_{n+1}^{\eta}$. By the symmetry assumption (iii) in Section 2.5 and the fact that $\hat{\beta}(0)=0$, which follows from (4.32) and $\hat{T}(0)=1=\hat{T}^{\prime}(0)$, the Taylor expansion reads

$$
\hat{\beta}(k)=\zeta k^{2}+\mathcal{O}\left(|k|^{4}\right)
$$

By a Cauchy's estimate

$$
|\zeta| \leq C \epsilon_{n+1}^{\eta}
$$

and

$$
\left|\hat{\beta}(k)-\zeta k^{2}\right| \leq C \epsilon_{n+1}^{\eta}|k|^{4},
$$

for $|k| \leq 1$, in the strip $|\operatorname{Im} k|<\frac{3 \lambda}{2}$. $\zeta$ will "renormalize" the effective diffusion constant. A convenient way to keep track of this "marginal" variable in the RG flow is the following inductive representation:

Lemma 4.17. $T_{n}$ can be decomposed as

$$
T_{n}(x)=\rho_{n}^{-d} \mathcal{T}_{n}\left(x / \rho_{n}\right)+t_{n}(x)
$$


where $\mathcal{T}_{n}$ is defined in (3.12), $\rho_{n}$ is a convergent sequence and there exists a constant A so that

$$
\left\|t_{n}\right\|_{\lambda}+\left\|\nabla t_{n}\right\|_{\lambda} \leq A \epsilon_{n}^{\eta}
$$

with $\hat{t}_{n}(k)=\mathcal{O}\left(|k|^{4}\right)$ at origin.

Proof. We proceed by induction, with primes referring to scale $n+1$. Set

$$
\rho^{2}=\rho^{2}-2 d \zeta D_{0}^{-1} .
$$

with $\rho_{0}=1$. The first term in (4.136) can be written as

$$
L^{d} \mathcal{S}_{L} T^{L^{2}}=L^{d} \mathcal{S}_{L}(\tilde{\mathcal{T}}+t)^{L^{2}}=\rho_{n}^{-d} \mathcal{T}^{\prime}\left(\cdot / \rho_{n}\right)+\tau
$$

where $\tilde{\mathcal{T}}_{n}(\cdot)=\rho_{n}^{-d} \mathcal{T}\left(\cdot / \rho_{n}\right)$, and

$$
\tau=L^{d} \sum_{m=1}^{L^{2}}\left(\begin{array}{l}
L^{2} \\
m
\end{array}\right) \mathcal{S}_{L} t^{m} \tilde{\mathcal{T}}^{L^{2}-m}
$$

Letting

$$
r:=\rho^{-d} \mathcal{T}^{\prime}(\cdot / \rho)-\rho^{\prime-d} \mathcal{T}^{\prime}\left(\cdot / \rho^{\prime}\right),
$$

whose Fourier transform is, using (4.133):

$$
\hat{r}(k):=\hat{\mathcal{T}}^{\prime}(\rho k)-\hat{\mathcal{T}}^{\prime}\left(\rho^{\prime} k\right)=-\frac{D_{0}}{2 d} k^{2}\left(\rho^{2}-\rho^{\prime 2}\right)+\mathcal{O}\left(|k|^{4}\right)=-\zeta k^{2}+\mathcal{O}\left(|k|^{4}\right),
$$

we get from (4.136) and (4.150) that $T^{\prime}=\rho^{-d} \mathcal{T}^{\prime}\left(\cdot / \rho^{\prime}\right)+t^{\prime}$, with

$$
t^{\prime}=r+\tau+\beta \text {. }
$$

Using (4.153), (4.144) for $r+\beta$, and using $\hat{t}(k)=\mathcal{O}\left(|k|^{4}\right)$ in (4.151), we get that $\hat{t}^{\prime}=\mathcal{O}\left(|k|^{4}\right)$.

By (4.145), (4.149),

$$
\left|\rho^{\prime}-\rho\right| \leq C \epsilon_{n+1}^{\eta},
$$

so that the sequence $\rho_{n}$ is convergent.

In order to iterate (4.148), consider first $r$. As in the derivation of (4.135) and using (4.153), 4.155), we get

$$
\|r\|_{\lambda}+\|\nabla r\|_{\lambda} \leq C \epsilon_{n+1}^{\eta} .
$$

As for $\tau$, separate the linear part in $t$ in (4.151):

$$
\tau=L^{d} L^{2} \mathcal{S}_{L} t \tilde{\mathcal{T}}^{L^{2}-1}+\tilde{\tau}:=\tau_{0}+\tilde{\tau},
$$

with

$$
\tilde{\tau}=L^{d} \sum_{m=2}^{L^{2}}\left(\begin{array}{c}
L_{m}^{2} \\
{ }^{2}
\end{array}\right) \mathcal{S}_{L} t^{m} \tilde{\mathcal{T}}^{L^{2}-m} .
$$

This last term is of the same form as $\Pi$ in (4.31) and is bounded using the inductive hypotheses (4.148) and (4.135),

$$
\|\tilde{\tau}\|_{\lambda}+\|\nabla \cdot \tilde{\tau}\|_{\lambda} \leq C(L) \epsilon_{n}^{2 \eta} \leq \epsilon_{n+1}^{\eta} .
$$


for $\epsilon$ small. We will now show that the term $\tau_{0}$ in (4.157), linear in $t$, contracts. This happens since $\hat{t}=\mathcal{O}\left(|k|^{4}\right)$. Indeed, in Fourier space, the first term $\tau_{0}$ in (4.157) equals

$$
\hat{\tau}_{0}(k)=L^{2} \hat{t}\left(\frac{k}{L}\right) \hat{\mathcal{T}}\left(\rho \frac{k}{L}\right)^{L^{2}-1}
$$

(4.148) implies that $\hat{t}$ is analytic in $|\operatorname{Im} k| l<\lambda$ and

$$
\sup _{|\operatorname{Im} k| l \leq \lambda}(1+|k|)|\hat{t}(k)| \leq C\left(\|t\|_{\lambda}+\|\nabla t\|_{\lambda}\right) .
$$

Hence, by a Cauchy estimate, and the fact that $\hat{t}=\mathcal{O}\left(|k|^{4}\right)$,

$$
\sup _{|k| \leq \frac{L \lambda}{2}}(1+|k|)\left|\hat{t}\left(\frac{k}{L}\right)\right| \leq C L^{-3}\left(\|t\|_{\lambda}+\|\nabla t\|_{\lambda}\right) .
$$

By Lemma 4.15,

$$
\sup _{|k| \geq \frac{L \lambda}{2}} \hat{\mathcal{T}}\left(\rho \frac{k}{L}\right)^{L^{2}-1} \leq e^{-c L^{2}}
$$

We conclude, using the obvious bound $\left|\hat{\mathcal{T}}\left(\rho \frac{k}{L}\right)\right| \leq 1$ for $|k| \leq \frac{L \lambda}{2}$, that:

$$
\sup _{|\operatorname{Im} k| l e q \frac{L \lambda}{2}}(1+|k|)\left|\hat{\tau}_{0}(k)\right| \leq C L^{-1}\left(\|t\|_{\lambda}+\|\nabla t\|_{\lambda}\right),
$$

which implies the desired contraction, using (4.148) inductively:

$$
\left\|\tau_{0}\right\|_{\lambda}+\left\|\nabla \tau_{0}\right\|_{\lambda} \leq C L^{-1}\left(\|t\|_{\lambda}+\|\nabla t\|_{\lambda}\right) \leq \frac{1}{2} A \epsilon_{n+1}^{\eta} .
$$

Using (4.156), (4.157), (4.158), (4.159), (4.137) to bound (4.154), and choosing $A$ so that

$$
C+2 \leq \frac{1}{2} A,
$$

for $C$ coming from (4.156), we get the iteration of (4.148).

To finish the proof of Proposition 4.5 we need to check (4.58). Write, using (4.147), $T_{n}^{t}=\left(\rho_{n}^{-d} \mathcal{T}_{n}\right)^{t}+\sum_{i=1}^{t} t_{n}^{i}\left(\rho_{n}^{-d} \mathcal{T}_{n}\right)^{t-i}$ It is readily verified, using (4.133), (4.134), that $\left(\rho_{n}^{-d} \mathcal{T}_{n}\right)^{t}$ satisfies (4.58). Now, use (4.135), (4.148), to bound $\left\|t_{n}^{i}\left(\rho_{n}^{-d} \mathcal{T}_{n}\right)^{t-i}\right\|_{\lambda} \leq A \epsilon_{n}^{\eta} C^{t}$, for any $i \geq 1$. Since $t \leq 2 L^{2}$, we may bound $(t-1) A \epsilon_{n}^{\eta} C^{t} \leq(t+1)^{-d / 2}$, which proves the bound (4.58) also for $\sum_{i=1}^{t} t_{n}^{i}\left(\rho_{n}^{-d} \mathcal{T}_{n}\right)^{t-i}$, since a bound on the $\|\cdot\|_{\lambda}$ norm implies the exponential decay in (4.58).

\section{THE NONLINEAR ANALYSIS.}

In this Section we will bound the nonlinear part of the map $f_{t}$. Recall that we wrote in Sect. 3 (2.18),

$$
D f_{t}(E)=T+\nabla b_{t}(E)
$$

and in Section 4 we have studied $b_{t}(0)$ and the composite $b_{t}(0) \nabla b_{t}(0)$. Now we need to extend that analysis to the $E$ dependence. Let $B_{t}^{1}(E)=b_{t}(E)$ and $B_{t}^{2}(E)=$ $b_{t+1}\left(f_{t}(E)\right) \nabla b_{t}(E)$, and $B^{3}=\nabla \cdot B^{2}, B^{4}=T, B^{5}=\nabla T$ as before. It turns out that for the nonlinear part we only need to keep track of the localization of $B_{t}$ in the region $D_{t}$ : we will establish inductively a decomposition

$$
B_{t}^{\alpha}(E)=\sum_{\mathcal{D} \subset D_{t}} B_{t, \mathcal{D}}^{\alpha}(E)
$$


Of course we have $B_{t, \mathcal{D}}^{\alpha}(0)=\sum_{A} B_{t, \mathcal{D} A}^{\alpha}$.

5.1. Inductive definition of $B$. We wish to show that the nonlinearity in $B^{\alpha}$ is irrelevant under the RG iteration. For $\alpha=2$ the reason for this will be the same as for the $E=0$ part. However, for $\alpha=1$ i.e. for $b$, we need to show the irrelevancy of its derivative $D b$ as explained in Sect. 3.6. To study its iteration consider the iterative formula (3.18) for the second derivative $D^{2} f$. We have outlined in Sect. 3.6. the argument that on the linear level $D b$ contracts. However, we face again the problem that in the nonlinear terms $\nabla b$ and $\nabla D b$ will enter and they are not small in our norm. Hence as for the iteration of $b$ we need to iterate bounds for composites involving $D b$. We denote $D b$ by $\mathcal{C}^{1}$. The composites that are analogous to $B^{2}$ are

$$
\left(\mathcal{C}_{t}^{2}, \mathcal{C}_{t}^{3}, \mathcal{C}_{t}^{4}\right):=\left(b_{t+1} \nabla \cdot D b_{t}, D b_{t+1}\left(\nabla \cdot b_{t} \otimes 1\right), D b_{t+1}\left(\nabla \cdot b_{t} \otimes \nabla \cdot b_{t}\right)\right),
$$

where we use the notation (3.19), and the ones analogous to $B^{3}$ are their divergences $\nabla \cdot \mathcal{C}^{\alpha}:=\mathcal{C}^{\alpha+3}$, for $\alpha=2,3,4$.

Let us introduce some notation: $I$ stands for a set $\left\{\left(\alpha_{i}, t_{i}\right)\right\}$ with $t_{i} \in\left[0,2 L^{2}-1\right]$ and $\alpha_{i} \in\{1, \ldots, 5\}$. Denote

$$
B_{I}:=B_{t_{1}}^{\alpha_{1}}\left(E_{t_{1}}\right) \ldots B_{t_{N}}^{\alpha_{N}}\left(E_{t_{N}}\right)
$$

with, for $t_{i} \geq 1$,

$$
E_{t_{i}}=f_{t_{i}-1} \circ \cdots \circ f_{0}(E) \text { with } \quad E=L^{-d} E^{\prime}(\cdot / L) .
$$

With this notation

$$
D f_{t^{\prime}}^{\prime}\left(E^{\prime}\right)=L^{d} \mathcal{S}_{L} T^{L^{d}}+\nabla \cdot \mathcal{M}^{1}\left(E^{\prime}\right)
$$

where $\mathcal{M}^{1}$ is a sum

$$
\mathcal{M}^{1}=L^{d-1} \sum_{I} \mathcal{S}_{L} B_{I}
$$

Thus, see (5.1), (3.8),

$$
b^{\prime}\left(E^{\prime}\right)=\mathcal{M}^{1}\left(E^{\prime}\right)-\mathbb{E} \mathcal{M}^{1}(0) .
$$

Similarly we have

$$
B_{t^{\prime}}^{\prime}=(\mathcal{L} B)_{t^{\prime}}+\mathcal{M}^{2}(B)_{t^{\prime}}
$$

where we write explicitly the linear term, with $\mathcal{L}$ given by the analogue of (4.14),

$$
(\mathcal{L} B)_{t^{\prime}}\left(E^{\prime}\right):=L^{d-1} \mathcal{S}_{L} T^{L^{2}-1} B_{L^{2}\left(t^{\prime}+1\right)-1}\left(E_{L^{2}\left(t^{\prime}+1\right)-1}\right) T^{L^{2}-1} .
$$

Let us next derive the recursion relation for the $\mathcal{C}^{\alpha}$. Consider first $\mathcal{C}^{1}$ i.e. $D b$. Inserting to (3.18) the decomposition (5.1) and expand as in (4.9) and (4.11). This way $\mathcal{C}^{1}$ becomes a sum of terms of the form

$$
L^{d-1} \mathcal{S}_{L} Q_{\pi} \mathcal{C}_{t}\left(\nabla Q_{\pi^{\prime}} \otimes \nabla Q_{\pi^{\prime \prime}}\right)
$$

where

$$
Q_{\pi}=T_{\pi_{k}} b_{\tau_{k-1}}(\nabla \cdot b)_{\pi_{k-1}^{\prime}} \nabla \cdot T_{\pi_{k-2}} \ldots b_{\tau_{2}}(\nabla \cdot b)_{\pi_{2}^{\prime}} T_{\pi_{1}},
$$

as in (4.11). We may express this again in terms of the products (5.4) and the composites invoving $D b$ (5.3) and their derivatives:

$$
\mathcal{C}^{\prime 1}=\tilde{\mathcal{L}}_{1} \mathcal{C}^{1}+L^{d-1} \sum_{\left\{I_{i}\right\}} \mathcal{S}_{L} B_{I_{1}} \mathcal{C}_{I_{2}}\left(B_{I_{3}} \otimes B_{I_{4}}\right) .
$$


where $\tilde{\mathcal{L}}_{1}=\tilde{\mathcal{L}}$ defined in $(3.20)$ and where $I_{2}=\{(\alpha, t)\}, \alpha \in\{1, \ldots, 7\}$. It is easy to see that all seven of them can occur!

As in the case of $B^{2}$ we obtain for $\mathcal{C}^{\alpha}$ with $\alpha>1$ an expansion as in eq. (5.11) on the time interval $\left[0,2 L^{2}-1\right]$. For $\alpha=2,5$ the times in $I_{1}$ lie on $\left[L^{2}, 2 L^{2}-1\right]$ and those on $I_{k} k>1$ lie on $\left[0, L^{2}-1\right]$ and, for $\alpha=3,4,6,7$, we have $I_{1}, I_{2} \subset\left[L^{2}, 2 L^{2}-1\right]$, $I_{3}, I_{4} \subset\left[0, L^{2}-1\right]$.

We have, since $\nabla$ acts on a $T$, as in (4.74), that:

$$
\tilde{\mathcal{L}}_{\alpha}=0, \quad \alpha>4 .
$$

We also need to localize

$$
\mathcal{C}_{t}^{1}(E)=\sum_{\mathcal{D} \subset D_{t}} \mathcal{C}_{t, \mathcal{D}}^{1}(E)
$$

and similarly for the $\alpha>1$ with $\mathcal{D}=\left(\mathcal{D}_{t}, \mathcal{D}_{t+1}\right)$ as in (4.15). We will now explain how (5.2) and (5.13) are carried through the induction.

First write, as in (4.34) and (4.35)

$$
B_{t_{1}}^{\alpha}=\sum_{\mathcal{D}_{i}} B_{t_{i}, \mathcal{D}_{i}}^{\alpha}=\sum_{\tilde{\mathcal{D}}_{i}} \tilde{B}_{t_{i}, \tilde{\mathcal{D}}_{i}}^{\alpha}
$$

This way we end up with

$$
\mathcal{M}^{1}\left(E^{\prime}\right)=L^{d-1} \sum_{J} \mathcal{S}_{L} \tilde{B}_{J}
$$

with $\tilde{B}_{J}=\tilde{B}_{t_{1}, \tilde{\mathcal{D}}_{1}}^{\alpha_{1}}\left(E_{t_{1}}\right) \ldots \tilde{B}_{t_{N}, \tilde{\mathcal{D}}_{N}}^{\alpha_{N}}\left(E_{t_{N}}\right)$ and $J$ stands for the set $\left\{\left(\alpha_{i}, t_{i}, \tilde{\mathcal{D}}_{i}\right)\right\}$.

Localize (5.15) by collecting all terms with $\left[\cup \tilde{\mathcal{D}}_{i}\right]=\mathcal{D}^{\prime}$

$$
\mathcal{M}^{1}\left(E^{\prime}\right)=\sum_{\mathcal{D}^{\prime}} \mathcal{M}_{\mathcal{D}^{\prime}}^{1}\left(E^{\prime}\right)
$$

which we write below as $\mathcal{M}_{\emptyset}^{1}\left(E^{\prime}\right)+\sum_{\mathcal{D}^{\prime} \neq \emptyset} \mathcal{M}_{\mathcal{D}^{\prime}}^{1}\left(E^{\prime}\right)$. In the same way we localize

$$
\mathcal{C}^{\prime \alpha}=L^{d-1} \sum \mathcal{S}_{L} \tilde{B}_{J_{1}} \tilde{\mathcal{C}}_{J_{2}}\left(\tilde{B}_{J_{3}} \otimes \tilde{B}_{J_{4}}\right)=\sum_{\mathcal{D}^{\prime}} \mathcal{C}_{\mathcal{D}^{\prime}}^{\prime \alpha}
$$

where again $J_{2}$ is the singleton $\left\{\left(t_{2}, \alpha_{2}, \tilde{\mathcal{D}}_{2}\right)\right\}$ and we included the linear term in (5.11) to the sum as well.

Localization of $b^{\prime}$ is less obvious. Basically the idea is that $b^{\prime}\left(E^{\prime}\right)-b^{\prime}(0)$ contracts (unlike $b^{\prime}(0)$ !) because it is controlled by $D b^{\prime}$ which contracts. However, from (5.11) we see that $D b^{\prime}$ has terms that are quadratic in $B_{t}^{\alpha}$ for $t \in J_{3}, J_{4}$. Hence the bound for $D b^{\prime}$ will involve $e^{2 N_{t^{\prime} \mathcal{D}^{\prime}}}$ instead of the $e^{N_{t^{\prime} \mathcal{D}^{\prime}}}$ factor in the bound of $b^{\prime}$ (see eq. (5.29) below). Thus we need to be very careful not to propagate such factors from $D b^{\prime}$ to $b^{\prime}$.

Let $\mathcal{J}_{0}$ be the set of $J$ with all $\tilde{\mathcal{D}}_{i}=\emptyset$. Then

$$
D \mathcal{M}_{\emptyset}^{1}=L^{d-1} \sum_{J_{1}, J_{2}, J_{3} \in \mathcal{J}_{0}} \mathcal{S}_{L} \tilde{B}_{J_{1}} \tilde{\mathcal{C}}_{J_{2}}\left(\tilde{B}_{J_{3}} \otimes \tilde{B}_{J_{4}}\right)=\sum_{\mathcal{D}^{\prime}} \mathcal{N}_{\mathcal{D}^{\prime}}
$$

We set $b_{t^{\prime}, \emptyset}^{\prime}\left(E^{\prime}\right)=\mathcal{N}_{\emptyset}\left(E^{\prime}\right)$, which we write as:

$$
b_{t^{\prime}, \emptyset}^{\prime}\left(E^{\prime}\right)-b_{t^{\prime}, \emptyset}^{\prime}(0)=\int_{0}^{1} d \lambda \mathcal{N}_{\emptyset}\left(\lambda E^{\prime}\right) E^{\prime}
$$


and for $\mathcal{D}^{\prime} \neq \emptyset$,

$$
b_{t^{\prime}, \mathcal{D}^{\prime}}^{\prime}\left(E^{\prime}\right)-b_{t^{\prime} \mathcal{D}^{\prime}}^{\prime}(0)=\int_{0}^{1} d \lambda \mathcal{N}_{\mathcal{D}^{\prime}}\left(\lambda E^{\prime}\right) E^{\prime}+\mathcal{M}_{\mathcal{D}^{\prime}}^{1}\left(E^{\prime}\right)-\mathcal{M}_{\mathcal{D}^{\prime}}^{1}(0) .
$$

Denote the $\mathcal{D}=\emptyset$ and $\mathcal{D} \neq \emptyset$ parts of $b(E)$ by

$$
m_{t}(E)=b_{t, \emptyset}(E)-b_{t, \emptyset}(0)
$$

and

$$
M_{t}(E)=\sum_{\mathcal{D} \neq \emptyset} b_{t, \mathcal{D}}(E)-b_{t, \mathcal{D}}(0)
$$

so that we have:

$$
b_{t}(E)-b_{t}(0)=m_{t}(E)+M_{t}(E) \text {, }
$$

and

$$
b_{t}(E)=s_{t}+\ell_{t}+m_{t}(E)+M_{t}(E) .
$$

Note that these definitions avoid the problem with the $N$ factors since the only contribution to $b^{\prime}$ from $D b^{\prime}$ is from (5.18) where only the $J_{4}$ term can contribute a $e^{N_{t, \mathcal{D}}}$ factor. $\mathcal{N}$ will contract for reasons stated above and $\mathcal{M}_{\mathcal{D}^{\prime}}^{1}\left(E^{\prime}\right)$ for $\mathcal{D}^{\prime} \neq \emptyset$ will contract for the same reasons that $\ell=\mathcal{M}^{1}(0)-\mathbb{E}\left(\mathcal{M}^{1}(0)\right)$ contracted.

5.2. Inductive bounds. The analysis is now similar to the $E=0$ case, if only it is simpler due to less localization. We use the norm, analogous to (4.29), but without the $A$ sum:

$$
\left\|B_{t}^{\alpha}\right\|_{\lambda}=\sup _{E \in B_{\delta}^{+}} \sup _{v \in \mathbb{Z}^{d}} \sum_{\mathcal{D}, u}\left\|B_{t, \mathcal{D}}^{\alpha}\right\|_{u, v} e^{\lambda \tau(\mathcal{D}, u, v)} e^{-N_{t, \mathcal{D}}}
$$

where, for $\alpha=2, \mathcal{D}$ is a pair $\left(\mathcal{D}_{1}, \mathcal{D}_{2}\right)$, with the same conventions as in (4.29).

Note that since $f_{t}$ preserves $\int E d x$ the arguments (5.5) of the functions $B$ in (4.31) are in $B_{\delta}^{+}$if $E$ is. For convenience we also choose $\delta=\epsilon$.

Then we have:

Proposition 5.1. Let $\gamma$ be as in Proposition 4.1. Then, for all $t, n$,

$$
\begin{aligned}
&\|m\|_{\lambda} \leq \epsilon_{n}^{(1-2 \gamma)} \\
&\|M\|_{\lambda} \leq \epsilon_{n}^{(1-2 \gamma)} . \\
&\|B\|_{\lambda} \leq \epsilon_{n}^{(1-\gamma / 2)} \\
&\left\|B^{3}\right\|_{\lambda}=\|\nabla \cdot B\|_{\lambda} \leq \epsilon^{1-2 \gamma} L^{-n / 2} .
\end{aligned}
$$

As indicated above for the $\mathcal{C}^{\alpha}$ we have to use a slightly different definition of the norm:

$$
\left\|\mathcal{C}_{t}^{\alpha}\right\|_{\lambda}=\sup _{E \in B_{1}^{+}} \sup _{v, w \in \mathbb{Z}^{d}} \sum_{\mathcal{D}, u}\left\|\mathcal{C}_{t, \mathcal{D}}^{\alpha}\right\|_{u, v, w} e^{\lambda \tau(\mathcal{D}, u, v, w)} e^{-N_{t, \mathcal{D}}^{\alpha}}
$$

with $\|C\|_{u, v, w}=\sup _{x \in \mathbf{u}, y \in \mathbf{v}, z \in \mathbf{w}}|C(x, y, z)|$, and

$$
\begin{aligned}
& N_{t, \mathcal{D}}^{1}=2 N_{t, \mathcal{D}_{t}}, \\
& N_{t, \mathcal{D}}^{2}=2 N_{t, \mathcal{D}_{t}}+N_{t+1, \mathcal{D}_{t+1}}, \\
& N_{t, \mathcal{D}}^{\alpha}=N_{t, \mathcal{D}_{t}}+2 N_{t+1, \mathcal{D}_{t+1}}, \alpha \in\{3,4\} .
\end{aligned}
$$


Note that we introduce a $2 N_{t}$ instead of $N_{t}$ for the times $t$ where a $D b$ occurs. The bounds for the $\mathcal{C}$ are:

Proposition 5.2. For all $t, n$,

$$
\begin{aligned}
\|D m\|_{\lambda} & \leq C L^{-(1-\gamma) n} \\
\|D M\|_{\lambda} & \leq C L^{-(1-\gamma) n} .
\end{aligned}
$$

Moreover, for $\beta=2,3,4$

$$
\begin{aligned}
\left\|\mathcal{C}^{\beta}\right\|_{\lambda} & \leq \epsilon^{(1-2 \gamma)} L^{-(1-\gamma) n}, \\
\left\|\mathcal{C}^{3+\beta}\right\|_{\lambda}=\left\|\nabla \cdot \mathcal{C}^{\beta}\right\|_{\lambda} & \leq \epsilon_{n}^{1 / 2} .
\end{aligned}
$$

Remark 5.3. From (5.24,5.25) and (5.30,5.32) we get, as in (4.51)),

$$
\|\nabla \cdot b\|_{\lambda} \leq C \epsilon \epsilon_{n}^{-2 \gamma} \text {. }
$$

and

$$
\|\nabla \cdot D b\|_{\lambda} \leq C L^{\gamma n}
$$

Note also that we cannot deduce (5.24,5.25) from (5.30,5.31) by integration, because of the difference between the norms (5.23) and (5.28).

5.3. Proof of the Propositions. For $n=0$, the bounds in Proposition 5.1 follow by writing, see (5.1),

$$
m(E)=D f(E)-D F(0)=\int_{0}^{1} d \mu D^{2} F(\mu E) E
$$

using assumption (iv), $\delta=\epsilon$ and the fact that, for $n=0$, the norm of $\nabla$ is of order one. We have, for $n=0, D=\emptyset, M=0$. The bounds in Proposition 5.2, follow similarly, from assumption (iv) for $D m$, and by combining bounds on $m$ and $D m$ for $\mathcal{C}^{\beta}$.

The iteration of the bounds in the propositions goes as in Section 4.10. except that it is simpler due to the lack of the $A$ sums. Hence, we will be brief.

The bound (5.26) follows as in Section 4.10, see (4.91). The supremum over $E$ in the norms comes for free since for all $t$ we have $E_{t} \in B_{\delta}^{+}$. Thus, e.g. the operator (5.10) has the same bound (4.91) as in the $E=0$ case. The proof of (5.27) follows the one of (4.50), see (4.73), (4.74). Since the bounds here are different from the ones in Proposition 4.1, we have, instead of (4.73), using (5.34), (5.24), (5.25),

$$
\left\|\nabla \cdot \mathcal{N}^{2}\right\|_{2 \lambda} \leq C(L) \epsilon \epsilon_{n}^{1-4 \gamma}=C(L) \epsilon^{2-4 \gamma} L^{-(1-4 \gamma) n} \leq \frac{1}{2} \epsilon^{1-2 \gamma} L^{-(n+1) / 2},
$$

since $\gamma<\frac{1}{12}$ and $\epsilon$ is small. The bound on $\nabla \cdot \mathcal{L} B^{2}$ is similar to (4.74).

Let us consider first the nonlinear terms in the iteration of (5.24) 5.25$)$ and (5.30) 5.32 ).

Note that, with the norms (5.23), (5.28), and with (3.19), we have:

$$
\begin{aligned}
& \sup _{v, w} \sum_{\mathcal{D}, u}\left\|\mathcal{C}^{\alpha}\left(B_{1} \otimes B_{2}\right)_{\mathcal{D}}\right\|_{u, v, w} e^{\lambda \tau(\mathcal{D}, u, v, w)} \leq \\
& C \sup _{z_{1}, z_{2}} \sum_{\mathcal{D}, u}\left\|\mathcal{C}_{\mathcal{D}}^{\alpha}\right\|_{u, z_{1}, z_{2}} e^{\lambda \tau\left(\mathcal{D}, u, z_{1}, z_{2}\right)} . \\
& \sup _{v} \sum_{\mathcal{D}_{1}, z_{1}}\left\|B_{1 \mathcal{D}_{1}}\right\|_{z_{1}, v} e^{\lambda \tau\left(\mathcal{D}_{1}, z_{1}, v\right)} \sup _{w} \sum_{\mathcal{D}_{2}, z_{2}}\left\|B_{2 \mathcal{D}_{2}}\right\|_{z_{2}, w} e^{\lambda \tau\left(\mathcal{D}_{1}, z_{2}, w\right)} .
\end{aligned}
$$


With this, one can prove the analogue of Lemma 4.9; first, the analogue of (4.62), to bound products of the form $B_{I_{1}} \mathcal{C}_{I_{2}}\left(B_{I_{3}} \otimes B_{I_{4}}\right)$ in (5.11), with the norm (5.28) on the left hand side and a factor given by the norm (5.28) of $\mathcal{C}^{\alpha}$ on the right hand side. The $\exp (N)$ factors in the norms are dealt with eq. (4.66), which holds for the new definition (5.29) as well; since we have defined the new $N_{t^{\prime}, \mathcal{D}}^{\prime \alpha}$ so that we have $2 N_{t^{\prime}}$ whenever there is a derivative, we can control both the $2 N_{t}$ associated with $\mathcal{C}_{I_{2}}$ and the two $N_{t}$ 's coming from $B_{I_{3}} \otimes B_{I_{4}}$. Secondly, the analogue of the bound (4.63) can be proven for the norm (5.28) of $\tilde{\mathcal{C}}$ and the one of $\mathcal{C}$, as in the proof of Lemma 4.9, since the bound (4.69) holds also for (5.29).

The nonlinear terms in the iteration of $D m$ or $D M$, are given by (5.17), see (5.8), (5.11), (5.18 5.22). The leading terms are when all $B_{I_{k}}, k=1,3,4$, are products of $T$ 's and $\nabla T$ 's. Then, we have, in $J_{2} \alpha_{2} \neq 1$ (otherwise, it would be the linear term) and $\alpha_{2} \leq 4$ (otherwise the $\nabla$ would be integrated by parts to a $T$ ) and we can use inductively (5.32) and $C \epsilon^{(1-2 \gamma)} L^{-(1-\gamma) n} \leq \frac{1}{2} L^{-(1-\gamma)(n+1)}$, for $\epsilon$ small. All the other terms give rise to smaller contributions.

The same holds for the nonlinear terms in the iteration of $m$ or $M$, that are also of the form of (5.17), see (5.18 5.22), except for the nonlinear terms in $\mathcal{M}^{1}$, which are bounded in the same way as the nonlinear terms for $E=0$, see (4.71). We use (5.32) and $C \epsilon^{(1-2 \gamma)} L^{-(1-\gamma) n} \leq \frac{1}{2} \epsilon_{n}^{1-2 \gamma}$ to bound the contributions of $\mathcal{C}^{\beta}, \beta>1$ to $m$ and $M$ (we have $C$ here, not $C(L)$, since there is no sum over times in those contributions). The crucial observation, for the nonlinear terms in $\mathcal{N}$, is that the $\exp (N)$ factors work out: we have only one $N_{t}$ in the exponentials in the bound for $M^{\prime}$ (see (5.23)) but also in the bound for $\mathcal{N}$, since in (5.18) only $J_{4}$ can have $\tilde{\mathcal{D}}_{i} \neq \emptyset$, see (5.19).

Consider next the nonlinear contributions to the iteration of (5.32) and start with $\beta=2$. Since one cannot have a $\nabla$ in the leftmost matrix in the recursion (5.11), the leading nonlinear terms are proportional to

$$
T^{L^{2}-2} b_{L^{2}+1} \nabla \cdot \mathcal{C}_{L^{2}-1}^{\alpha}\left(T^{L^{2}-1} \otimes T^{L^{2}-1}\right)
$$

which, using recursively (5.24, 5.25), 5.33), is $\mathcal{O}\left(\epsilon_{n}^{\frac{3}{2}-2 \gamma}\right)$, and, for $\gamma<1 / 12$, this is smaller than $\frac{1}{2} \epsilon^{(1-2 \gamma)} L^{-(1-\gamma) n}$. We also have terms with $b$ and $D b$ separated by powers of $T$ or $\nabla T$ (the $\nabla$ in $\nabla D b$ being integrated by parts). These terms are bounded by combining (5.24, 5.25) and (5.30, 5.31) and are much smaller than $\epsilon^{(1-2 \gamma)} L^{-(1-\gamma) n}$.

Similarly, for $\beta=3,4$, the leading nonlinear terms are $T^{L^{2}-3} \mathcal{C}_{L^{2}+1}^{1}\left(B_{L^{2}-1}^{3} T^{L^{2}-1} \otimes 1\right)$ and $T^{L^{2}-3} \mathcal{C}_{L^{2}+1}^{1}\left(B_{L^{2}-1}^{3} T^{L^{2}-1} \otimes B_{L^{2}-1}^{3} T^{L^{2}-1}\right)$. The first one is, since $\mathcal{C}^{1}=D b$, using recursively (5.30, 5.31, 5.27), $\mathcal{O}\left(\epsilon^{1-2 \gamma} L^{-\left(\frac{3}{2}-\gamma\right) n}\right)$ which, for $\gamma<1 / 12$, is again smaller than $\frac{1}{2} \epsilon^{(1-2 \gamma)} L^{-(1-\gamma) n}$. The second term is smaller.

Next we turn to the linear RG for $\mathcal{C}^{\beta}$. For $\beta=1$, i.e. for $D m$ and $D M$, we get, as in (3.20):

$$
\begin{aligned}
& \left(\tilde{\mathcal{L}}_{1} \mathcal{C}^{1}\right)_{t^{\prime}}\left(x^{\prime}, y^{\prime}, z^{\prime}, E^{\prime}\right)= \\
& L^{d-1} \sum_{t \in I_{t^{\prime}}} \int d x d y d z T^{L^{2}-i-1}\left(L x^{\prime}-x\right) \mathcal{C}_{t}^{1}\left(x, y, z, E_{t}\right) T^{i}\left(y-L y^{\prime}\right) T^{i}\left(z-L z^{\prime}\right)
\end{aligned}
$$


We can now proceed as in (4.75) - (4.83) with the difference that in (4.77) we have also a $w$ sum; in (4.81), we have $\frac{\lambda}{12}(|u-v|+|u-w|)$ instead of $\frac{\lambda}{6}|u-v|$, and, here

$S_{i}\left(u, v, w, u^{\prime}, v^{\prime}, w^{\prime}\right):=\sup _{x^{\prime} \in \mathbf{u}^{\prime}, y^{\prime} \in \mathbf{v}^{\prime}, z^{\prime} \in \mathbf{w}^{\prime}} \int_{\mathbf{u}} d x \tilde{T}^{L^{2}-i-1}\left(L x^{\prime}-x\right) \int_{\mathbf{v}} d y \tilde{T}^{i}\left(y-L y^{\prime}\right) \int_{\mathbf{w}} d z \tilde{T}^{i}\left(z-L z^{\prime}\right)$,

with $\tilde{T}$ and $k$ as in (4.78), (4.80). Eq. (4.83) is then replaced by

$$
\sum_{u, v, w} e^{-\frac{\lambda}{12}(|u-v|+|u-w|)} S_{i}\left(u, v, w \cdot u^{\prime}, v^{\prime}, w^{\prime}\right) \leq C \min \left(i^{-d}, i^{-\frac{d}{2}}\left(L^{2}-i-1\right)^{-\frac{d}{2}}\right) \leq C L^{-d} i^{-\frac{d}{2}},
$$

since we can use twice the $L^{\infty}$ bound in (4.60) and use one $L^{1}$ bound and the factor $e^{-\frac{\lambda}{12}(|u-v|+|u-w|)}$ to control the sums. Summing $i^{-\frac{d}{2}}$ over $i$ leads to the bound, for $d \geq 2$,

$$
\left\|\tilde{\mathcal{L}}_{1} \mathcal{C}\right\|_{\lambda} \leq C L^{-1} \log L\|\mathcal{C}\|_{\lambda}
$$

The other $\tilde{\mathcal{L}}_{\alpha}, \alpha=2,3,4$, have similar bounds, in fact better ones since there is no sum over times. Combined with the bounds on the nonlinear terms the iteration of (5.30), (5.31) and (5.32) follows.

Consider then (5.33). We have again a term $\nabla \tilde{\mathcal{L}}_{\beta} \cdot \mathcal{C}^{\beta}$, which can be bounded, using (5.32), as in (4.74). Among the nonlinear contributions, for $\beta=2$, the largest one is

$$
\nabla \cdot b_{2 L^{2}-1} \nabla T^{L^{2}-2} \mathcal{C}_{L^{2}-1}^{1}\left(T^{L^{2}-1} \otimes T^{L^{2}-1}\right)
$$

i.e. $\mathcal{O}\left(\epsilon^{1-2 \gamma} L^{-(1-3 \gamma) n}\right)$ using (5.34) and (5.30, 5.31); this is smaller than $\frac{1}{2} \epsilon_{n+1}^{1 / 2}$ for $\gamma<1 / 12$, which fits to (5.33). For $\beta=3$ the leading nonlinear term is proportional to

$$
\nabla \mathcal{C}_{2 L^{2}-1}^{1}\left(\nabla T^{L^{2}-1} \otimes T^{L^{2}-1}\right)\left(b_{L^{2}-1} T^{L^{2}-1} \otimes 1\right)
$$

i.e. again, using (5.35) and (5.30, 5.31), $\mathcal{O}\left(\epsilon^{1-2 \gamma} L^{-(1-3 \gamma) n}\right)$, which is less than $\frac{1}{2} \epsilon_{n+1}^{1 / 2}$. For $\beta=4$ the nonlinear terms are smaller.

To finish the proofs,we need to bound the linear contributions to (5.24) and (5.25). Thus consider eqs. (5.19) and (5.20). The linear term comes from (5.18) with all the $\tilde{B}$ being powers of $T$ and $\mathcal{C}^{\alpha_{2}}=\mathcal{C}^{1}$; we use the bound (5.40) together with the estimate

$$
\left\|\mathcal{N}\left(\lambda E^{\prime}\right) E^{\prime}\right\|_{\lambda} \leq\left\|\mathcal{N}\left(\lambda E^{\prime}\right)\right\|_{\lambda}\left\|E^{\prime}\right\|_{1}
$$

which is obtained by writing

$$
\left(\mathcal{N}\left(\lambda E^{\prime}\right) E^{\prime}\right)(x, y)=\int \mathcal{N}\left(\lambda E^{\prime}\right)(x, y, z) E^{\prime}(z) d z
$$

$\int d z=\sum_{w \in \mathbb{Z}^{d}} \int_{\mathbf{w}} d z$ and $\int_{\mathbf{w}} E^{\prime}(z) d z \leq\left\|E^{\prime}\right\|_{1}$. We then use in (5.41) $\left\|E^{\prime}\right\|_{1}=\|E\|_{1} \leq$ $\delta=\epsilon$, where the first equality follows inductively from assumption (ii), and the bounds (5.30) on $\mathcal{C}^{1}$ for $\mathcal{N}$. This controls the linear contribution of the $\mathcal{N}$ terms in (5.19) and (5.20) and, with the previous bounds on the nonlinear terms in $\mathcal{N}$ and the $\mathcal{M}^{1}$ terms in (5.20), proves (5.24) and (5.25) 


\section{Proof of the main Results.}

We will prove (2.21) for

$$
D=\lim _{n \rightarrow \infty} \rho_{n} D_{0}
$$

where $\rho_{n}$ is the sequence in Lemma 4.17

Proof of Theorem 2.1 Using (3.2) and (3.3) with $t=0$, we have, $\forall x \in\left(L^{-n} \mathbb{Z}\right)^{d}$,

$$
L^{n d} E\left(L^{2 n}, L^{n} x\right)=E_{n}(1, x)=\int_{0}^{1} d \lambda \int d y D f_{n}\left(\lambda E_{n}\right)(x, y) E_{n}(y),
$$

with $E_{n}(y)=: E_{n}(0, y)=L^{n d} E\left(0, L^{n} y\right)$ and $D f_{n}=: D f_{n, 0}$, where, see (6.12),

$$
D f_{n}\left(\lambda E_{n}\right)(x, y)=T_{n}(x-y)+\nabla \cdot b_{n}\left(\lambda E_{n}\right)(x, y),
$$

with $b_{n}$ at $t=0$. Let

$$
\bar{\Omega}=\left\{\omega \mid \exists m, \text { such that } \forall \mathcal{D} \subset \mathbb{Z}^{d}, \forall n \geq m, N_{n}(\mathcal{D}) \leq \lambda \tau(\mathcal{D} \cup 0)\right\}
$$

By Proposition 4.3, and writing $K_{n} \geq c K, \forall n$, we get, for any set $\mathcal{D} \subset \mathbb{Z}^{d}$ :

$$
\begin{aligned}
& \mathbb{P}\left(N_{n}(\mathcal{D}) \geq \lambda \tau(\mathcal{D} \cup 0)\right) \\
& \leq \sum_{\left(N_{x}\right)_{x \in \mathcal{D}}, \sum_{x \in \mathcal{D}} N_{x} \geq \lambda \tau(\mathcal{D} \cup 0)} e^{-c K n} \prod_{x \in \mathcal{D}} e^{-c K N_{x}},
\end{aligned}
$$

since at least one $N_{x} \neq 0$. So, writing $e^{-c K N_{x}}=e^{-N_{x}} e^{-(c K-1) N_{x}}$, we get, for $K$ large enough (i.e. for $\epsilon$ small enough),

$$
\begin{aligned}
& \mathbb{P}\left(N_{n}(\mathcal{D}) \geq \lambda \tau(\mathcal{D} \cup 0)\right) \\
& \leq \exp (-c K n) \sum_{\left(N_{x}\right)_{x \in \mathcal{D}}} \prod_{x \in \mathcal{D}} e^{-(c K-1) N_{x}} \exp (-\lambda \tau(\mathcal{D} \cup 0)) \\
& \leq \exp (-c K n)\left(1+e^{-c K / 2}\right)^{|\mathcal{D}|} \exp (-\lambda \tau(\mathcal{D} \cup 0)) \\
& \leq \exp (-c K n) \exp (-\lambda \tau(\mathcal{D} \cup 0) / 2)
\end{aligned}
$$

Since $\sum_{\mathcal{D} \subset \mathbb{Z}^{d}} \exp (-\lambda \tau(\mathcal{D} \cup 0) / 2) \leq C, \sum_{n} \exp (-c K n) \leq C$, we get from the first Borel-Cantelli lemma, that

$$
\mathbb{P}(\bar{\Omega})=1 .
$$

Since $\|E\|_{1}$ is bounded and $E_{n}(y)=L^{n d} E\left(0, L^{n} y\right)$ we have

$$
\lim _{n \rightarrow \infty} \int d y \mathbb{1}\left(|y| \geq L^{-n / 2}\right) E_{n}(y)=0,
$$

Let $\omega \in \bar{\Omega}$. Then, for $|y| \leq L^{-n / 2}$, we get $y \in \mathbf{0}$, and, $\forall u$ and $n$ large enough,

$$
-N_{n}(\mathcal{D})+\lambda \tau(\mathcal{D} \cup u \cup 0) \geq 0 .
$$

Then, from (5.22, 4.29, 5.23) and the bounds (4.46,4.48, 5.24 5.25), we get that, for $|y| \leq L^{-n / 2}, t=0$,

$$
\int d x\left|b_{n}(x, y)\right| \leq C \epsilon_{n}^{(1-3 \gamma)}
$$

This implies

$$
\left|\int d x d y G(x) \nabla \cdot b_{n}(x, y) \mathbb{1}\left(|y| \leq L^{-n / 2}\right) E_{n}(y)\right|
$$




$$
\begin{aligned}
& \leq \int d x d y \mid \nabla \cdot G(x) \| b_{n}\left(x, y \mid \mathbb{1}\left(|y| \leq L^{-n / 2}\right) E_{n}(y)\right. \\
& \leq C \epsilon_{n}^{(1-3 \gamma)}\|\nabla \cdot G(x)\|_{\infty}
\end{aligned}
$$

For any bounded function $G$, (6.8) implies

$$
\lim _{n \rightarrow \infty} \int d x \int_{0}^{1} d \lambda \int d y G(x) T_{n}(x-y) \mathbb{1}\left(|y| \geq L^{-n / 2}\right) E_{n}(y)=0
$$

so that, using (6.2), (6.3), (6.10), (6.11),

$$
\begin{aligned}
& \lim _{n \rightarrow \infty} \int d x G(x) L^{n d} E\left(L^{2 n}, L^{n} x\right)= \\
& \lim _{n \rightarrow \infty} \int d x \int_{0}^{1} d \lambda \int d y G(x) T_{n}(x-y) \mathbb{1}\left(|y| \leq L^{-n / 2}\right) E_{n}(y) .
\end{aligned}
$$

By Proposition 4.5, we get, since $G$ is bounded,

$$
\lim _{n \rightarrow \infty} \int d x|G(x)|\left|T_{n}(x)-T_{D}^{*}(x)\right|=0 .
$$

where $D$ is defined in (6.1). One may rewrite (6.8) as

$$
\lim _{n \rightarrow \infty} \int d y \mathbb{1}\left(|y| \leq L^{-n / 2}\right) E_{n}(y)=\int d y E(y)=\|E\|_{1},
$$

and, using the bound (4.57) on $\nabla T$, we get:

$$
\int d y \mathbb{1}\left(|y| \leq L^{-n / 2}\right)\left(T_{n}(x-y)-T_{n}(x)\right) \leq C L^{-n} .
$$

Combining the last three equations, we get

$$
\lim _{n \rightarrow \infty} \int d x \int_{0}^{1} d \lambda \int d y G(x)\left(\mathbb{1}\left(|y| \leq L^{-n / 2}\right) T_{n}(x-y) E_{n}(y)-\|E\|_{1} T_{D}^{*}(x)\right)=0 .
$$

Combining this with (6.12) concludes the proof.

\section{Proof of Corollary 2.3.}

Since the equation for $E(\underline{1.6})$ is of the same form as (1.6), we need only to prove that the assumptions of the Corollary imply those of Theorem 2.1. We prove in the Appendix that there is a map $\Gamma^{*}$ conjugating the SRB measure $\nu$ for the random field $\theta$ and a Gibbs measure $\mu$ for the random field $\omega$ (see (2.2) ). We also prove in the Appendix that the Gibbs measure $\mu$ satisfies the assumptions (2.3) and (2.4) that were assumed for the random field $\omega$ in Theorem 2.1.

So, we have only to show that assumption (vi') implies the representation (2.19) and the bound (2.20) for the $b$ defined in (2.22). Since (vi') assumes (2.5) that itself implies (as is also shown in the Appendix) (2.6 ), (2.7), we can write: $b(0, x, 0, \theta)=$ $b(0, x, 0, \Gamma(\omega))=\sum_{A \subset \mathbb{Z}^{d+1}} b_{A}(x, \omega)$, with:

$$
\sum_{A \subset \mathbb{Z}^{d+1}}\left|b_{A}(x, \omega)\right| e^{\lambda d(A \cup\{0\})}<\epsilon^{\prime} e^{-m|x|},
$$


since we assumed that $C(w)=\epsilon^{\prime} e^{-m|x|}$. Now use $b(x, y, 0, \theta(t))=b\left(0, y-x, 0, \tau_{x} \theta(t)\right)$, which follow from assumption (iii') and, see (2.8), $\theta(t)=h^{t}(\theta), \tau_{x} h^{t}(\theta)=\Gamma\left(\tau_{x} \circ \tau^{t}(\omega)\right)$ to obtain: $b(x, y, 0, \theta(t))=\sum_{A \subset \mathbb{Z}^{d+1}} b_{t, A}(x, y, \omega)$, with

$$
\sum_{A \subset \mathbb{Z}^{d+1}}\left|b_{t, A}(x, y, \omega)\right| e^{\lambda d(A \cup(x, t))}<\epsilon^{\prime} e^{-m|x-y|}
$$

since, in $\tau_{x} \circ \tau^{t}(\omega)$, the origin is shifted to $(x, t)$.

Now, use the inequality

$$
d(A \cup(x, t))+|x-y| \geq d(A \cup(x, t) \cup(y, t)),
$$

to get that $b(x, y, 0, \theta(t))$ has the representation (2.19), with (2.20) holding for $\lambda \leq \frac{1}{2} m$, and $\epsilon=C \epsilon^{\prime}$.

\section{Appendix: Gibbs States}

7.1. Infinite Volume Gibbs States. We will prove in this Appendix the estimates (a) and (b) of Section 2.2., for the class of Gibbs states corresponding to SRB measures and we will explain this correspondence in the second section of this Appendix. We start by briefly recalling some definitions pertaining to Gibbs states. For a more thorough discussion, see [7] and the general discussions [26, 27, 18].

Given $X \subset \mathbb{Z}^{d+1}$, a spin configuration $\omega$ in $X$ is an element $\omega \in \Omega_{X}$. For $Y \subset X$ denote by $\omega_{Y}$ the restriction of $\omega$ to $Y$. An interaction is defined by a family $\Phi=\left\{\Phi_{X}\right\}$ of functions indexed by finite subsets $X$ of $\mathbb{Z}^{d+1}$ :

$$
\Phi_{X}: \Omega_{X} \rightarrow \mathbb{R}
$$

Our interactions are continuous invariant under the natural action of translations by $\mathbb{Z}^{d+1}$. We let $\left\|\Phi_{X}\right\|_{\infty}$ denote the sup norm of $\Phi_{X}$ (which is finite since $\Omega_{X}$ is a finite set).

Given $\Lambda \subset \mathbb{Z}^{d+1},|\Lambda|<\infty$, and a configuration $\omega^{\prime} \in \Omega_{\Lambda^{c}}$, the Hamiltonian in $\Lambda$ with boundary conditions $\omega^{\prime}$ is defined as

$$
\mathcal{H}_{\Lambda}\left(\omega \mid \omega^{\prime}\right)=-\sum_{X \cap \Lambda \neq \emptyset} \Phi_{X}\left(\omega \vee \omega^{\prime}\right)
$$

where $\omega \vee \omega^{\prime}$ is the obvious configuration in $\mathbb{Z}^{d+1}$.

We let

$$
\nu_{\Lambda}\left(\omega \mid \omega^{\prime}\right):=\exp \left(-\mathcal{H}_{\Lambda}\left(\omega \mid \omega^{\prime}\right)\right) .
$$

Then the associated (finite volume) Gibbs measure $\mu_{\Lambda}$ is the probability distribution on $\Omega_{\Lambda}$ :

$$
\mu_{\Lambda}\left(\omega \mid \omega^{\prime}\right)=Z_{\Lambda}\left(\omega^{\prime}\right)^{-1} \nu_{\Lambda}\left(\omega \mid \omega^{\prime}\right)
$$

with the partition function

$$
Z_{\Lambda}\left(\omega^{\prime}\right)=\sum_{\omega} \nu_{\Lambda}\left(\omega \mid \omega^{\prime}\right)
$$

We also need these objects for open boundary conditions, i.e. when the sum in (7.2) is restricted to $X \subset \Lambda$. Then we write $\mathcal{H}_{\Lambda}(\omega), \nu_{\Lambda}(\omega), \mu_{\Lambda}(\omega)$ and $Z_{\Lambda}$. 
We consider interactions of the following type:

$$
\Phi=\Phi^{0}+\Phi^{1}
$$

where $\Phi^{0}$ is completely analytic, in the sense of Dobrushin and Shlosman [13, 14] (see Olivieri and Picco [23, 24] for another approach, used here, to complete analyticity). In our coupled map lattices case, $\Phi^{0}$ will be a finite range interaction of a one dimensional system (which is easily seen to be completely analytic). Let the range of $\Phi^{0}$ be $r$, i.e. $\Phi_{X}^{0}=0$ if $d(X)>r$.

For $\Phi^{1}$, we will assume:

$$
\left\|\Phi^{1}\right\|=\sum_{0 \in X} e^{\lambda d(X)}\left\|\Phi_{X}^{1}\right\|_{\infty} \leq \epsilon
$$

with $\lambda>0$. For such $\Phi^{\prime}$ 's and $\epsilon=\epsilon\left(\lambda, \Phi^{0}\right)$ small enough, it is shown in [7] that the finite volume Gibbs state (7.4) has a limit, as $\Lambda \rightarrow \mathbb{Z}^{d+1}$ (in the sense of subsets ordered by inclusion) independent of $\omega^{\prime}$, which defines a measure $\mu$. We let $\mathbb{E}$ denote the expectation with respect to $\mu$ and $\mathbb{E}_{\Lambda}$ the one with respect to $\mu_{\Lambda}$ with free boundary conditions.

To prove that the conditions (2.3, 2.4) hold for $\mu$, we shall use the approach of [7] to prove:

Lemma 7.1. For $\Phi$ as in (7.6), there exists $\epsilon\left(\lambda, \Phi^{0}\right)>0$ such that for $\epsilon \leq \epsilon\left(\lambda, \Phi^{0}\right)$ in (7.7), there are constants $m>0, C<\infty$, such that the following holds:

a) For all $\Lambda, A \subset \Lambda$ and $F: \Omega_{A} \rightarrow \mathbb{R}_{+}$

$$
\exp \left(-C \epsilon|A| e^{-m R}\right) \leq \frac{\mathbb{E}(F)}{\mathbb{E}_{\Lambda}(F)} \leq \exp \left(C \epsilon|A| e^{-m R}\right)
$$

where $R=\operatorname{dist}\left(A, \Lambda^{c}\right)$.

b) If $F_{i}: \Omega_{A_{i}} \rightarrow \mathbb{R}_{+}, i=1, \ldots, k$,

$$
\mathbb{E}\left(\prod_{i=1}^{k} F_{i}\right) \leq \prod_{i=1}^{k}\left(\mathbb{E}\left(F_{i}\right) \exp \left(C \epsilon\left|A_{i}\right| e^{-m R}\right)\right)
$$

where $R=\min _{i \neq j} \operatorname{dist}\left(A_{i}, A_{j}\right)$.

c) If $F_{i}: \Omega_{A_{i}} \rightarrow \mathbb{R}, i=1,2$, and $R=\operatorname{dist}\left(A_{1}, A_{2}\right)$ then

$$
\left|\mathbb{E}\left(F_{1} F_{2}\right)-\mathbb{E}\left(F_{1}\right) \mathbb{E}\left(F_{2}\right)\right| \leq C \min \left(\left|A_{1}\right|,\left|A_{2}\right|\right)\left\|F_{1}\right\|_{\infty}\left\|F_{2}\right\|_{\infty} \exp (-m R)
$$

Proof. a) Writing $\mathbb{E}(F)=\lim _{\Lambda^{\prime} \rightarrow \mathbb{Z}^{d+1}} \mathbb{E}_{\Lambda^{\prime}}(F)$, we get:

$$
\frac{\mathbb{E}(F)}{\mathbb{E}_{\Lambda}(F)}=\lim _{\Lambda^{\prime} \rightarrow \mathbb{Z}^{d+1}} \frac{\mathbb{E}_{\Lambda^{\prime}}(F)}{\mathbb{E}_{\Lambda}(F)}=\lim _{\Lambda^{\prime} \rightarrow \mathbb{Z}^{d+1}} \frac{\sum_{\omega \in \Omega_{\Lambda}, \omega^{\prime} \in \Omega_{\Lambda^{\prime}}} F\left(\omega^{\prime}\right) \nu_{\Lambda}(\omega) \nu_{\Lambda^{\prime}}\left(\omega^{\prime}\right)}{\sum_{\omega \in \Omega_{\Lambda} \omega^{\prime} \in \Omega_{\Lambda^{\prime}}} F(\omega) \nu_{\Lambda}(\omega) \nu_{\Lambda^{\prime}}\left(\omega^{\prime}\right)}
$$

We may also assume $\Lambda \subset \Lambda^{\prime}$.

First we perform a high-temperature expansion to the $e^{-\mathcal{H}^{1}}$ part of the Hamiltonian in the numerator and the denominator of (7.11). Note that we may replace $\Phi_{X}^{1}$ by $\Phi_{X}^{1}-\inf _{\omega} \Phi_{X}^{1}(\omega)$, by adding a constant to the Hamiltonian. Thus, we may, without loss of generality, assume that

$$
\Phi_{X}^{1} \geq 0, \quad\left\|\Phi^{1}\right\| \leq 2 \epsilon .
$$


Now, write

$$
\exp \left(\sum_{X \subset \Lambda} \Phi_{X}^{1}(\omega)+\sum_{X \subset \Lambda^{\prime}} \Phi_{X}^{1}\left(\omega^{\prime}\right)\right)=\sum_{\mathcal{X} \subset \mathcal{S}\left(\Lambda^{\prime}\right)} \prod_{X \in \mathcal{X}} f_{X}\left(\omega, \omega^{\prime}\right):=\sum_{\mathcal{X} \subset \mathcal{S}\left(\Lambda^{\prime}\right)} f_{\mathcal{X}}
$$

where $\mathcal{S}\left(\Lambda^{\prime}\right)$ is the set of subsets of $\Lambda^{\prime}$, and

$$
f_{X}\left(\omega, \omega^{\prime}\right)=\exp \left(\Phi_{X}^{1}(\omega) \mathbb{1}(X \subset \Lambda)+\Phi_{X}^{1}\left(\omega^{\prime}\right)\right)-1
$$

satisfies by (7.12)

$$
0 \leq f_{X}
$$

and

$$
\sum_{0 \in X} e^{\lambda d(X)}\left\|f_{X}\right\|_{\infty} \leq C \epsilon
$$

Define, for $\mathcal{S} \subseteq \mathcal{S}\left(\Lambda^{\prime}\right)$

$$
\mathcal{N}_{\mathcal{S}}=\sum_{\mathcal{X} \subset \mathcal{S}} \sum_{\omega \in \Omega_{\Lambda}, \omega^{\prime} \in \Omega_{\Lambda^{\prime}}} F\left(\omega^{\prime}\right) f_{\mathcal{X}}\left(\omega, \omega^{\prime}\right) \exp \left(-\mathcal{H}_{\Lambda}^{0}(\omega)-\mathcal{H}_{\Lambda^{\prime}}^{0}\left(\omega^{\prime}\right)\right)
$$

and $\mathcal{D}_{\mathcal{S}}$ is the same expression with $F\left(\omega^{\prime}\right)$ replaced by $F(\omega)$. Then

$$
\frac{\mathbb{E}_{\Lambda^{\prime}}(F)}{\mathbb{E}_{\Lambda}(F)}=\frac{\mathcal{N}_{\mathcal{S}\left(\Lambda^{\prime}\right)}}{\mathcal{D}_{\mathcal{S}\left(\Lambda^{\prime}\right)}}
$$

Let now $\rho>2 r$ where $r$ is the range of $\Phi^{0}$. We cover $\mathbb{Z}^{d}$ by disjoint cubes of side $\rho$, called $\rho$-cubes. Two cubes are adjacent if their distance is less than $\rho$, and that implies the notion of connected family of $\rho$-cubes in the usual way. Given $X \subset \mathbb{Z}^{d}$ we will denote by $\underline{X}$ the set of $\rho$-cubes intersected by $X$ and by $\underline{\mathcal{X}}=\cup_{X \in \mathcal{X}} \underline{X}$. Let $\mathcal{P}=\mathcal{P}(\mathcal{X})=\left\{P_{1}, \ldots, P_{n}\right\}$ be the family of connected components of $\underline{\mathcal{X}}$ intersecting both $\underline{A}$ and $\Lambda^{c}$ and write $\mathcal{X}=\mathcal{X}_{1} \cup \mathcal{X}_{2}$ with $\mathcal{X}_{1}$ collecting the $X \subset \cup P_{i}$. We have

$$
f_{\mathcal{X}} \leq \bar{f}_{\mathcal{X}_{1}} f_{\mathcal{X}_{2}}
$$

where we set $\bar{f}_{X}:=\left\|f_{X}\right\|_{\infty}$. Given a connected union of $\rho$-cubes $P$ set

$$
\phi_{P}:=\sum_{\mathcal{X}: \underline{\mathcal{X}}=P} \bar{f}_{\mathcal{X}}
$$

With these preliminaries we may now estimate the numerator by

$$
\mathcal{N}_{\mathcal{S}\left(\Lambda^{\prime}\right)} \leq \sum_{\mathcal{P} \in \Pi_{A}} \phi_{\mathcal{P}} \mathcal{N}_{\mathcal{S}_{\mathcal{P}}}
$$

where $\Pi_{A}$ is the set of families $\mathcal{P}=\left\{P_{1}, \ldots, P_{n}\right\}$ where $P_{i}$ is a union of $\rho$-cubes, with $\operatorname{dist}\left(P_{i}, P_{j}\right) \geq \rho$ and where each $P_{i}$ intersects both $\underline{A}$ and $\Lambda^{c}$, and $\phi_{\mathcal{P}}=\prod_{i} \phi_{P_{i}}$. $\mathcal{S}_{\mathcal{P}}$ in turn consists of families $\mathcal{X} \subset \mathcal{S}\left(\Lambda^{\prime}\right)$ s.t. $\underline{\mathcal{X}}$ is disconnected from $\cup_{P \in \mathcal{P}} P$ and no connected component of $\underline{\mathcal{X}}$ intersects both $\underline{A}$ and $\Lambda^{c}$. Since $\mathcal{S}_{\mathcal{P}} \subset \mathcal{S}\left(\Lambda^{\prime}\right)$ we have $\mathcal{D}_{\mathcal{S}\left(\Lambda^{\prime}\right)} \geq \mathcal{D}_{\mathcal{S}_{\mathcal{P}}}$ and so

$$
\frac{\mathbb{E}_{\Lambda^{\prime}}(F)}{\mathbb{E}_{\Lambda}(F)} \leq \sum_{\mathcal{P} \in \Pi_{A}} \phi_{\mathcal{P}} \frac{\mathcal{N}_{\mathcal{S}_{\mathcal{P}}}}{\mathcal{D}_{\mathcal{S}_{\mathcal{P}}}}
$$

To understand our next step, let us suppose for a moment that $\mathcal{H}^{0}=0$. Then, the ratio on the RHS equals 1 . Indeed, let, for $\mathcal{X} \subset \mathcal{S}_{\mathcal{P}}, \mathcal{X}=\mathcal{X}_{A} \cup \mathcal{X}_{A^{c}}$ with $\mathcal{X}_{A}$ collecting the $X$ 
lying in connected components of $\underline{\mathcal{X}}$ that intersect $\underline{A}$ (and that are contained in $\Lambda$, by definition of $\left.\mathcal{S}_{\mathcal{P}}\right)$. Then, $\mathcal{X}_{A}, \mathcal{X}_{A^{c}}$ are disjoint and, for $X \in \mathcal{X}_{A}, f_{X}\left(\omega, \omega^{\prime}\right)=f_{X}\left(\omega^{\prime}, \omega\right)$ since $X \subset \Lambda$ (recall (7.14) $)$. Thus

$$
\sum_{\omega, \omega^{\prime}} F\left(\omega^{\prime}\right) f_{\mathcal{X}}\left(\omega, \omega^{\prime}\right)=\sum_{\omega, \omega^{\prime}} F\left(\omega^{\prime}\right) f_{\mathcal{X}_{A}}\left(\omega, \omega^{\prime}\right) \sum_{\omega, \omega^{\prime}} f_{\mathcal{X}_{A^{c}}}\left(\omega, \omega^{\prime}\right)=\sum_{\omega, \omega^{\prime}} F(\omega) f_{\mathcal{X}}\left(\omega, \omega^{\prime}\right)
$$

where in the second step we used in the first sum the above symmetry of $f$ and then interchanged $\omega$ and $\omega^{\prime}$. This renders the numerator equal to the denominator.

To be able to use this trick, we need to decouple the correlations induced by $\mathcal{H}^{0}$. Given $\mathcal{X} \subset \mathcal{S}_{\mathcal{P}}$, let

$$
V=V(\mathcal{X})=\underline{\mathcal{X}} \cup \underline{A}
$$

Then $F f_{\mathcal{X}}$ depends only on $\omega_{V \cap \Lambda}, \omega_{V}^{\prime}$ and summing over the other variables we get

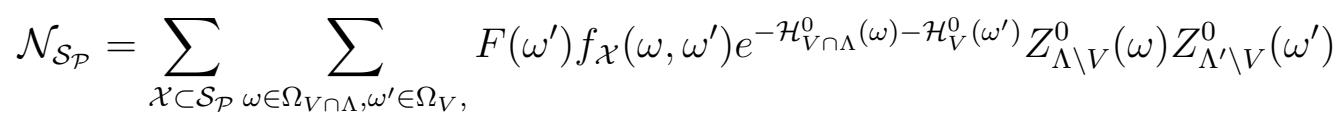

where, with a slight abuse of notation, $Z_{\Lambda \backslash V}^{0}(\omega)$ is the partition function with interaction $\Phi^{0}, \omega$ boundary condition in $V \cap \Lambda$, and open boundary conditions in $\Lambda^{c}$ and similarly for $Z_{\Lambda^{\prime} \backslash V}^{0}\left(\omega^{\prime}\right)$. $\mathcal{D}_{\mathcal{S}_{\mathcal{P}}}$ has an analogous representation.

Observe that, since the range of $\Phi^{0}$ is less than $\rho / 2$, the exponential in (17.24) factors over the connected components of $V_{\alpha}$ of $V$ :

$$
\zeta_{V}\left(\omega, \omega^{\prime}\right):=\exp \left(-\mathcal{H}_{V \cap \Lambda}^{0}(\omega)-\mathcal{H}_{V}^{0}\left(\omega^{\prime}\right)\right)=\prod_{\alpha} \zeta_{V_{\alpha}}\left(\omega, \omega^{\prime}\right)
$$

where $\zeta_{V_{\alpha}}\left(\omega, \omega^{\prime}\right)$ is a function of $\omega_{V_{\alpha} \cap \Lambda}, \omega_{V_{\alpha}}^{\prime}$. However, the partition functions do not factor and we need to expand them. For this, we will use the

Lemma 7.2. There exists a constant $z_{\Lambda^{\prime} \backslash V}$ and functions $W_{A}$ on $\Omega_{A}$ and $\phi_{Y}$ on $\Omega_{Y \cap V}$ such that

$$
Z_{\Lambda^{\prime} \backslash V}^{0}\left(\omega^{\prime}\right)=z_{\Lambda^{\prime} \backslash V} \prod_{\alpha} W_{V_{\alpha}}\left(\omega^{\prime}\right) \exp \sum_{Y \in \mathcal{U}_{V}} \phi_{Y}\left(\omega^{\prime}\right)
$$

where $\mathcal{U}_{V}$ is the set of connected sets of $\rho$-cubes $Y \subset \Lambda^{\prime}$, so that $Y \cap V \neq \emptyset$ and $Y \cap V^{c} \neq \emptyset$. Moreover, for any $\epsilon>0$, there exist $\rho<\infty, \lambda>0$ such that

$$
\sum_{0 \in Y} \exp \left(\frac{\lambda d(Y)}{\rho}\right)\left\|\phi_{Y}\right\|_{\infty} \leq \epsilon
$$

A similar representation holds for $Z_{\Lambda \backslash V}^{0}(\omega)$.

Proof. The Lemma goes back to Olivieri and Picco [23, 24] and is discussed in [7], see formula (13) (note that there we had $\phi_{Y}$ constant if $Y \cap V=\emptyset$, so that here $\left.z_{\Lambda^{\prime} \backslash V}=\exp \left(f\left|\Lambda^{\prime} \backslash V\right|+\sum_{Y \subset \Lambda^{\prime} \backslash V} \phi_{Y}\right)\right)$. Note also that there is no loss of generality in assuming that $\lambda$ here is the same as in (7.16).

Define now

$$
\bar{\phi}_{Y}=\min _{\omega} \phi_{Y}(\omega)
$$

and

$$
\Psi_{Y}(\omega):=\phi_{Y}(\omega)-\bar{\phi}_{Y}
$$


whereby we have

$$
\Psi_{Y} \geq 0 \text {. }
$$

We will now apply (7.26) to each of the partition functions in eq. (7.24) and the corresponding expression for the denominator $\mathcal{D}_{\mathcal{S}_{\mathcal{P}}}$. We get:

$$
e^{-\mathcal{H}_{V \cap \Lambda}^{0}(\omega)-\mathcal{H}_{V}^{0}\left(\omega^{\prime}\right)} Z_{\Lambda \backslash V}^{0}(\omega) Z_{\Lambda^{\prime} \backslash V}^{0}\left(\omega^{\prime}\right)=\mathcal{W}_{V} \prod_{Y \in \mathcal{U}_{V}}\left(1+g_{Y}\right)
$$

where

$$
\mathcal{W}_{V}=z_{\Lambda \backslash V} z_{\Lambda^{\prime} \backslash V} \prod_{Y \in \mathcal{U}_{V}} e^{\left.\bar{\phi}_{Y}(\mathbb{1}(Y \subset \Lambda)+1)\right)} \prod_{\alpha} W_{V_{\alpha} \cap \Lambda}(\omega) W_{V_{\alpha}}\left(\omega^{\prime}\right) \zeta_{V_{\alpha}}\left(\omega, \omega^{\prime}\right)
$$

We also denoted

$$
g_{Y}=\exp \left(\Psi_{Y}(\omega) \mathbb{1}(Y \subset \Lambda)+\Psi_{Y}\left(\omega^{\prime}\right)\right)-1
$$

By (7.30)

$$
0 \leq g_{Y}
$$

and by (7.27)

$$
\sum_{0 \in Y} \exp \left(\frac{\lambda d(Y)}{L}\right)\left\|g_{Y}\right\|_{\infty} \leq C \epsilon .
$$

If we use (7.31), (7.32), we see that the obstruction to the factorization, as in (7.22), of the sums in (17.24), comes only from the product of $1+g_{Y}$. Thus, we expand:

$$
\prod_{Y \in \mathcal{U}_{V}}\left(1+g_{Y}\right)=\sum_{\mathcal{Y} \subset \mathcal{S}\left(\mathcal{U}_{V}\right)} \prod_{Y \in \mathcal{Y}} g_{Y}:=\sum_{\mathcal{Y} \subset \mathcal{S}\left(\mathcal{U}_{V}\right)} g_{\mathcal{Y}}
$$

where $\mathcal{S}\left(\mathcal{U}_{V}\right)$ is the set of subsets of $\mathcal{U}_{V}$.

Combining (7.24), (7.31) and (7.36) we get

$$
\mathcal{N}_{\mathcal{S}_{\mathcal{P}}}=\sum_{\mathcal{X} \subset \mathcal{S}_{\mathcal{P}}} \sum_{\mathcal{Y} \subset \mathcal{S}\left(\mathcal{U}_{V}\right)} \sum_{\omega \in \Omega_{V \cap \Lambda}, \omega^{\prime} \in \Omega_{V}} F\left(\omega^{\prime}\right) f_{\mathcal{X}} g_{\mathcal{Y}} \mathcal{W}_{V}
$$

We repeat now what we did with $\mathcal{X}$. Let $\mathcal{Q}=\mathcal{Q}(\mathcal{X}, \mathcal{Y})=\left\{Q_{1}, \ldots, Q_{m}\right\}$ be the family of connected components of $\underline{\mathcal{X}} \cup \underline{\mathcal{Y}}$ intersecting both $\underline{A}$ and $\Lambda^{c}$ and write $\mathcal{Y}=\mathcal{Y}_{A} \cup \mathcal{Y}_{A^{c}}$ with $\mathcal{Y}_{A}$ collecting the $Y \subset \cup Q_{i}$ and similarly for $\mathcal{X}$. We have again

$$
f_{\mathcal{X}} g_{\mathcal{Y}} \leq \bar{f}_{\mathcal{X}_{A}} f_{\mathcal{X}_{A^{c}}} \bar{g}_{\mathcal{Y}_{A}} g_{\mathcal{Y}_{A^{c}}}
$$

Given a union of $\rho$-cubes $Q$, set

$$
\psi_{Q}^{\mathcal{P}}:=\sum_{\mathcal{X} \subset \mathcal{S}_{\mathcal{P}}, \mathcal{Y} \subset \mathcal{S}\left(\mathcal{U}_{V}\right)} \bar{f}_{\mathcal{X}} \bar{g}_{\mathcal{Y}} 1_{\mathcal{Q}(\mathcal{X}, \mathcal{Y})=\{Q\}} .
$$

We get

$$
\mathcal{N}_{\mathcal{S}_{\mathcal{P}}} \leq \sum_{\mathcal{Q} \in \Pi_{A}} \psi_{\mathcal{Q}}^{\mathcal{P}} \mathcal{N}_{\mathcal{S}_{\mathcal{P}, \mathcal{Q}}}
$$

with $\psi_{\mathcal{Q}}^{\mathcal{P}}=\prod_{Q \in \mathcal{Q}} \psi_{Q}^{\mathcal{P}}$, and

$$
\mathcal{N}_{\mathcal{S}_{\mathcal{P}, \mathcal{Q}}}=\sum_{\{\mathcal{X}, \mathcal{Y}\} \in \mathcal{S}_{\mathcal{P}, \mathcal{Q}}} \sum_{\omega \in \Omega_{V \cap \Lambda}, \omega^{\prime} \in \Omega_{V}} F\left(\omega^{\prime}\right) f_{\mathcal{X}} g_{\mathcal{Y}} \mathcal{W}_{V}
$$


where $\mathcal{S}_{\mathcal{P}, \mathcal{Q}}$ consist of pairs $(\mathcal{X}, \mathcal{Y}), \mathcal{X} \subset \mathcal{S}_{\mathcal{P}}, \mathcal{Y} \subset \mathcal{S}\left(\mathcal{U}_{V}\right)$ such that $\underline{\mathcal{X}} \cup \underline{\mathcal{Y}}$ is disconnected from $\cup_{Q \in \mathcal{Q}} Q$ and no connected component of $\underline{\mathcal{X}} \cup \underline{\mathcal{Y}}$ intersects both $\underline{A}$ and $\Lambda^{c}$.

There is a similar expansion in the denominator and, since the $\mathcal{X}$ part of $\mathcal{S}_{\mathcal{P}, \mathcal{Q}}$ belongs to $\mathcal{S}_{\mathcal{P}}$, we have $\mathcal{D}_{\mathcal{S}_{\mathcal{P}}} \geq \mathcal{D}_{\mathcal{S}_{\mathcal{P}, \mathcal{Q}}}$ and then

$$
\frac{\mathbb{E}_{\Lambda^{\prime}}(F)}{\mathbb{E}_{\Lambda}(F)} \leq \sum_{\mathcal{P} \in \Pi_{A}} \sum_{\mathcal{Q} \in \Pi_{A}} \phi_{\mathcal{P}} \psi_{\mathcal{Q}}^{\mathcal{P}} \frac{\mathcal{N}_{\mathcal{S}_{\mathcal{P}, \mathcal{Q}}}}{\mathcal{D}_{\mathcal{S}_{\mathcal{P}, \mathcal{Q}}}}=\sum_{\mathcal{P} \in \Pi_{A}} \sum_{\mathcal{Q} \in \Pi_{A}} \phi_{\mathcal{P}} \psi_{\mathcal{Q}}^{\mathcal{P}}
$$

where in the last step we applied the symmetry argument as in the special case (7.22) above, which holds here by definition of $\mathcal{S}_{\mathcal{P}, \mathcal{Q}}$.

Let us bound $\phi_{P}$ in (7.19). Let $\underline{\mathcal{X}}=P$ with $\mathcal{X}=\left\{X_{1}, \ldots, X_{n}\right\}$. Then $d(P) \leq$ $\sum_{i} d\left(X_{i}\right)+2 n \rho$. Let $\mathcal{A}$ be the the set of connected $P$ s.t. $P$ intersects both $\underline{A}$ and $\Lambda^{c}$. Then,

$$
\sum_{P \in \mathcal{A}} \phi_{P} \leq e^{-\frac{1}{2} \lambda d\left(A, \Lambda^{c}\right)} \sum_{\mathcal{X}: \underline{\mathcal{X}} \in \mathcal{A}} \prod_{i} e^{\frac{1}{2} \lambda d\left(X_{i}\right)+2 \rho} \bar{f}_{X_{i}} .
$$

Using (7.16) the sum is readily bounded, since $P$ is connected and must contain a point in $A$, by $C(\rho) \epsilon|A|$ and

$$
\sum_{P \in \mathcal{A}} \phi_{P} \leq C(\rho) \epsilon|A| e^{-\frac{1}{2} \lambda d\left(A, \Lambda^{c}\right)}
$$

Similar estimate holds for $\psi_{Q}^{\mathcal{P}}$, uniformly in $\mathcal{P}$, using (7.35). Therefore, since $\rho$ is only constrained to be larger that $2 r$, and $r$ is given, we can write $C(\rho)=C$, and get

$$
\text { (7.40) } \leq\left(\sum_{k=0}^{\infty} k !^{-1}\left(C \epsilon|A| e^{-\frac{1}{2} \lambda d\left(A, \Lambda^{c}\right)}\right)^{k}\right)^{2} \leq \exp \left(C \epsilon|A| e^{-\frac{1}{2} \lambda R}\right) .
$$

since $d\left(A, \Lambda^{c}\right) \geq R$. This is the upper bound in (7.8).

For the lower bound, we simply apply the argument for the upper bound to the ratio $\mathbb{E}_{\Lambda}(F) / \mathbb{E}_{\Lambda^{\prime}}(F)$.

To prove (17.9) let $\Lambda_{i}=\left\{z \mid \operatorname{dist}\left(z, A_{i}\right) \leq \frac{R}{4}\right\}$, and let $\Lambda=\bigcup_{i=1}^{k} \Lambda_{i}$.

Then, $\Lambda_{i} \cap \Lambda_{j}=\emptyset$ for $i \neq j$ and

$$
\mathbb{E}_{\Lambda}\left(\prod_{i=1}^{k} F_{i}\right)=\prod_{i=1}^{k} E_{\Lambda_{i}}\left(F_{i}\right)
$$

Then using (7.8) for $F=\prod_{i=1}^{k} F_{i}$, with $A=\cup_{i=1}^{k} A_{i}$, and using

$$
\frac{\mathbb{E}_{\Lambda_{i}}\left(F_{i}\right)}{\mathbb{E}\left(F_{i}\right)} \leq \exp \left(C \epsilon\left|A_{i}\right| e^{-c R}\right)
$$

which follows from (7.8), for each $\frac{\mathbb{E}_{\Lambda_{i}}\left(F_{i}\right)}{\mathbb{E}\left(F_{i}\right)}$, we get

$$
\mathbb{E}\left(\prod_{i=1}^{k} F_{i}\right) \leq \prod_{i=1}^{k}\left(\mathbb{E}\left(F_{i}\right) \exp \left(C \epsilon\left|A_{i}\right| e^{-c R}\right)\right)
$$

i.e. (7.9)).

c) This is proven in [7]. 
7.2. Coupled map lattices. We will now recall briefly how the SRB measure of our CML fits into the framework of the previous section. For definiteness fix $N=\mathbb{T}^{2}$. Let $\mathcal{N}=N^{\mathbb{Z}^{d}}$ which is a compact metric space with the metric

$$
d\left(\theta, \theta^{\prime}\right)=\sum_{x \in \mathbb{Z}^{d}} 2^{-|x|} \rho\left(\theta(x), \theta^{\prime}(x)\right)
$$

where $\rho$ is the standard metric on $\mathbb{T}^{2}$.

Let $h: \mathcal{N} \rightarrow \mathcal{N}$ in (1.7) be of the form

$$
h(x, \theta)=A \theta(x)+k(x, \theta)
$$

where $A: \mathbb{T}^{2} \rightarrow \mathbb{T}^{2}$ is a linear hyperbolic torus automorphism and $k$ satisfies the bound (2.1) (we describe $\mathbb{T}^{2}$ here by $\theta \in[0,1]^{2}, A$ is in $S L(2, \mathbb{Z})$ and $k$ is periodic over $\left.\mathbb{Z}^{2}\right)$. Let us recall that $\left(A, \mathbb{T}^{2}\right)$ is conjugate to a subshift of finite type $(\tau, \Sigma)$ where $\Sigma \subset\{1,2, \ldots, p\}^{\mathbb{Z}}$ consists of sequences $\omega$ with $M_{\omega(t), \omega(t+1)}=1$ for all $t$ where $M$ is a $p \times p$ matrix with entries in the set $\{0,1\}$.

The construction of the SRB measure goes through finite volume approximations. Let $V_{R}$ denote the box of side $2 R+1$ in $\mathbb{Z}^{d}$ with opposite faces identified and set $\mathcal{N}_{R}=N^{V_{R}}$. Let $h_{R}: \mathcal{N}_{R} \rightarrow \mathcal{N}_{R}$ denote the periodization of $h$ i.e. given a $\theta \in \mathcal{N}_{R}$ let $\theta_{R} \in \mathcal{N}$ be its periodic extension and set $h_{R}(x, \theta)=h\left(x, \theta_{R}\right)$. For $\theta \in \mathcal{N}_{R}$ let $W_{R}(\theta)$ be the leaf of the unstable foliation passing through $\theta$ (which exists for $\kappa$ in (2.1) small enough) and $\tilde{h}_{R}: W_{R}(\theta) \rightarrow W_{R}(\theta)$ the restriction of $h_{R}$ to $W_{R}(\theta)$. Define the function

$$
\Lambda_{R}(\theta)=\log \operatorname{det} D \tilde{h}_{R}(\theta) .
$$

Using translation invariance one shows

$$
\Lambda_{R}(\theta)=\sum_{x \in V_{R}} \lambda_{R}\left(\tau_{x} \theta\right)
$$

where $\tau_{x}$ is the translation by $x$ and where the functions $\lambda_{R}$ (extended periodically to $\mathbb{Z}^{d}$ ) are uniformly Hölder continuous with

$$
\left|\lambda_{R}(\theta)-\lambda_{R}\left(\theta^{\prime}\right)\right| \leq \eta d\left(\theta, \theta^{\prime}\right)^{\alpha}
$$

where $\alpha>0$ and $\eta \rightarrow 0$ as $\kappa \rightarrow 0$ in (2.1). One has a good control of their $R$ dependence and, as $R \rightarrow \infty$, they converge to $\lambda: \mathcal{N} \rightarrow \mathcal{N}$ satisfying (7.42).

Next, let $\Sigma_{R}=\Sigma^{V_{R}}$ and $\Sigma_{\infty}=\Sigma^{\mathbb{Z}^{d}}$. Equip these with the metric

$$
d\left(\omega, \omega^{\prime}\right)=\sum_{(t, x) \in \mathbb{Z}^{d+1}} 2^{-|t|-|x|}\left|\omega(t, x)-\omega^{\prime}(t, x)\right|
$$

The map $h_{R}$ is conjugate to the time shift $\tau: \Sigma_{R} \rightarrow \Sigma_{R}$ with $\tau \omega(t, x)=\omega(t+1, x)$ i.e. there exists a Hölder continuous map $\Gamma_{R}: \Sigma_{R} \rightarrow \mathcal{N}_{R}$ with

$$
d\left(\Gamma_{R}(\omega), \Gamma_{R}\left(\omega^{\prime}\right)\right) \leq C d\left(\omega, \omega^{\prime}\right)^{\beta}
$$

for some $\beta>0$ uniformly in $R$ and

$$
h_{R}=\Gamma_{R}^{-1} \circ \tau \circ \Gamma_{R}
$$

We can now define a Hamiltonian

$$
H_{T, R}(\omega)=\sum_{t=-T}^{T} \sum_{x \in V_{R}}\left(\lambda_{R} \circ \Gamma_{R}\right)\left(\tau^{t} \tau_{x} \omega\right),
$$


and the probability measure

$$
\mu_{T, R}(d \omega)=Z_{T, R}^{-1} e^{-H_{T, R}(\omega)} \mu^{0}(d \omega)
$$

where $\mu^{0}$ is the maximum entropy measure on $\Sigma_{R}$.

Let $m_{R}$ denote the normalized Lebesgue measure on $\mathcal{N}_{R}$. One then has, see [3],

Proposition 7.3. The weak limits

$$
\lim _{n \rightarrow \infty} h_{R}^{n} m_{R}=\nu_{R} \text { and } \lim _{T \rightarrow \infty} \mu_{T, R}=\mu_{R}
$$

exist and are related by

$$
\nu_{R}=\Gamma_{R}^{*} \mu_{R}
$$

In particular $\nu_{R}$ is the SRB measure of the dynamical system $\left(h_{R}, \mathcal{N}_{R}\right)$.

We will now realize $\mu_{R}$ as a Gibbs measure with a potential satisfying the conditions of Section 7.1. We will use telescoping sums to express the function $\ell_{R}:=\lambda_{R} \circ \Gamma_{R}$ as a sum of localized functions. Let us order the set $\mathbb{Z}^{d+1}=\left\{z_{1}, z_{2}, \ldots\right\}$ s.t. $\left|z_{i+1}\right| \geq\left|z_{i}\right|$. Given $\omega \in \Sigma_{R}$, let $\omega_{n}\left(z_{i}\right)=\omega\left(z_{i}\right)$ if $i \leq n$ and $\omega_{n}\left(z_{i}\right)=\tilde{\omega}\left(z_{i}\right)$ where for each $n$ we choose an arbitrary extension $\tilde{\omega}$ of $\Omega_{n}:=\left\{\omega\left(z_{i}\right) \mid i \leq n\right\}$ to $\Sigma_{R}$ (it is not hard to see that for each $\Omega_{n}$ such an extension can be chosen). We can then write

$$
\ell_{R}(\omega)=\ell_{R}\left(\omega_{1}\right)+\sum_{n=1}^{\infty}\left(\ell_{R}\left(\omega_{n+1}\right)-\ell_{R}\left(\omega_{n}\right)\right):=\sum_{n=0}^{\infty} \phi_{R, A_{n}}(\omega),
$$

where $\phi_{R, A_{n}}$ is localized in a region $A_{n}$ with $d\left(A_{n}\right)$ comparable to $(1+n)^{1 / d}$. Moreover, since $\omega_{n}$ and $\omega_{n+1}$ agree in a space time region of radius comparable to $d\left(A_{n}\right)$ we get from (7.42) and (7.43),

Given $A \subset \mathbb{Z}^{d+1}$ let now

$$
\left|\phi_{R, A_{n}}(\omega)\right| \leq C \eta 2^{-\alpha \beta d\left(A_{n}\right)}
$$

$$
\Phi_{R, A}(\omega)=\sum_{t, x} \phi_{R, A_{n}}\left(\tau^{t} \tau_{x} \omega\right) \mathbb{1}_{A_{n}=\tau^{t} \tau_{x} A}
$$

Since the number of sets $A_{n}$ with radius between $r$ and $r+1$ is bounded by $C r^{d}$ one easily concludes that

$$
\sum_{A: 0 \in A}\left\|\Phi_{R, A}(\omega)\right\|_{\infty} e^{\lambda d(A)} \leq C \eta
$$

for some $\lambda>0$, uniformly in $R$. We are hence in the framework of Section 7.1. and conclude that $\mu_{R}$ has a weak limit $\mu$ which is the unique Gibbs measure of the potential $\lim _{R \rightarrow \infty} \Phi_{R, .}$ In particular the assumptions (a) and (b) in section 2.2 hold for $\mu$. As a consequence $\nu_{R}$ also has a weak limit $\nu$, and it is the SRB measure of $(h, \mathcal{N})$. These measures are related by the conjugation $\nu=\Gamma^{*} \mu$.

Finally, consider a local function $w(\theta)$ as in (2.5). By the above telescoping argument (7.44) we obtain the claims (2.6) and (2.7).

If our original system is a perturbation of uncoupled expanding circle maps, i.e. $N=S^{1}$, the Jacobian of the map restricted to the unstable manifold, which is used here, is then replaced by the Jacobian of the original map. This time the subshift is replaced by the full shift on $\{1, \ldots, p\}^{\mathbb{N}}$ and the space time symbolic representation is on $\{1, \ldots, p\}^{\mathbb{Z}^{d+1}}$. 


\section{ACKNOWLEDGMENTS}

This work is partially funded by the Academy of Finland the European Research Council and the Belgian Interuniversity Attraction Pole, P6/02.

\section{REFERENCES}

[1] F. Bonetto, P. Falco, and G. Giuliani, Analyticity of the SRB measure of a lattice of coupled Anosov diffeomorphisms of the torus, Journal of Mathematical Physics 45, 3282-3309 (2004).

[2] F. Bonetto, G. Gallavotti, and G. Gentile, A fluctuation theorem in a random environment, Ergodic theory and dynamical systems $\mathbf{2 8}$, no 1 21-47 (2008).

[3] F.Bonetto, A. Kupiainen, J.L. Lebowitz Absolute continuity of projected SRB measures of coupled Arnold cat map lattices, Ergodic Theory and Dynamical Systems, 25, 59-88, (2005) (also: nlin.CD/0310009).

[4] R. Bowen, Equilibrium states and the ergodic theory of Anosov diffeomorphisms, Lecture Notes in Mathematics, 470, Springer, New York (1975).

[5] J.Bricmont, A. Kupiainen, Random Walks in Asymmetric Random Environments, Comm. Math. Phys. 142, 345-420 (1991).

[6] J.Bricmont, A. Kupiainen, Coupled Analytic Maps, Nonlinearity 8, 379-393 (1995).

[7] J.Bricmont, A. Kupiainen, High Temperature Expansion and Dynamical Systems, Commun.Math. Phys. 178, 703-732 (1996).

[8] J.Bricmont, A. Kupiainen, On the derivation of Fourier's law for coupled anharmonic oscillators, Commun.Math. Phys. 274, 555-626 (2007).

[9] J.Bricmont, A. Kupiainen, Random walks in space time mixing environments, J.Stat.Phys. 134, 979-1004 (2009).

[10] J.Bricmont, A. Kupiainen, in preparation.

[11] L.A. Bunimovich, Y.G. Sinai, Space-time chaos in coupled map lattices, Nonlinearity, 1, 491-516 (1988).

[12] L. Bunimovich, C. Liverani, A. Pellegrinotti and Yu. Suhov, Ergodic systems of $n$ balls in a billiard table, Commun.Math. Phys. 146, 357 (1992).

[13] R.L. Dobrushin, S.B. Shlosman, Completely analytical Gibbs fields, in Statistical Physics and Dynamical Systems (Rigorous Results), 371-403, Birkäuser, Boston (1985).

[14] R.L. Dobrushin, S.B. Shlosman, Completely analytical interactions: a constructive description, J. Stat. Phys. 46, 983-1014(1987).

[15] D. Dolgopyat, G. Keller, C. Liverani, Random Walk in Markovian Environment, Annals of Probability, 36, Number 5, 1676-1710 (2008).

[16] D. Dolgopyat, C. Liverani, Random Walk in Deterministically Changing Environment, ALEA, 4, 89-116 (2008).

[17] J.-P. Eckmann and L.-S. Young. Temperature profiles in Hamiltonian heat conduction. Europhysics Letters 68 (2004), 790-796.

[18] A. C. D. van Enter, R. Fernández, A. D. Sokal: Regularity properties and pathologies of positionspace renormalization-group transformations: Scope and limitations of Gibbsian theory J. Stat. Phys., 72, 879-1167 (1993).

[19] P. Gaspard, T. Gilbert, Heat conduction and Fourier's law in a class of many particle dispersing billiards, New Journal of Physics, 10, 103004 (2008).

[20] M. Jiang, Sinai-Ruelle-Bowen measures for lattice dynamical systems Journal of statistical physics 111, 863 - 902 (2003).

[21] M. Jiang, Y. B. Pesin, Equilibrium measures for coupled map lattices: Existence, uniqueness and finite dimensional approximations, Communications in mathematical physics, 193, 675-711 (1998).

[22] J. Lukkarinen, H. Spohn, Weakly nonlinear SchrËĘdinger equation with random initial data, arXiv:0901.3283v1 [math-ph].

[23] E. Olivieri, On a cluster expansion for lattice spin systems: a finite-size condition for the convergence, J. Stat. Phys., 50, 1179-1200 (1988). 
[24] E. Olivieri, P. Picco, Cluster expansion for d-dimensional lattice systems and finite volume factorization properties, J. Stat. Phys., 59, 221-256 (1990).

[25] Y.G. Pesin, Y.G. Sinai, Space-time chaos in chains of weakly coupled hyperbolic maps, in: Advances in Soviet Mathematics, Vol. 3, ed. Y.G. Sinai , Harwood (1991).

[26] D. Ruelle, Thermodynamic Formalism, Addison-Wesley (1978).

[27] B. Simon, The Statistical Mechanics of Lattice Gases, Vol. 1, Princeton Univ. Press (1994).

[28] Y.G. Sinai, Gibbs measures in ergodic theory, Russian Math. Surveys 27, 21-64 (1972).

UClouvain, IRMP, Chemin du Cyclotron 2, B-1348 Louvain-la-Neuve, Belgium

E-mail address: Jean.Bricmont@uclouvain.be

University of Helsinki, Department of Mathematics and Statistics, P.O. Box 68, FIN-00014 University of Helsinki, Finland

E-mail address: antti.kupiainen@helsinki.fi 